\title{
Structural basis for regulation of a tripartite toxin-antitoxin system by dual phosphorylation
}

René L. Boerentsen ${ }^{1 *}$, Stine Vang Nielsen ${ }^{2 *}$,Jeppe Lyngsø ${ }^{3}$, Francesco Bisiak $^{1}$, Jan Skov Pedersen ${ }^{3}$, Kenn Gerdes ${ }^{4}$, Michael A. Sørensen ${ }^{2 \dagger}$, Ditlev. E. Brodersen ${ }^{1 \dagger}$

${ }^{1}$ Department of Molecular Biology and Genetics, Aarhus University, Gustav Wieds Vej 10c, DK8000 Aarhus C, Denmark, ${ }^{2}$ Department of Biology, University of Copenhagen, Ole Maaløes Vej 5, DK-2200 København N, ${ }^{3}$ Department of Chemistry and Interdisciplinary Nanoscience Centre (iNANO), Gustav Wieds Vej 14, DK-8000 Aarhus C, ${ }^{4}$ Voldmestergade 8, DK-2100 København Ø, Denmark.

${ }^{*}$ These authors contributed equally.

†To whom correspondence should be addressed (mas@bio.ku.dk, phone +45 35323711 or deb@mbg.au.dk, phone+45 21669001).

Keywords: HipBA; STK; Ser/Thr kinase; TrpS; tRNA synthetase

Running title: Regulation of a tripartite TA system 


\section{ABSTRACT}

2 Many bacteria encode multiple toxin-antitoxin (TA) systems targeting separate, but closely related,

3 cellular functions. The toxin of the E. coli hipBA system, HipA, is a kinase that inhibits inhibits

4 translation via phosphorylation of glutamyl-tRNA synthetase. Enteropathogenic E. coli (EPEC)

5 O127:H6 encodes an additional, tripartite TA module, hipBST, for which the HipT toxin was shown

6 to specifically target tryptophanyl-tRNA synthetase, TrpS. Surprisingly, the function as antitoxin has

7 been taken over by the third protein, HipS, but the molecular details of how activity of HipT is

8 controlled remain poorly understood. Here, we show that HipBST is markedly different from HipBA

9 and that the unique HipS protein, which is homologous to the N-terminal subdomain of HipA, has

10 evolved to function as antitoxin by breaking the kinase active site. We also show how auto-

11 phosphorylation at two conserved sites in the kinase toxin serve to dually regulate binding of HipS

12 and kinase activity. Finally, we demonstrate that the HipBST complex is dynamic and present a

13 cohesive model for the regulation and activation of this type of three-component system. 


\section{INTRODUCTION}

Bacteria employ a wide range of mechanisms adapt to changing environments and imminent threats such as antibiotics and phage attack, including activation of toxin-antitoxin (TA) systems that can release intracellular toxins to rapidly alter metabolism or reduce growth (Harms et al., 2018). Canonical (type II) TA systems are small, bicistronic loci that encode a protein toxin and its cognate antidote (antitoxin) that tightly interact to form a higher-order complex in the inactive state, usually capable of controlling transcription via a DNA-binding domain on the antitoxin (Harms et al., 2018). There are also examples of tricistronic TA loci, in which the third component is either a chaperone required for folding of the antitoxin and thus, toxin inhibition (Bordes et al., 2016) or an additional, transcriptional regulator (Hallez et al., 2010; Zielenkiewicz \& Ceglowski, 2005). A striking observation is that some microorganisms contain a very large number of similar, paralogous TA systems, such as the human pathogen, Mycobacterium tuberculosis, which contains at least 47 paralogous TA systems of the vapBC-type, for which the VapC toxin appears to target unique noncoding RNA species (Sharrock et al., 2018; Winther et al., 2016). Likewise, Salmonella enterica encodes several paralogous tacTA systems in which the TacT toxins function by acetylating the primary amine of amino acids of charged transfer RNA molecules (Grabe et al., 2021). These cases raise important questions about the evolutionary benefit of harbouring multiple, highly similar toxin systems as well as how cross-reactivity is prevented.

In the widespread and diverse hipBA (high persister) system, the HipA toxin is a serine-threonine kinase (STKs) (Hanks et al., 1988; Stancik et al., 2018), while the cognate antitoxin, HipB, contains a helix-turn-helix (HTH) motif and inhibits the toxin by a mechanism which is not fully understood (Gerdes et al., 2021; Schumacher et al., 2009). In the most well-studied and canonical hipBA system in Escherichia coli K-12, the toxin (HipA $\mathrm{Ec}_{\mathrm{C}}$ ) specifically targets glutamyl-tRNA synthetase (GltX) 
by phosphorylation of an ATP-binding motif conserved in type I aminoacyl-tRNA synthetases (Eriani et al., 1990; Sekine et al., 2003), thereby inhibiting its activity and blocking translation (Germain et al., 2013). Accumulation of uncharged tRNA ${ }^{\text {Glt }}$ subsequently induces the stringent response via RelAmediated (p)ppGpp synthesis on starved ribosomes (Haseltine \& Block, 1973; Pacios et al., 2020; Winther et al., 2018). In contrast to most type II TA systems, the cognate antitoxin, HipB does not directly interact with the HipA active site and inhibition has therefore been proposed to occur by several mechanisms, including blocking conformational changes in the kinase required for catalysis, sequestration of HipA on DNA via the HipB DNA binding domain (Schumacher et al., 2009), or allosterically via placement of a C-terminal Trp residue of HipB into a pocket on HipA (Evdokimov et al., 2009). In an additional layer of complexity in regulation, $\mathrm{Hip}_{\mathrm{Ec}}$ is regulated by trans autophosphorylation at a conserved serine (Ser150) situated in a loop (the "Gly-rich loop") near the active site, which is required for ATP binding and functionally similar to the P loop in eukaryotic kinases (Huse \& Kuriyan, 2002; Schumacher et al., 2012). Phosphorylation at this site causes a conformational change in which the loop is ejected from its burrowed position inside the active site, rendering the kinase unable to bind ATP (Schumacher et al., 2012).

Enteropathogenic E. coli (EPEC) O127:H6, an important diarrheal pathogen of young children, contains, in addition to the canonical hipBA locus, a homologous tricistronic TA system, hipBST, encoding three separate proteins. HipB, by analogy to HipBA, contains a putative DNA-binding HTH domain while HipT is a kinase like HipA and functions as toxin by phosphorylating and inactivating tryptophanyl-tRNA, TrpS (Gerdes et al., 2021; Vang Nielsen et al., 2019). Surprisingly, the third protein, HipS, which at the sequence level corresponds to the $\mathrm{N}$-terminal subdomain 1 of the larger HipA kinase, was shown to function as antitoxin by an unknown mechanism (Vang Nielsen et al., 2019). Moreover, HipT was shown to be subject to autophosphorylation at two conserved serine 
positions in the Gly-rich loop, but the functional consequences of this are also not known. Here, we show that the HipBST complex fom pathogenic E. coli is markedly different from HipBA and that inhibition of HipT is achieved through direct interaction with a conserved residue in HipS. Two structures of HipT in separate, phosphorylated states further demonstrate that HipT, is found in an inactive conformation regardless of phosphorylation state inside the HipBST complex. Finally, we show, using phosphomimetic kinase variants in vivo and analysis of phosphorylation patterns and flexibility in vitro, that the two phosphorylation positions control HipT activity and binding of antitoxin (HipS), and that the HipBST complex is dynamic. Together, our data provide a mechanistic understanding of how the HipS protein can function as antitoxin and suggests that the corresponding domain in HipA can have important, regulatory roles as well.

\section{RESULTS}

\section{Dual autophosphorylation sites are conserved in HipT kinases}

One of the most surprising features of the HipBST system is that HipS shows sequence similarity to the N-terminal subdomain 1 of HipA, the toxin of the hipBA system, while simultaneously functioning as antitoxin (Figure S1) (Vang Nielsen et al., 2019). Moreover, phylogenetic analysis has demonstrated that HipT and HipA toxins are relatively closely related within a very broad superfamily of HipA-homologous kinases (Gerdes et al., 2021). To gain further insight into the basis of this important difference, we initially performed a focused phylogenetic analysis of the 48 HipT orthologues identified so far among bacterial genomes (Gerdes et al., 2021). Alignment and clustering reveals a largely bifurcated, phylogenetic tree for which one large branch (the "SIS group") contains HipT kinases with two serine residues in close proximity in the Gly-rich loop corresponding to the known autophosphorylation sites in HipT of E. coli O127:H6 (Figure 1a). In the remainder of the HipT orthologues, this region contains variable Ser/Thr motifs, including TxT, Sxx, xxT, SxT, and 


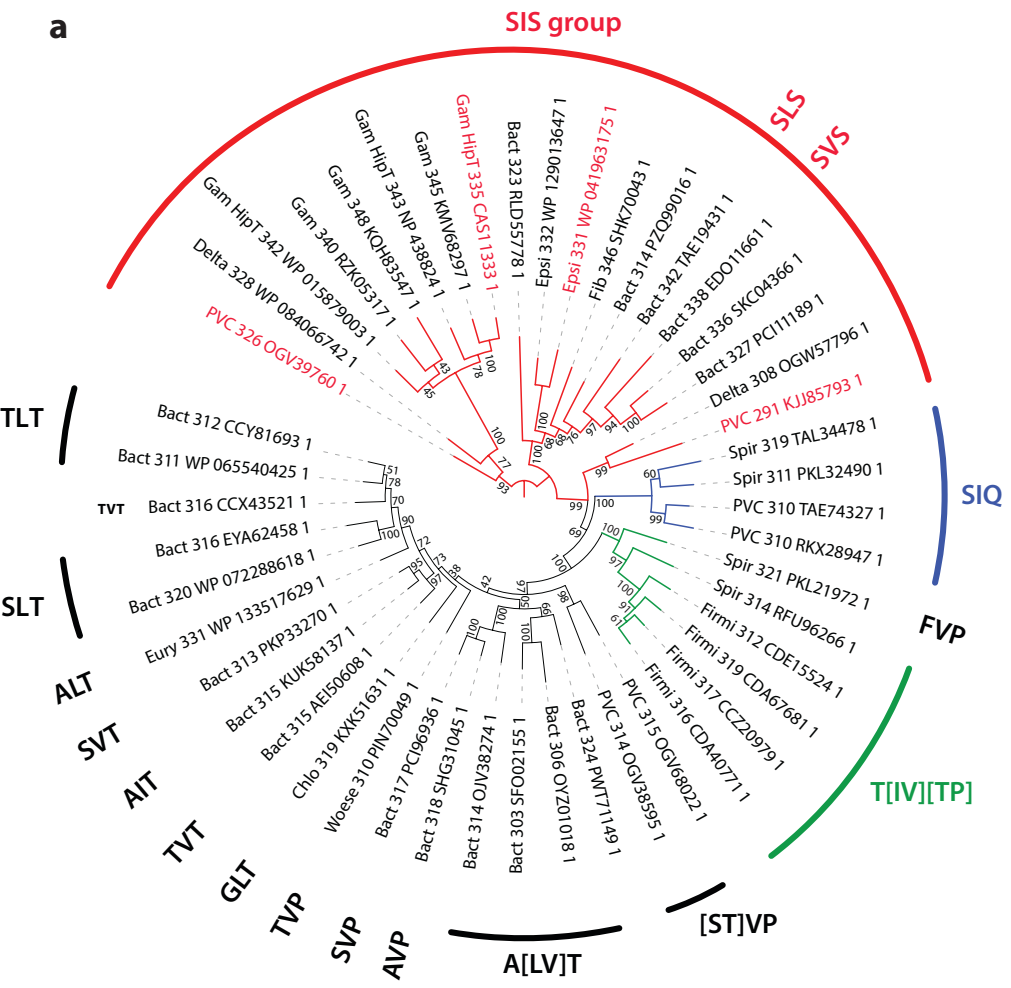

b

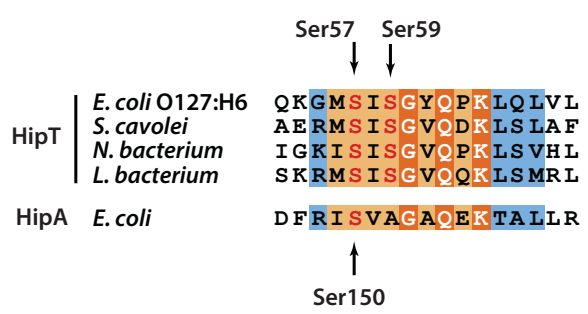

c

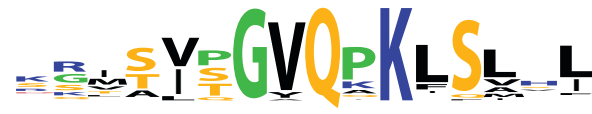

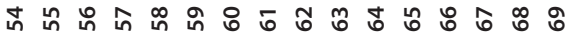

d

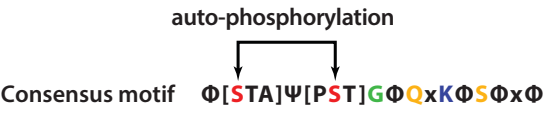

Figure 1. Phylogenetic analysis of HipT. a. Phylogenetic guide tree of $48 \mathrm{HipT}$ orthologues with sequences motifs (potential phosphorylation sites) indicated on the side. The "SIS group" (red) is by far the largest group followed by the T[IV][TP] group (green), and SIQ group (blue). Sequences used in the alignment in $\mathbf{b}$ are shown with red letters. Numbers on the branches indicate bootstrap confidence levels. b. Sequences of the Gly-rich loop (orange background), including the potential phosphorylation sites for selected HipT orthologues compared to HipA from E. coli K-12. Known phosphorylation sites in E. coli O127:H6 HipT (top) and E. coli K-12 HipA (bottom) are indicated with arrows and conserved sequence motifs with bold white text. c. Sequence logo for the Gly-rich loop derived from all 48 HipT sequences. d. Consensus motif for the HipT Gly-rich loop with known phosphorylation sites in red. $\Phi$ indicates a hydrophobic residue, while $\Psi$ are aliphatic residues. 
Txx. We note that the single position between the two Ser/Thr residues invariably is occupied by a small hydrophobic residue (I, L, or V) and that a small subbranch close to the SIS group represents an interesting variation with a Gln in the second autophosphorylation position (the "SIQ" group). The remainder of the Gly-rich loop across a range of HipT kinases is highly similar to Hip $\mathrm{Ac}_{\mathrm{Ec}}$, which has the sequence I $\underline{S}$ A $\underline{A G A Q E K}$ (Figure 1b-c). We can thus derive a consensus motif for the HipT Glyrich loop, which includes several conserved hydrophobic positions in addition to the two Ser/Thr residues (Figure 1d). In summary, phylogenetic analysis reveals that a large fraction of HipT kinases contain a conserved SIS motif with two potential phosphorylation positions in their Gly-rich loops but also that this region displays some sequence diversity, which may point to subtle differences in regulation.

\section{HipBST forms a hexameric higher-order complex}

111 Comparison of the HipBST and HipBA sequences reveals that HipS shows similarity to the N-

112 terminal subdomain 1 of HipA (Figure S1 and S2a) and that both HipB and HipT of hipBST contain

113 N-terminal extensions of around 35 residues not found in HipB and HipA of hipBA, respectively

114 (Figure 2a and S1). To understand the molecular basis for the functional and structural differences,

115 we determined the crystal structure of HipBST from enteropathogenic E. coli O127:H6 expressed 116 with inactive HipT (D233Q) to $2.9 \AA$ by molecular replacement using Hip $\mathrm{A}_{\mathrm{Ec}}$ as search model 117 (Figure 2b). The refined structure $\left(\mathrm{R}=19.5 \% / \mathrm{R}_{\text {free }}=22.8 \%\right)$ has two copies of each of the three 118 proteins in the asymmetric unit and is generally well-defined for HipB and HipT, while HipS appears 119 to be more flexible. Analysis by the protein interaction server, PISA (Krissinel \& Henrick, 2007), suggests that the biological assembly is comprised of a dimer of HipBST heterotrimers, which are

121 held together through dimerisation of HipB (Figure 2b). This is consistent with the observation that 
a

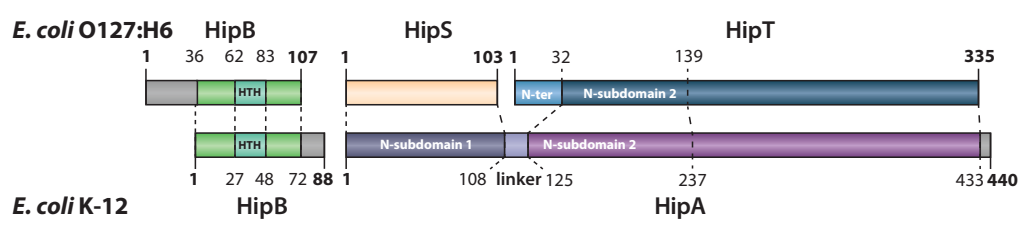

b

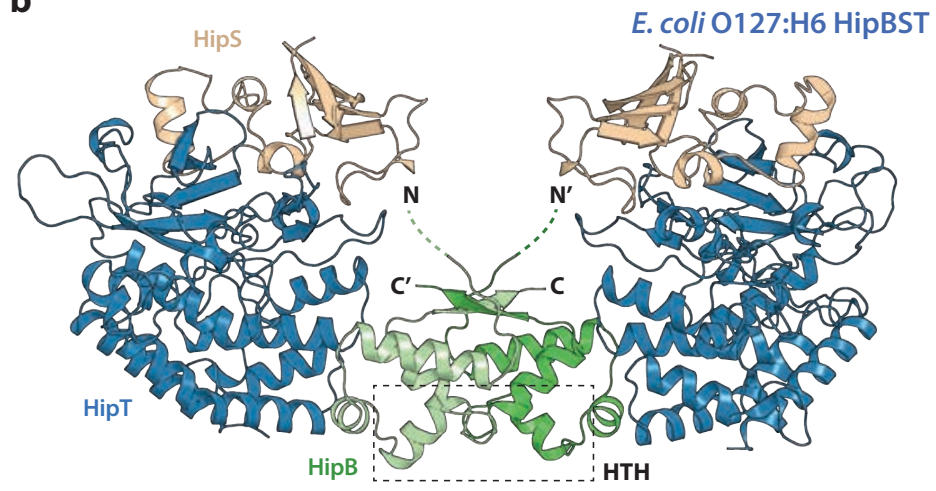

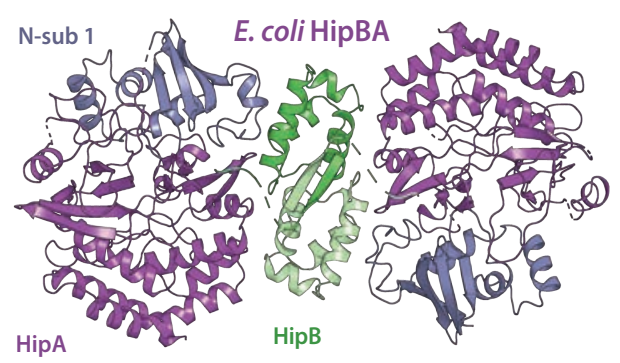

e

d
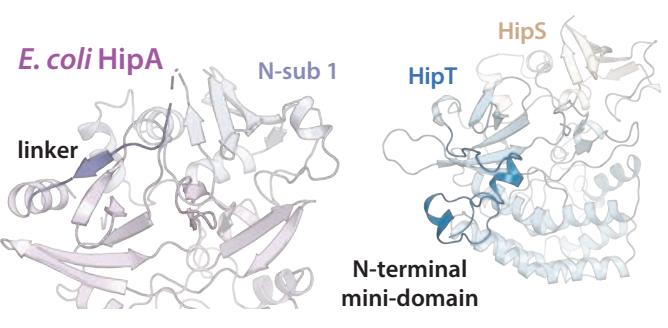

Figure 2. Crystal structure of the $E$. coli O127:H6 HipBST complex. a. Schematic representation of $E$. coli O127:H6 HipBST and canonical E. coli HipBA showing corresponding proteins and domains. Dashed lines indicate similar domains and grey areas represent regions missing domains in the crystal structures. HipS (beige) is structurally similar to the $\mathrm{N}$-subdomain 1 of HipA (dark purple), HipT has an additional N-terminal mini-domain not found in HipA (light blue) while HipA has a short linker between the two N-subdomains (light purple). b. $E$. coli O127:H6 HipBST forms a hetero-hexameric complex with HipS (beige) on top of HipT (blue) through dimerisation of HipB (green) also generating a helix-turn-helix DNA-binding motif (HTH, dashed box). c. Crystal

132 structure of the E. coli K-12 HipBA complex (PDB: 2WIU) shown as cartoon with HipA in blue purple (N-terminal 133 subdomain 1) and purple and the HipB homodimer in two shades of green (Evdokimov et al., 2009). d. The linker 134 between $\mathrm{N}$-subdomain 1 and 2 (dark purple) physically linking the two domains in Hip $\mathrm{A}_{\mathrm{Ec}}$. e. The N-terminal mini135 domain of HipT (blue), which is absent from HipA. 
138 In this complex, the two HipB monomers interact strongly (interface area $=1,405 \AA^{2}$ ) while the 139 interface between HipB and HipT is relatively small $\left(513 \AA^{2}\right)$. The biological relevance of the hetero140 hexamer is supported by analytical gel filtration (Figure S2b) and small-angle X-ray scattering 141 (SAXS, Figure S6), the latter of which showed a major species with a total mass of $129 \pm 8 \mathrm{kDa}$, in 142 good agreement with the theoretical mass of $125.6 \mathrm{kDa}$. HipB consists of an $\alpha$-helical bundle of four 143 helices including the predicted DNA-binding HTH motif (residues 62-83), which forms a canonical 144 HTH DNA-binding domain exposed at the bottom of the hetero-hexamer (Figure 2b). The helical 145 bundle is followed by a small $\beta$ strand that forms an antiparallel sheet through pairing with the 146 corresponding region in the neighbouring molecule of HipB. The N-terminal extension of HipB (36 147 residues) is not visible in the electron density, but secondary structure prediction indicates that it 148 forms a long $\alpha$-helix (Figure S1a). HipS is closely associated with HipT and consists of a solvent149 facing, five-stranded, anti-parallel $\beta$ sheet of which four strands are located in the $\mathrm{N}$ terminus 150 (residues 1-52). These are followed by a small domain of three helices (residues 53-94) that forms 151 the interface to HipT followed by the fifth $\beta$ strand (residues 95-102, Figure 2b and S3a).

153 The overall architecture of the HipBST complex consists of two HipST toxin:antitoxin complexes 154 separated in space by the dimer of the HipB and in this way differs markedly different from HipBA $\mathrm{Ec}_{\mathrm{Ec}}$ 155 in which two HipA toxins are closely packed head-to-tail around two HipB antitoxins (Figure 2c). 156 This configuration is key to the proposed way in which HipB can function as antitoxin, namely by 157 restricting the movement of the N-terminal subdomain of HipA (Figure 2c, blue) (Schumacher et al., 158 2009). As suggested from sequence analysis, HipS bears a striking structural resemblance to the N159 terminal subdomain 1 of HipA (Schumacher et al., 2012), both with respect to its overall fold and 160 placement in relation to HipT. As a result of this, the HipS C-terminus overlaps with a extended linker 161 that bridges N-subdomain 1 to $\mathrm{N}$-subdomain 2 in HipA (Figure 2d). HipT corresponds structurally 
to the core kinase fold of HipA, except for the presence of an additional N-terminal mini-domain (residues 1-41), the role of which is possibly to anchor N-subdomain 2 (residues 42-140) to the core of the kinase in the absence of the physical coupling to HipS (Figure 2a and 2e). Interestingly, the overall structure of the HipBST complex is reminiscent of the structure of a HipBA complex from Shewanella oneidensis, which was crystallised in complex with DNA (Figure S2c) (Wen et al., 2014). This suggests that the open conformation is not a specific result the tripartite configuration and superpositioning of the DNA-binding domains (Figure S3b) and analysis of potential interactions with DNA (Figure S3c) suggests HipBST could bind DNA in a similar way.

\section{HipT has an inactive conformation inside the HipBST complex}

For Hip $\mathrm{A}_{\mathrm{Ec}}$, two discrete active site conformations are observed depending on phosphorylation state and whether substrate (ATP) is bound or not (Schumacher et al., 2015; Schumacher et al., 2012; Schumacher et al., 2009). In its non-phosphorylated form and in the presence of ATP, the flexible Gly-rich loop is found in an inward conformation in which main chain amino groups on Ala154 and Gln155 coordinate the ATP phosphate groups (Figure 3a) (Schumacher et al., 2009). Phosphorylation of Ser150, however, causes ejection of the loop through strong interactions of the phosphate group with active sites residues, which prevents binding of ATP (Figure 3b) (Schumacher et al., 2012). In the HipBST crystal structures, we find the Gly-rich loop of HipT forms a short $\alpha$ helix and is in a conformation most similar to the ejected form of the Hip $\mathrm{A}_{\mathrm{Ec}}$ loop despite HipT not being phosphorylated (Figure 3c). In this conformation, Ser59, which is known to undergo autophosphorylation in HipT, forms interactions to several active site residues, including Asp210 and the Ser57 auto-phosphorylation site (Vang Nielsen et al., 2019). We conclude that inside the HipBST complex, HipT in its non-phosphorylated state is maintained in an inactive conformation that does not support ATP binding. 
a

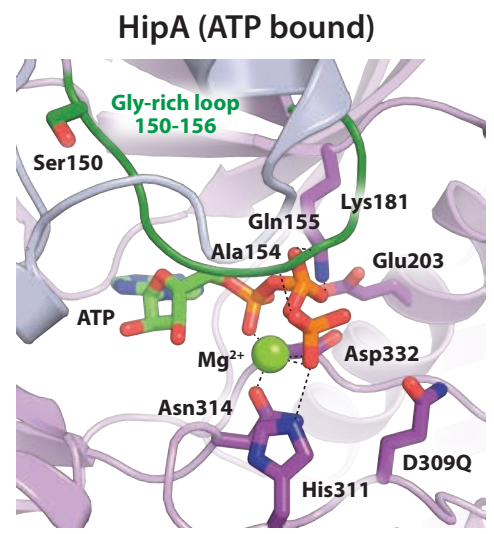

b

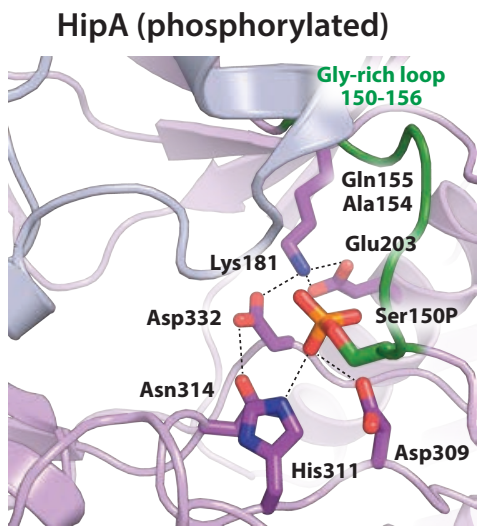

C

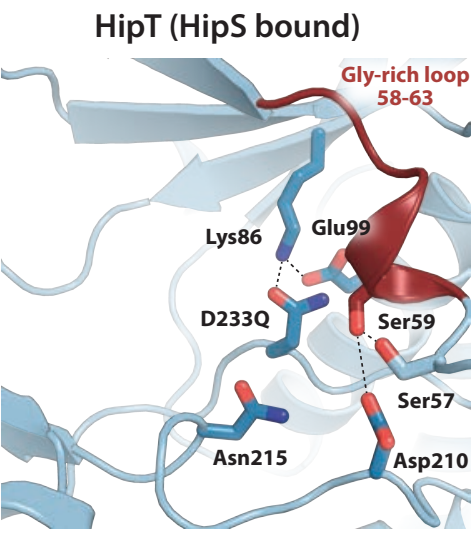

d
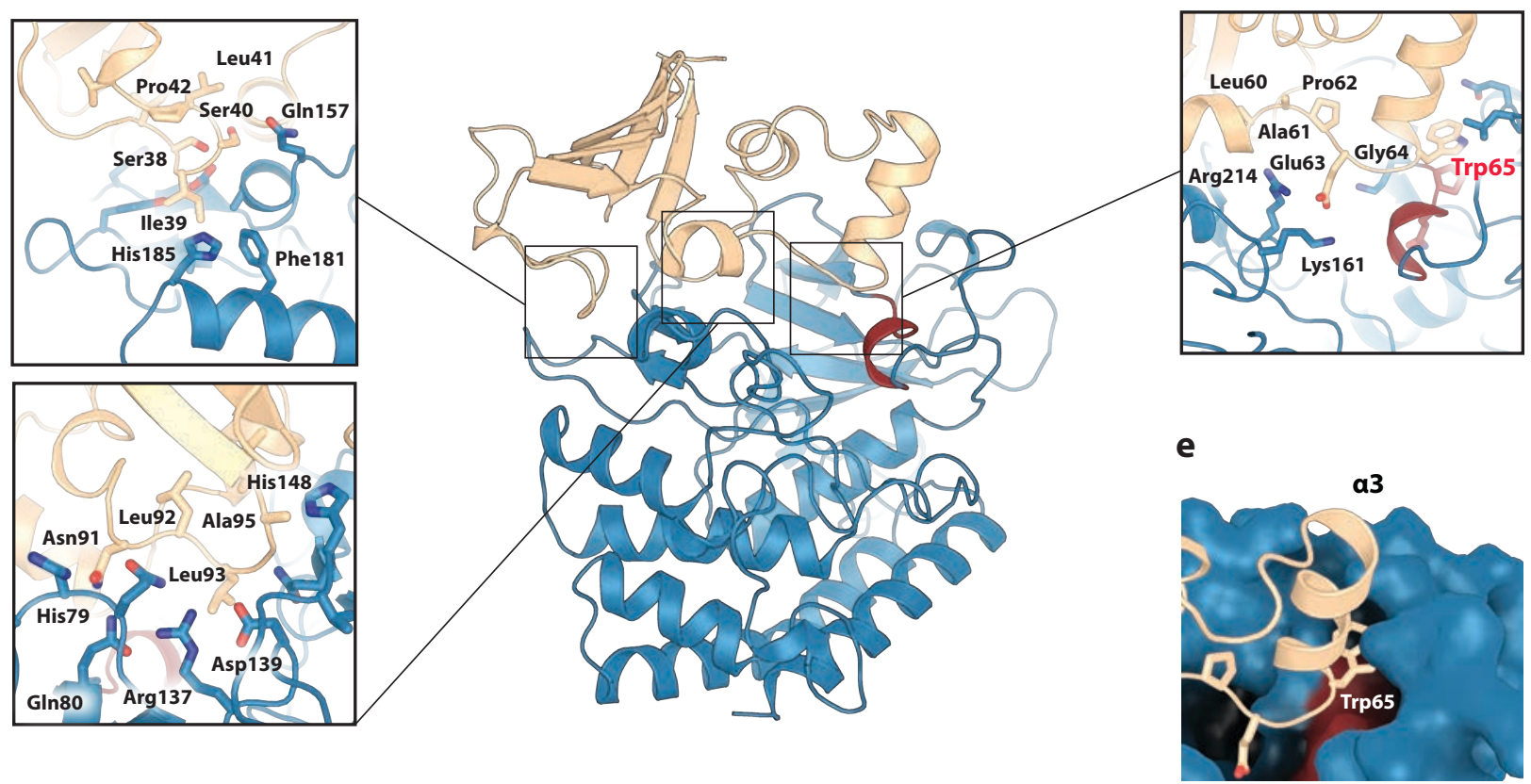

f
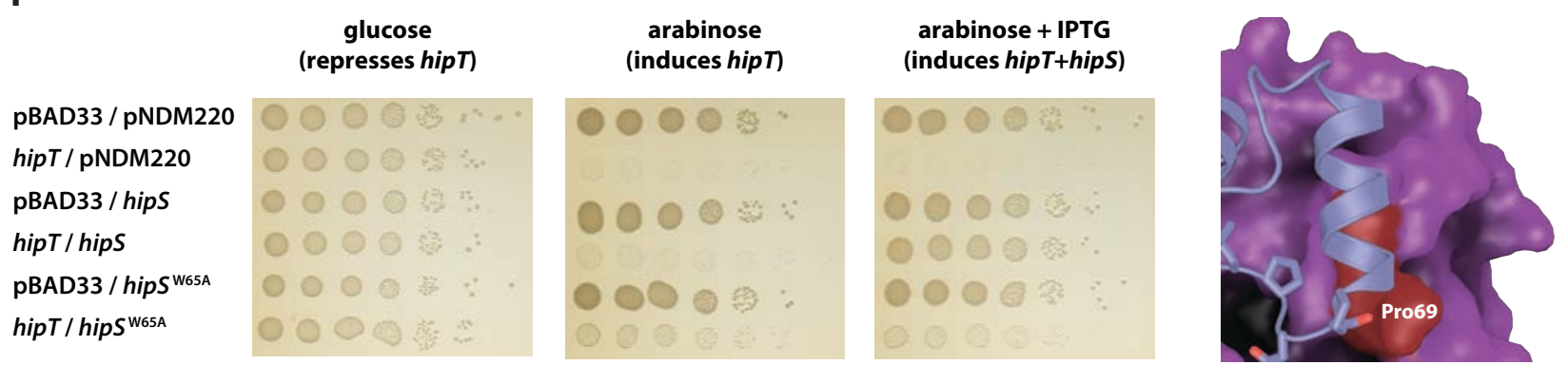

Figure 3. Trp65 is essential for HipS to function as antitoxin. a. Overview of the HipA active site, bound to ATP

189 (green, PDB: 3DNT) (Schumacher et al., 2009). Residues interacting with the nucleotide are highlighted as well as the Gly-rich loop (residues 150-156, green) including Ser150. D309Q is a mutated active site residue. b. The active site of HipA phosphorylated on Ser 150 (green/orange, PDB: 3TPE) with the Gly-rich loop (green) in the outward- 
192 facing conformation (Schumacher et al., 2012). c. The active site of HipBS-bound HipT ${ }^{\mathrm{D} 233 \mathrm{Q}}$ with the Gly-rich loop

193 (residues 58-63, ruby) in the outward-facing conformation. D233Q is the mutated active site residue. d. Overview 194 and detailed interactions between HipS (beige) and HipT (blue) with its Gly-rich loop (ruby) and interacting residues 195 indicated. e. Top, HipS Trp65 (beige) is placed in a pocket on the surface of HipT (blue); Bottom, the corresponding 196 residue (Pro69) in HipA (purple, PDB: 3TPD). f. E. coli MG1655 harbouring empty pBAD33 vector ("pBAD33") 197 or pSVN1 (pBAD33::hipT) in combination with empty pNDM220 vector ("pNDM220"), pSVN109 198 (pNDM220::hipS), or pSVN178 (pNDM220::hipS ${ }^{\mathrm{W} 65 \mathrm{~A}}$ ) as indicated. Plates contained $0.2 \%$ glucose (to repress 199 hipT), $0.2 \%$ arabinose (to induce hipT), or $0.2 \%$ arabinose plus $200 \mu \mathrm{M}$ IPTG (to induce hipS, or hipS ${ }^{\mathrm{W} 65 \mathrm{~A}}$ ). The 200 plates are representative of at least three independent experiments. 
HipS inhibits HipT through insertion of a hydrophobic residue near the active site

To understand how HipS, which is homologous to the N-terminal subdomain 1 of HipA, can function

as the antitoxin in the HipBST system, we then focused on the specific interactions found between

HipS and HipT. There are three major areas of contact between the two proteins with several strong interactions on both sides of the kinase domain, involving both hydrogen bonds and hydrophobic and charged interactions (Figure 3d). Near the Gly-rich loop, HipS Glu63 points directly into the HipT active site while Trp65 is wedged into a deep cavity formed by the HipT Gly-rich loop in its outward conformation. While Glu63 is conserved in the N-terminal subdomain of HipA $A_{E c}$ (Asp67) and therefore less likely contribute to functional differences between the two systems, the Trp65 motif

212 (Figure 3e, top) is structurally different from the corresponding interaction in Hip $\mathrm{A}_{\mathrm{Ec}}$, which has the helix (HipS $\alpha 3$, Figure S2a) in a different orientation and a proline residue at this position (Figure 3e, bottom). Moreover, Trp65 is conserved in many HipS orthologues (Figure S2a). We hypothesised that placement of the bulky Trp residue inside HipT might block transition of the Glyrich loop from the outward to the inward conformation, likely required for ATP binding. To test this,

217 we mutated the Trp65 in HipS to alanine and tested the ability of the antitoxin to antagonise the kinase in E. coli $\mathrm{K}-12$ on both solid media and in liquid culture (Figure $3 \mathbf{f}$ and S3d). In both experiments, absence of the bulky Trp65 side chain strongly reduced the antitoxin activity of HipS, confirming that this interaction is critical to inhibition of HipT. We conclude that Trp65 is essential for the ability of HipS to inhibit HipT, presumably either by increasing the affinity of HipS for HipT, or by preventing the Gly-rich loop from adopting the conformation required for ATP binding.

\section{The conformation of HipT in the HipBST complex is independent of phosphorylation}


individually but not concomitantly (Vang Nielsen et al., 2019). To understand the individual roles of the two autophosphorylation positions, we next determined crystal structures of two HipBST cases, the remaining serine residue (i.e. Ser57 in HipBST ${ }^{\mathrm{S} 59 \mathrm{~A}}$ and Ser59 in HipBST ${ }^{\mathrm{S} 57 \mathrm{~A}}$ ) had become phosphorylated prior to crystallisation (Figure 4a and S4a-b). For HipBST ${ }^{\mathrm{S} 59 \mathrm{~A}}$, phosphorylation of Ser57 was incomplete and could only be modelled in one of the two HipT molecules in the asymmetric unit (Figure S4b). The phosphate group of Ser59 (P-Ser59) forms strong interactions to Lys161, His212, and the catalytic Asp210 inside the active site (Figure 4a, top), while P-Ser57 is located further away from the active site and forms hydrogen bonds to Tyr162 and Asp210 (Figure 4a, bottom). In both structures, however, the Gly-rich loop maintains its outward conformation, incompatible with ATP binding, suggesting that HipT is inactive. The phosphate group on Ser59 occupies a position very similar to phosphorylated Ser150 in $\mathrm{Hip} \mathrm{A}_{\mathrm{Ec}}$, despite the fact that this residue aligns to Ser57 at the sequence level (Figure S4c, top and 1b). This position structurally overlaps with the $\gamma$ phosphate of ATP in the structure of ATP-bound Hip $\mathrm{Ac}_{\mathrm{Ec}}$, suggesting that phosphorylation prevents nucleotide binding in the kinase active site (Figure 4b). Intriguingly, the phosphoryl group of P-Ser57 overlaps structurally with that of phosphorylated Ser147 in HipAso, which corresponds to $\mathrm{HipA}_{\mathrm{Ec}}$ Ser150 at the sequence level (Figure S4c, bottom). In order to confirm the phosphorylation state of the HipT variants in vitro, we analysed purified HipBST complexes on Phos-tag SDS-PAGE gels, which contain functional molecules that cause retention of phosphorylated protein species in the gel (Figure 4c, top). Here, we observed a marked difference in migration between HipT from wildtype HipBST, HipBST ${ }^{\mathrm{S} 59 \mathrm{~A}}$, and $\mathrm{HipBST}^{\mathrm{S} 57 \mathrm{~A}}$ confirming that $\mathrm{HipT}^{\mathrm{S} 57 \mathrm{~A}}$ is predominantly phosphorylated while $\operatorname{Hip}^{\mathrm{S} 59 \mathrm{~A}}$ is only partially modified, consistent with the crystal structures. 
a

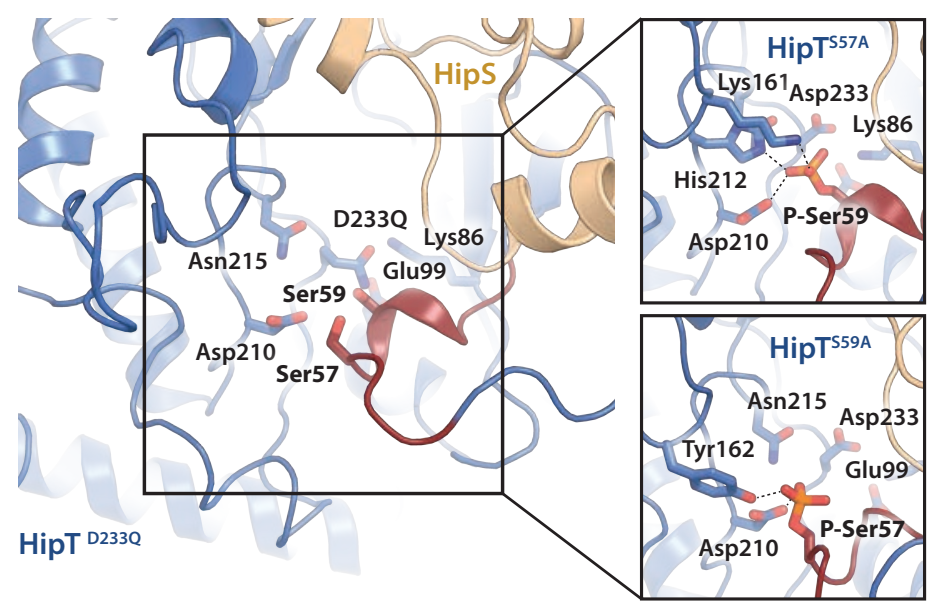

b

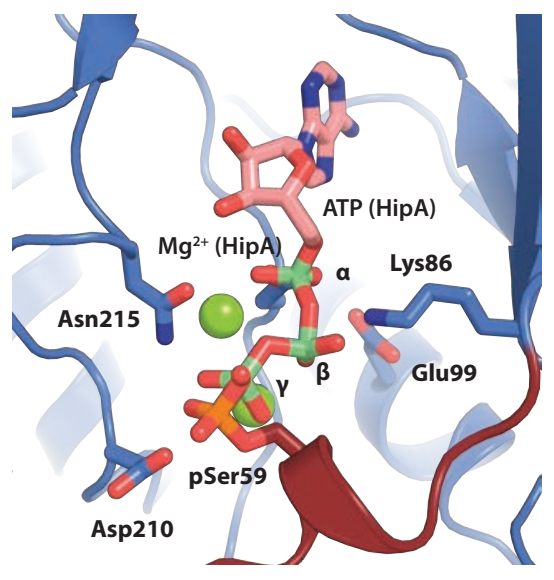

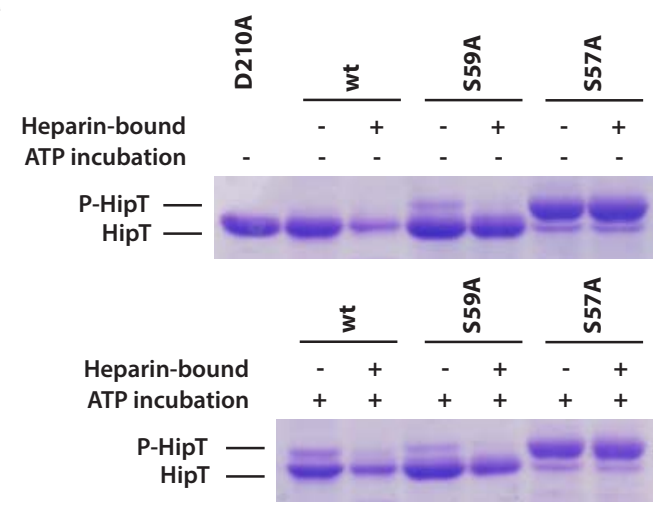

e

hipt (SIS, wt) hipT(DIS) hipt(SID) hipt(DID) hipT(DIA)

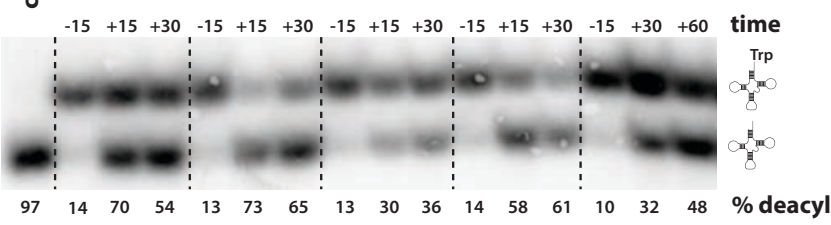

d
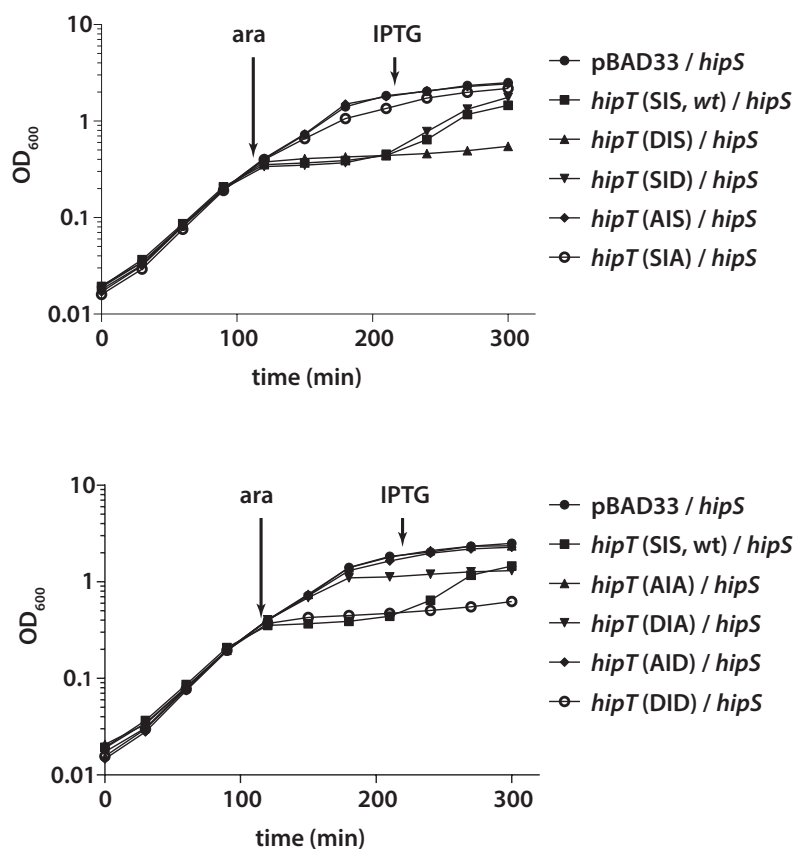

Figure 4. The phosphoserine positions in HipT have distinct functional roles. a. Overview of the HipT kinase active site in the D233Q mutant as well as S57A (top) and S59A (bottom) structures. The phosphate groups on Ser57 (in S59A) and Ser59 (in S57A) are shown in orange and relevant nearby residues are highlighted. b. Closeup of the HipT active site as observed for the S57A mutant with relevant active site residues shown as sticks. The Gly-rich loop is shown in red with pSer59 indicated. Overlaid is ATP and $2 \mathrm{Mg}^{2+}$ ions derived from the structure of HipA (PDB: 3DNT, salmon/green) (Schumacher et al., 2009). c. HipT bands on Phos-tag gels (stained by Coomassie Blue) of purified HipBST complex before (-) and after $(+)$ a Heparin column step to separate various complex species, as well as before (-) and after (+) treatment with $10 \mathrm{mM}$ ATP, as indicated. Phosphorylated ("P- 
261 HipT") and non-phosphorylated ("HipT") protein species are indicated. The gels are representative of two 262 independent experiments. d. Top, growth curves measured by $\mathrm{OD}_{600}$ of E. coli MG1655 harbouring single 263 autophosphorylation mutants pSVN194 (pBAD33::hipT $T^{\mathrm{S} 57 \mathrm{D}}$, "hipT (DIS)"), pSVN195 (pBAD33::hipT ${ }^{\mathrm{S} 59 \mathrm{D}}$, "hipT

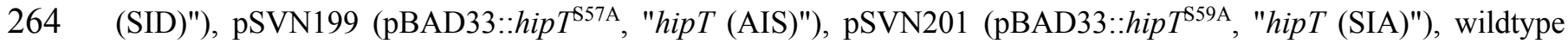
265 HipT pSVN1 (pBAD33::hipT, "hipT (SIS, wt)"), or the empty pBAD33 vector, as indicated, in combination with pSVN109 (pNDM220::hipS). hipT (ara) and hipS (IPTG) expression were induced at the indicated time points. The curves show the mean values of results from at least two independent experiments with error bars indicating standard deviations (hidden when small); Bottom, growth curves of E. coli MG1655 harbouring double autophosphorylation

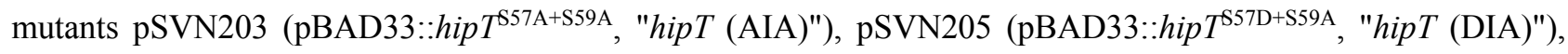

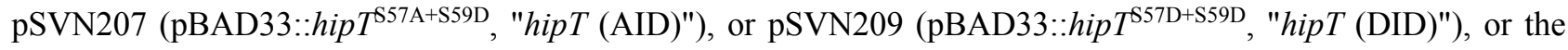
empty pBAD33 vector, as indicated, in combination with pSVN109 (pNDM220::hipS). hipT (ara) and hipS (IPTG) expression were induced at the indicated time points. The curves show the mean values of results from at least two independent experiments with error bars indicating standard deviations (hidden when small). e. Northern blot analysis probing against $\operatorname{tRNA}^{\text {trpT }}$ based on RNA extracted from E. coli MG1655 harbouring pSVN110 (pNDM220::hipB-S) in combination with pSVN1 (pBAD33::hipT, "hipT ( $\left.\left.\mathrm{S}^{57} \mathrm{IS}^{59}, w t\right) "\right)$, pSVN194

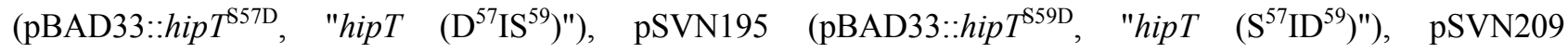

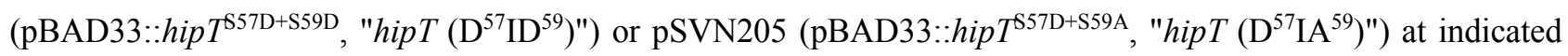
time points in minutes before (-) and after $(+)$ addition of arabinose $(0.2 \%)$ to induce wildtype hipT or autophosphorylation mutants. Quantification of the bands (represented as \% deacylated tRNA) is shown below the gel. The control ("ctrl") is chemically deacylated tRNA. The percentage of deacylated tRNA (bottom) was based on quantification of total tRNA ${ }^{\text {trpT }}$. Results from strains harbouring pSVN1 (pBAD33::hipT), pSVN194

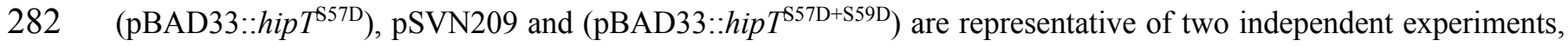

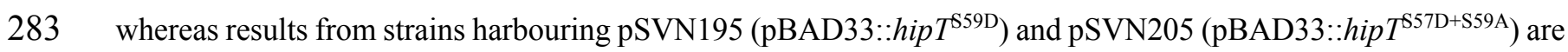
284 from single experiments. 
but incubation with ATP for 30 minutes leads to a visible increase in phosphorylation of wildtype HipT while no further phosphorylation seemed to take place for the mutants upon incubation with

ATP (Figure 4c, bottom). In summary, we conclude that the conformation of the HipT active site loop (Gly-rich loop) inside the HipBST complex is independent of the autophosphorylation state.

The two phosphoserine positions in HipT have separate functional roles

We next sought to understand the functional implications of autophosphorylation of HipT at the two positions. To this end, we designed a set of HipT mutants with either Ala (to prevent autophosphorylation) or Asp (to mimic the phosphorylated state) in one or both of the two Ser positions. Liquid culture assays showed that both wildtype HipT $\mathrm{S}^{57} \mathrm{IS}^{59}$ and HipT $\mathrm{S}^{57} \mathrm{ID}^{59}$ caused growth inhibition upon overexpression in E. coli $\mathrm{K}-12$ and could be repressed by concomitant expression of HipS (Figure 4d, top) (Vang Nielsen et al., 2019). In contrast to this, growth inhibition by the HipT $\mathrm{D}^{57} \mathrm{IS}^{59}$ variant could not be repressed upon induction of HipS. Moreover, mutation to alanine at either position caused very little $\left(\mathrm{S}^{57} \mathrm{IA}^{59}\right)$ or no $\left(\mathrm{A}^{57} \mathrm{IS}^{59}\right)$ growth inhibition, suggesting that the serine residues or their phosphorylated counterparts are important for HipT activity on the target TrpS (Figure 4d, top). To rule out any effects of autophosphorylation of the non-mutated Ser position, we created double mutants for which both serine residues were mutated into either alanine or aspartate (Figure 4d, bottom). Of these, HipT $\mathrm{D}^{57} \mathrm{ID}^{59}$ causes immediate growth inhibition while HipT $\mathrm{D}^{57} \mathrm{IA}^{59}$ shows a similar but delayed phenotype, and consistent with the observation above, neither could be repressed by HipS induction. And as before, placing alanine at either position either 307 completely abolished $\left(\mathrm{A}^{57} \mathrm{IA}^{59}\right.$ and $\left.\mathrm{A}^{57} \mathrm{ID}^{59}\right)$ or strongly delayed $\left(\mathrm{D}^{57} \mathrm{IA}^{59}\right)$ growth inhibition. To rule out any effects due to the fact that HipB is missing in this setup, we finally repeated the experiments 
312 To confirm that the growth inhibition observed was in fact due to phosphorylation and inactivation

313 of TrpS, we took samples from E. coli cultures both before and at several time points after induction 314 of wildtype HipT as well as all variants that caused markedly growth inhibition, extracted total RNA, 315 and performed Northern blotting using probes against tRNA ${ }^{\text {trpT }}$ (Figure 4e) as well as tRNA gltTUVW $^{\text {sig }}$ 316 and tRNA ${ }^{\operatorname{argVYZQ}}$ (Figure S4f) as controls. Here, charging of the tRNA is visible as a shift of the 317 probed RNA band and thus allows direct assessment of the activity of TrpS in vivo in the presence of 318 the various HipT variants. Induction of all variants caused accumulation of deacylated tRNA ${ }^{\text {trpT }}$ 319 (Figure 4e) but no accumulation of the two control tRNAs (Figure S4f), which strongly suggests 320 that the observed growth effects are due to reduction in the cellular pool of acylated tRNA. Taken 321 together, we conclude that HipT autophosphorylation at Ser57 prevents HipS inhibition and does not 322 prevent phosphorylation of the target, TrpS. On the other hand, phosphorylation at Ser59 does not 323 affect HipS inhibition, indicating that phosphorylation at the two positions serve separate, functional 324 roles.

$h i p B$ is both required and sufficient for transcriptional repression

327 To understand the functional role of HipB, specifically how DNA binding has been separated from 328 the antitoxin function in the tripartite hipBST system, we initially constructed a vector-based 329 transcriptional fusion reporter, in which the native hipBST promoter region and 5 ' region of the hipB 330 gene was transcriptionally fused to lacZ (Figure 5a, right). We then expressed various combinations 331 of hipB, hipS, and hipT in trans in E. coli MG1655 $\triangle$ lacIZYA cells that do not harbour the hipBST 332 operon, utilising two inactivating kinase mutations, $h i p T^{\mathrm{D} 210 \mathrm{Q}}$ (Figure 5a, left) and hipT $^{\mathrm{D} 233 \mathrm{Q}}$ (Figure 333 S5a) to avoid toxicity. In both cases, in the absence of expressed hipBST components, active 334 transcription could be observed by the blue colour of the colonies (Figure 5a, left, top row, pBAD33), 
bioRxiv preprint doi: https://doi.org/10.1101/2022.01.28.478185; this version posted January 31,2022 . The copyright holder for this preprint (which was not certified by peer review) is the author/funder, who has granted bioRxiv a license to display the preprint in perpetuity. It is made available under aCC-BY 4.0 International license.

a
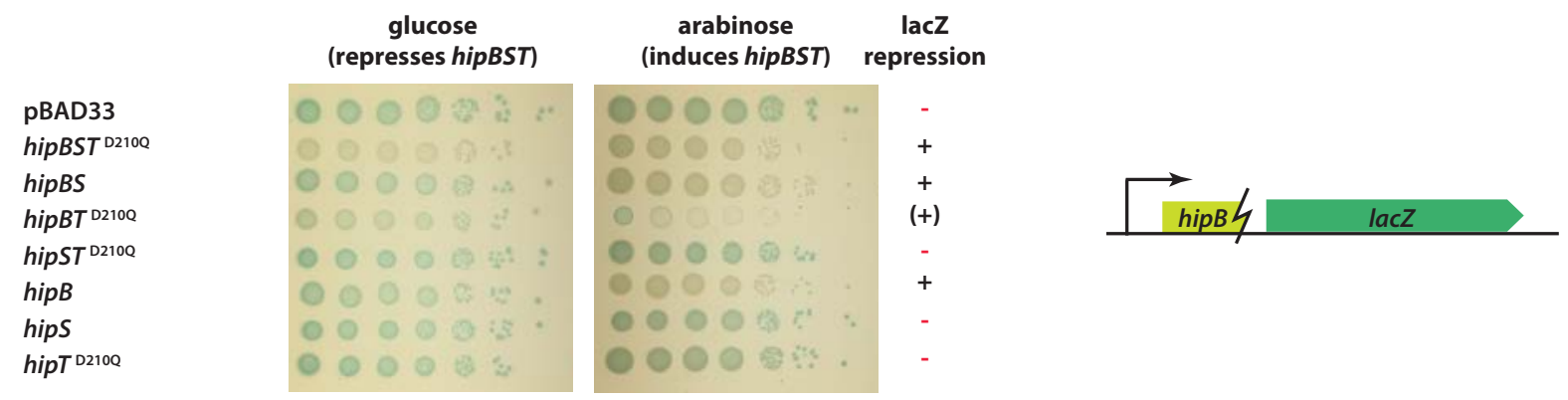

b

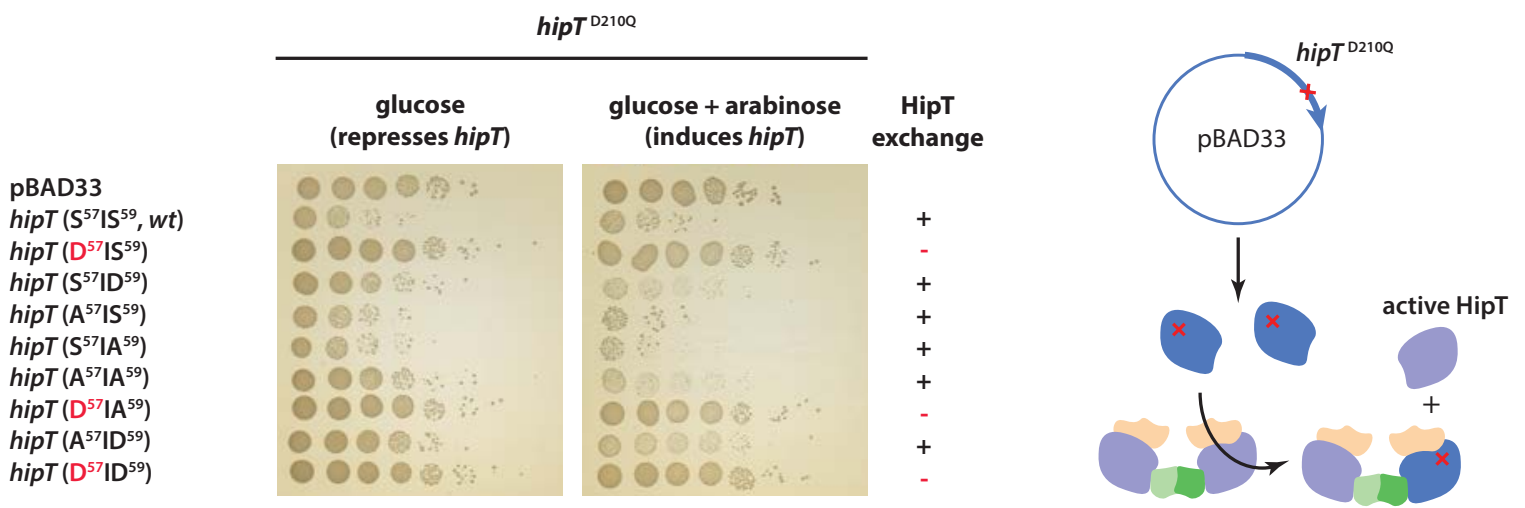

C

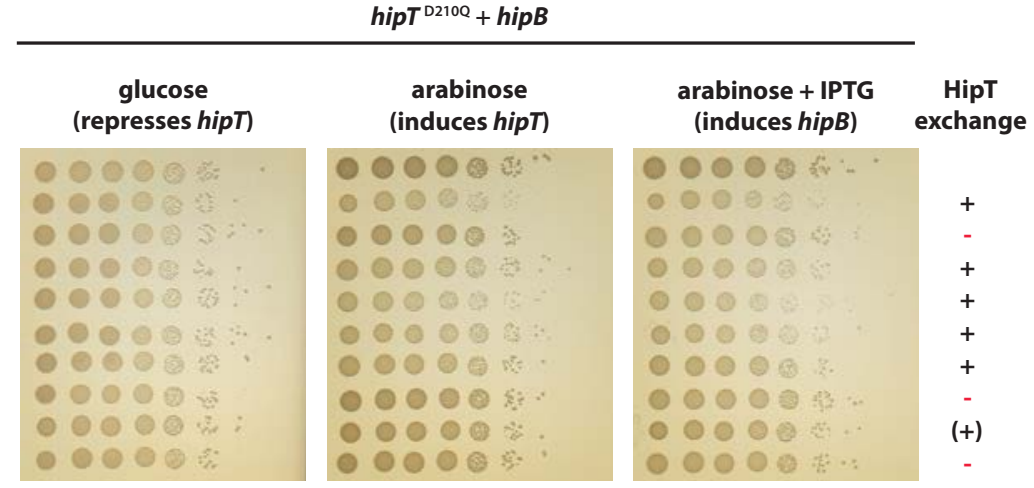

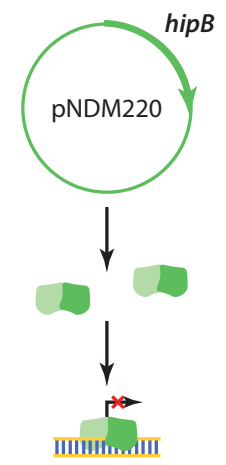

337 Figure 5. The HipBST complex is dynamic in vivo. a. Right, transcriptional reporter assay using a plasmid with

338 the hipBST promoter region and a 5 ' fragment of the hipB gene (including 224 bp upstream of hipB plus the first 73

339 bp of the hipB gene, shown with a lightening symbol) transcriptionally fused to lacZ. Left, E. coli TB28 harbouring

340 the reporter plasmid (pSVN141, pGH254:: $\mathrm{P}_{\text {hipBST }}$-hipB'-lacZ) and empty pBAD33 vector or combinations of hipB,

341 hipS, and hipT in the context of the HipT ${ }^{\mathrm{D} 210 \mathrm{Q}}$ inactive mutant; pSVN180 (pBAD33::hipB-S-T ${ }^{\mathrm{D} 210 \mathrm{Q}}$, "hipBST ${ }^{\mathrm{D} 210 \mathrm{Q}}$ ),

342 pSVN182 (pBAD33::hipB-S, "hipBS"), pSVN184 (pBAD33::hipB-T ${ }^{\mathrm{D} 210 \mathrm{Q},} \quad$ "hipBT 2 210Q"), pSVN187

343 (pBAD33::hipS-T ${ }^{\mathrm{D} 210 \mathrm{Q}}$, "hipST ${ }^{\mathrm{D} 210 \mathrm{Q} ")}$, pSVN189 (pBAD33::hipB, "hipB"), pSVN190 (pBAD33::hipS, "hipS"), or

344 pSVN192 (pBAD33::hipT ${ }^{\mathrm{D} 210 \mathrm{Q}}$, "hipT $\left.{ }^{\mathrm{D} 210 \mathrm{Q}}\right)$ were grown, diluted, and spotted onto YT, X-gal agar plates 
containing $0.2 \%$ glucose (to repress $h i p B / S / T^{\mathrm{D} 210 \mathrm{Q}}$ ), or $0.2 \%$ arabinose (to induce $h i p B / S / T^{\mathrm{D} 210 \mathrm{Q}}$ ). Results are

346 representative of two independent experiments. b. Left, HipT exchange assay where overexpression of a catalytically inactive kinase (HipT $\left.{ }^{\mathrm{D} 210 \mathrm{Q}}\right)$ is used to exchange wild type HipT from the HipBST complex in vivo.

348 Right, spot assays of E. coli $\mathrm{O} 127$ harbouring the empty pBAD33 vector, pSVN220 (pBAD33::hipT ${ }^{\mathrm{D} 210 \mathrm{Q}}$, "hipT

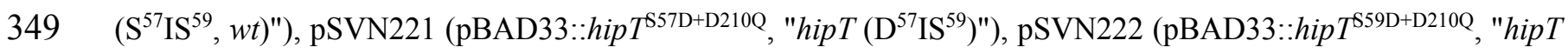
$\left.350\left(\mathrm{~S}^{57} \mathrm{ID}^{59}\right) "\right)$, pSVN223 (pBAD33::hipT ${ }^{\mathrm{S} 57 \mathrm{~A}+\mathrm{D} 210 \mathrm{Q}}$, "hipT $\left.\left(\mathrm{A}^{57} \mathrm{IS}^{59}\right) "\right)$, pSVN224 (pBAD33::hipT ${ }^{\mathrm{S} 59 \mathrm{~A}+\mathrm{D} 210 \mathrm{Q}}$, "hipT

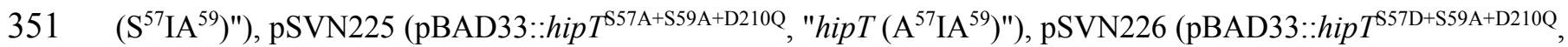

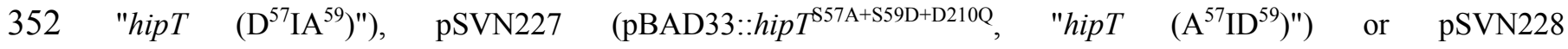
353 (pBAD33::hip $T^{\mathrm{S} 57 \mathrm{D}+\mathrm{S} 59 \mathrm{D}+\mathrm{D} 210 \mathrm{Q}}$, "hipT $\left.\left(\mathrm{D}^{57} \mathrm{ID}^{59}\right) "\right)$ on YT agar plates containing $0.2 \%$ glucose (to repress hipT ${ }^{\mathrm{D} 210 \mathrm{Q}}$ 354 autophosphorylation mutants) or $0.2 \%$ glucose plus $0.2 \%$ arabinose to induce a limited amount of HipT. The column 355 to the right indicates whether HipT exchange has taken place $(+)$ or not (-). Results are representative of two 356 independent experiments. c. As b but with additional overexpression of hipB to avoid renewed transcription. 357 Overnight cultures of E. coli O127 harbouring pSVN111 (pNDM220::hipB) in combination with the empty 358 pBAD33 vector, pSVN211 (pBAD33::hipT ${ }^{\mathrm{D} 210 \mathrm{Q}}$, "hipT $\left(\mathrm{S}^{57} \mathrm{IS}^{59}, w t\right)$ ), pSVN212 (pBAD33::hipT T57D+D210Q, "hipT

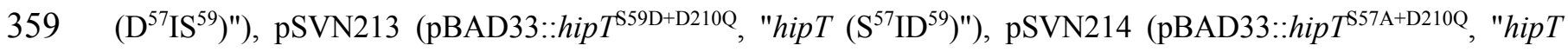

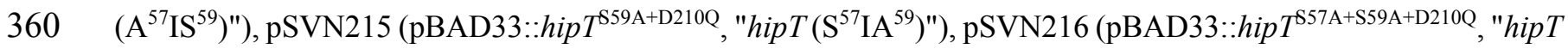

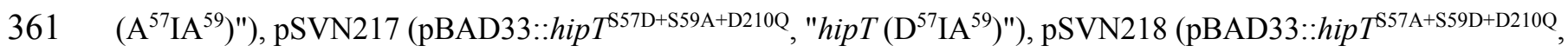

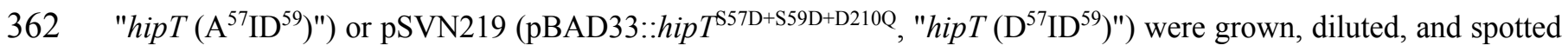
363 onto YT agar plates containing $0.2 \%$ glucose (to repress hipT variants), $0.2 \%$ arabinose (to induce hipT variants) or $3640.2 \%$ arabinose and $500 \mu \mathrm{M}$ IPTG (to induce hipB). Results are representative of two independent experiments. 
while induction of any construct containing hipB efficiently repressed transcription (Figure 5a, left, right plate, hipBST$T^{\mathrm{D} 210 \mathrm{Q}}$, hipBS, hipBT ${ }^{\mathrm{D} 210 \mathrm{Q}}$, and hipB). For hipBST and hipBT, leaky expression on repressing glucose plates was sufficient to reduce transcription. We conclude that hipB is both sufficient and required for transcriptional repression of the hipBST locus. Given that the hipBST and hipBT constructs repress transcription even in the absence of inducer, our data are also consistent with HipT (and possibly HipS) stabilising DNA interaction by HipB, as observed for many toxinantitoxin systems (Harms et al., 2018).

\section{Phosphorylation of HipT Ser57 prevents HipS binding in vivo}

In principle, the observed lack of HipS antitoxin inhibition of toxicity in the S57D phosphomimetic HipT mutants could be caused by either lack of binding of HipS or hyperactivity of the kinase. To distinguish between these two scenarios, we next tested if inactive HipT can exchange with wildtype HipT inside the HipBST complex in vivo in E. coli O127:H6. To achieve this, we constructed a series of HipT autophosphorylation variants on a background of inactive HipT $\mathrm{T}^{\mathrm{D} 210 \mathrm{Q}}$ and asked if expression would release wildtype HipT from endogenous HipBST complexes encoded by hipBST of E. coli O127:H6 and thus cause toxicity (Figure 5b, right). Both in the absence and presence of the inducer arabinose, all inactive HipT autophosphorylation variants, except those harbouring the phosphomimetic Asp57 ( ${ }^{57} \mathrm{IS}^{59}, \mathrm{D}^{57} \mathrm{IA}^{59}$, and $\mathrm{D}^{57} \mathrm{ID}^{59}$ ) were able to induce toxicity in this setup, thus suggesting that exchange can take place when Ser57 is not phosphorylated (Figure 5b, left). Of these, the $\mathrm{S}^{57} \mathrm{IS}^{59}, \mathrm{~A}^{57} \mathrm{IS}^{59}$, and $\mathrm{S}^{57} \mathrm{IA}^{59}$ variants had the strongest ability to release wildtype HipT activity while the $\mathrm{S}^{57} \mathrm{ID}^{59}, \mathrm{~A}^{57} \mathrm{IA}^{59}$ and $\mathrm{A}^{57} \mathrm{ID}^{59}$ variants activated HipT at a lower level. To exclude the possibility that expression of inactive HipT would affect the natural transcriptional repression of 
right). Due to an uncharacterised growth inhibiting effect observed upon hipBT co-expression for the inactivating kinase mutations, hip $T^{\mathrm{D} 210 \mathrm{Q}}$ (Figure 5a, left) and $h i p T^{\mathrm{D} 233 \mathrm{Q}}$ (Figure S5a), we used genetic constructs with a lower expression level of the HipT mutants for expression in combination with HipB to obtain the data in figure $5 \mathrm{c}$. Here we found similar results even with full transcriptional repression, supporting that the observed toxicity is indeed due to wildtype HipT being exchanged away from preformed endogenous HipBST complexes upon expression of inactive toxin. To confirm that the growth defects observed were in fact dependent on the hipBST system and not a secondary effect in the host, we performed a similar assay in E. coli $\mathrm{K}-12$ with the intact hipBST operon fused to lacZ encoding wildtype HipT (Figure S5b). Growth inhibition was only observed when inactive $\mathrm{Hip}^{\mathrm{D} 210 \mathrm{Q} / \mathrm{D} 233 \mathrm{Q}}$ was expressed in trans confirming that the phenotype is indeed dependent on the hipBST operon. To ensure that the low expression levels of the Hip $\mathrm{T}^{\mathrm{D} 210 \mathrm{Q}}$ autophosphorylation mutants in combination with HipB was not in themselves causing the observed growth inhibitions as seen for high expression levels (Figure 5a, left and S5a), we finally transformed the same vectors used in E. coli $\mathrm{O} 127: \mathrm{H} 6$ into E. coli K-12. None of the vectors caused growth inhibition upon induction (Figure S5f), confirming that the phenotype observed in Figure 5b must be due to the presence of the native hipBST operon. Finally, to rule out any differences in transcription of the hipBST promoter between E. coli K-12 and O127:H6, we confirmed that mRNA expression can be repressed in E. coli $\mathrm{K}-12$ when hipBST ${ }^{\mathrm{D} 210 \mathrm{Q}}$ is present in trans (Figure S5c) and that the transcriptional start sites (TSS) in the two strains are identical (Figure S5d-e). In summary, we 410 conclude that free HipT molecules are able to exchange with those inside the inhibited HipBST 411 complex, except in the S57D mutants, suggesting that phosphorylation at Ser57 controls exchange, 412 likely via control of binding of HipS. 
415 Since the protein exchange experiments suggested that the HipBST complex is dynamic in vivo, we 416 finally performed a careful analysis of the obtained small-angle X-ray scattering (SAXS) data to 417 understand the structure of the complex in solution (Figure S6a-b). We found, that the pair distance 418 distribution function indicates a maximum complex diameter of $170 \AA$, significantly larger than the 419 value calculated from the crystal structure $(140 \AA)$ suggesting some degree of structural flexibility 420 (Figure S6c-d). Moreover, fitting of the crystal structure to the solution data yielded a relatively poor 421 fit, so to accommodate internal flexibility, the HipT structure was split into three parts, roughly corresponding to the $\mathrm{N}$-terminal mini domain (1-59), the N-terminal subdomain 2 (60-169), and the core kinase domain (170-335) (Figure 2a). Following rigid body refinement against the SAXS data with soft restraints between the three parts of HipT, including HipS and HipB, and imposing two-fold symmetry, we obtained a better fit which could be further improved by allowing for partial dimerisation of the hetero-hexamer (see Material and Methods for details). In this model, the core kinase domain of HipT has a looser structure than observed in the crystal structure (root mean square deviation for $\mathrm{C} \alpha$ atoms HipT $\sim 6.5 \AA$, HipBST $\sim 7 \AA$ ) suggesting that the complex in the solution state allows for internal flexibility in the kinase. In summary, in solution structural analysis confirms that 430 the HipBST complex has a flexible structure, likely caused by structural dynamics in the HipT core 431 kinase domain, thus consistent with a model in which HipS (and perhaps HipB) can release and rebind HipT dynamically.

\section{DISCUSSION}

435 In this paper, we present a detailed structural and functional analysis of the tripartite HipBST toxinantitoxin system from enteropathogenic E. coli O127:H6. We show that most HipT kinases contain a conserved SIS motif in their Gly-rich loops, allowing for fine-tuned regulation via dual auto- 
439 positions, which may serve similar roles since Hip toxins are Ser/Thr kinases (Pereira et al., 2011).

440 Crystal structures of the HipBST complex in several, naturally occuring phosphorylation states 441 demonstrate that HipT is found in its inactive state inside the HipBST complex regardless of its 442 autophosphorylation state, consistent with a model whereby HipS, possibly aided by the DNA443 binding protein HipB, keeps the kinase in an inactive conformation and thus overrules any effects of 444 autophosphorylation. We show that this inhibition is dependent on a conserved Trp65 in HipS, which 445 couples HipS binding directly to the active site of HipT and the conformation of its Gly-rich loop. 446 Trp65 is located in a motif that differs both structurally and at the sequence level from the 447 corresponding region in the N-terminal subdomain 1 of HipA, which is otherwise very similar to 448 HipS (Gerdes et al., 2021). Together, this suggests that the molecular basis for the role of HipS as 449 antitoxin in the HipBST complex involves prevention of the Gly-rich loop from transitioning from 450 the outward, inactive state, to another conformation compatible with ATP binding (Figure 6). 


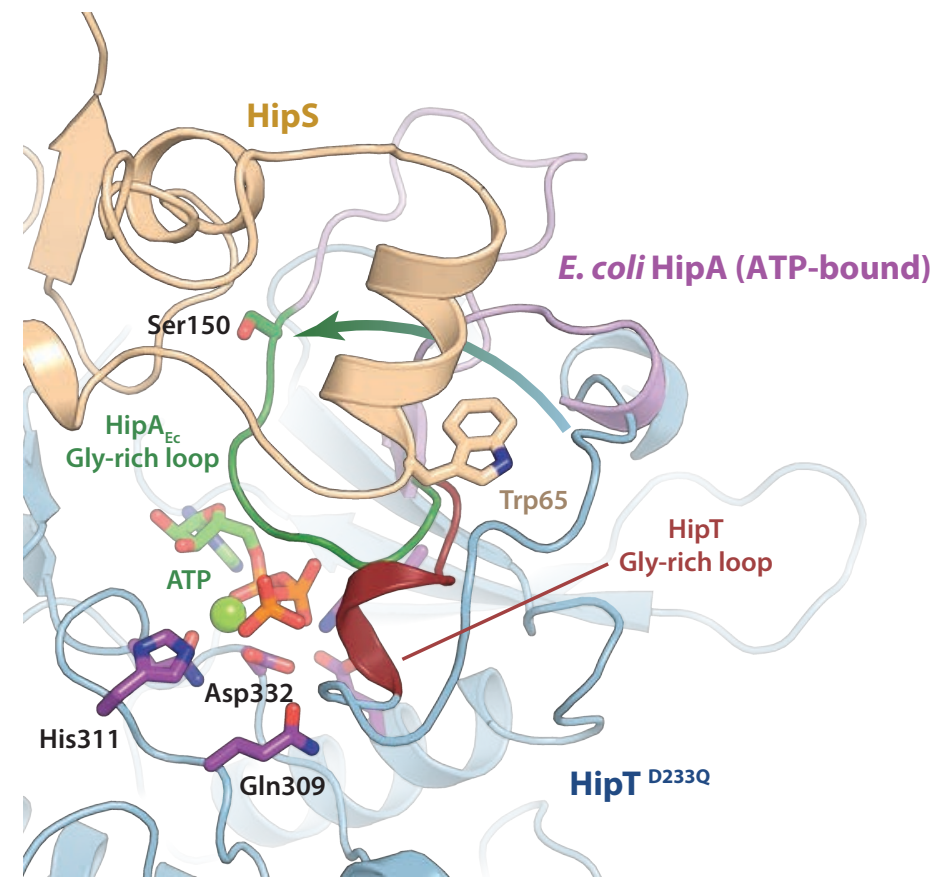

453 Figure 6. Model for the action of HipS as antitoxin in the HipBST system. Structural comparison of the positions 454 of the Gly-rich loops in HipT D233Q (blue) and E. coli K-12 HipA in its ATP-bound conformation (PDB: 3DNT, 455 green/purple) (Schumacher et al., 2009). Shown is also HipS with Trp65, which is predicted to prevent transition of 456 the Gly-rich loop from an outward-facing (HipT) to an inward-facing (HipA:ATP) conformation (shown with an 457 arrow). The active site residues of HipA as well as ATP: $\mathrm{Mg}^{2+}$ are shown with sticks on top of the HipT cartoon. 458 Ser150, which undergoes phosphorylation in HipA, is indicated as well. 
461 The size and topology of the HipT kinase with its lack of the N-terminal subdomain 1 found in HipA 462 is reminiscent of the Cyclin-dependent kinases (Cdks), which are minimal kinases that regulate the 463 eukaryotic cell cycle (Figure S7a) (Lim \& Kaldis, 2013). Intriguingly, one of these, Cdk5, also 464 regulates translation through direct phosphorylation of glutamyl-prolyl tRNA synthetase (EPRS) 465 (Arif et al., 2011). Despite differences in tertiary structure (Figure S7b), the residues involved in the 466 active site align well, suggesting common reaction mechanisms. Cyclin-dependent kinases are 467 stabilised by two hydrophobic regions, the R (regulatory) and C (catalytic) spine (Taylor \& Kornev, 468 2011). Of these, the $\mathrm{R}$ spine changes conformation upon an activating phosphorylation by flipping 469 the final active site residue into place and stabilising the kinase by forming an interaction to an 470 aspartate residue in the so-called F helix (Baugh et al., 2015). While several of these structural 471 elements are not present in HipT, the bacterial kinase does feature a conserved hydrophobic core similar to the C spine (Figure S7c-e). These regions are more extended than seen in Cdk, in particular due to the presence of two additional, conserved aromatic residues (Y138 and Y232 in HipT, Figure S7d-e). In the structure of ATP-bound HipA, the adenine base intercalates right between these and thereby connects the $\mathrm{N}$ and $\mathrm{C}$ lobes of the kinase. Interestingly, the invariant small hydrophobic residue found between the phosphor-serine positions in HipT (SIS) would connect the adenine base to the $\mathrm{N}$ lobe, suggesting that it is conserved to preserve the ATP binding. In general, it thus appears that the similarities between bacterial and eukaryotic kinases may be larger than hitherto appreciated and consequently, that the vast set of knowledge available for the eukaryotic enzymes might help us understand the Hip kinases as well. 
dynamic and allows HipT to dissociate in vitro. In this context, it is likely that the exposed conformation of the Gly-rich loop renders it particularly susceptible to phosphorylation by active

We still don't understand what controls whether HipT becomes modified at one or the other position

490 but the structures of $\mathrm{HipT}^{\mathrm{S} 57 \mathrm{~A}}$ and $\mathrm{HipT}^{\mathrm{S} 59 \mathrm{~A}}$ could suggest that subtle changes in the loop can make a 491 big difference (Figure 4a). Moreover, since Ser57/Ser59 phosphorylation was observed for both alanine single mutations in vitro, the presence of both serine residues does not appear to be important for the autophosphorylation activity of HipT. Finally, the observation that the $\mathrm{S}^{57} \mathrm{IS}^{59}, \mathrm{~A}^{57} \mathrm{IS}^{59}$, and $\mathrm{S}^{57} \mathrm{IA}^{59} \mathrm{HipT}^{\mathrm{D} 210 \mathrm{Q}}$ variants exhibit a stronger toxicity due to exchange with wildtype HipT than the $\mathrm{A}^{57} \mathrm{IA}^{59}$ variant in vivo (Figure 5b-c) indicates that the hydroxyl groups of the serine residues play a role in the binding of HipS.

Intriguingly, although the two autophosphorylation positions in HipT occupy a similar space near the $\gamma$-phosphate of ATP, they serve different roles. HipT is active in both phosphomimetic isoforms $\left(\mathrm{S}^{57} \mathrm{D}\right.$ and $\left.\mathrm{S}^{59} \mathrm{D}\right)$ while removal of the hydroxyl group on either serine by mutation to alanine $\left(\mathrm{S}^{57} \mathrm{IA}^{59}\right.$, $\mathrm{A}^{57} \mathrm{IS}^{59}$ and $\mathrm{A}^{57} \mathrm{IA}^{59}$ ) reduces its ability to cause growth inhibition. In contrast to this, we found that both $\mathrm{S}^{57} \mathrm{IA}^{59}$ and $\mathrm{A}^{57} \mathrm{IS}^{59}$ remained capable of autophosphorylation, suggesting that the presense of both of the serine residues or their phosphorylated counterparts could be important for regulating whether HipT is active on itself or its target (TrpS). Taken together, these results are consistent with 505 a model in which autophosphorylation at either position is required for target phosphorylation. 506 Surprisingly, in contrast to the $\mathrm{A}^{57} \mathrm{ID}^{59}$ mutant, the $\mathrm{D}^{57} \mathrm{IA}^{59}$ mutant was still active in inhibiting growth 507 (but with a delayed effect compared to $\mathrm{D}^{57} \mathrm{IS}^{59}, \mathrm{~S}^{57} \mathrm{ID}^{59}$, and $\mathrm{D}^{57} \mathrm{ID}^{59}$ ), suggesting that 
phosphorylation of Ser59. Finally, although the $\mathrm{D}^{57} \mathrm{ID}^{59}$ mutant is fully active in growth inhibition, this may be artificial, as dual phosphorylation has not been observed in vivo (Vang Nielsen et al., 2019). Nevertheless, the $\mathrm{D}^{57} \mathrm{ID}^{59}$ mutant gives us valuable information, as it shows that growth inhibition does not require the presence of a serine residue and that phosphorylation is likely what determines the active state. The differences in the ability of the $\mathrm{S}^{57} \mathrm{IA}^{59}$ and $\mathrm{D}^{57} \mathrm{IA}^{59}$ mutants to cause 514 growth inhibition are also striking. The $\mathrm{S}^{57} \mathrm{IA}^{59}$ mutant has a very small growth inhibitory effect, whereas $\mathrm{D}^{57} \mathrm{IA}^{59}$ has a clear effect. One explanation for this observation could be that the $\mathrm{S}^{57} \mathrm{IA}^{59}$ mutant has a slower rate of autophosphorylation compared to wildtype $\left(\mathrm{S}^{57} \mathrm{IS}^{59}\right)$ and that this would be required for HipT activity. When examining the other autophosphorylation site, Ser59, it is noteworthy, that the $\mathrm{S}^{57} \mathrm{ID}^{59}$ mutant is fully active, while the $\mathrm{A}^{57} \mathrm{IS}^{59}$ and $\mathrm{A}^{57} \mathrm{ID}^{59}$ mutants are inactive.

Another question raised by the structures is how HipS can be bound in the complex with Ser57 in its phosphorylated state if this serves to block HipS binding? In this context, it is important to note, however, that only a minor fraction of the purified complex is phosphorylated (Figure 4c), confirming that if HipT (Ser57-P) binds to HipS at all, it probably binds very poorly. It is also possible that phosphorylation occurred during purification. In either case, we believe that our data is compatible with the model for DNA binding and toxin activation for the HipT kinase toxin shown in

Figure 7. Under non-activating conditions, HipBST will be repressing the hipBST operon via binding to promoter DNA. Liberation of HipBST from DNA by an unknown mechanism will result in relaxed promoter repression and increased transcription from the operon. Free HipT could then form three populations, non-phosphorylated HipT, HipT P-Ser57, and HipT P-Ser59. Our results indicate that both phosphorylated forms are active on TrpS and as a result of this, reduce cell growth. Furthermore, 
Weak repression

Strong kinase activation

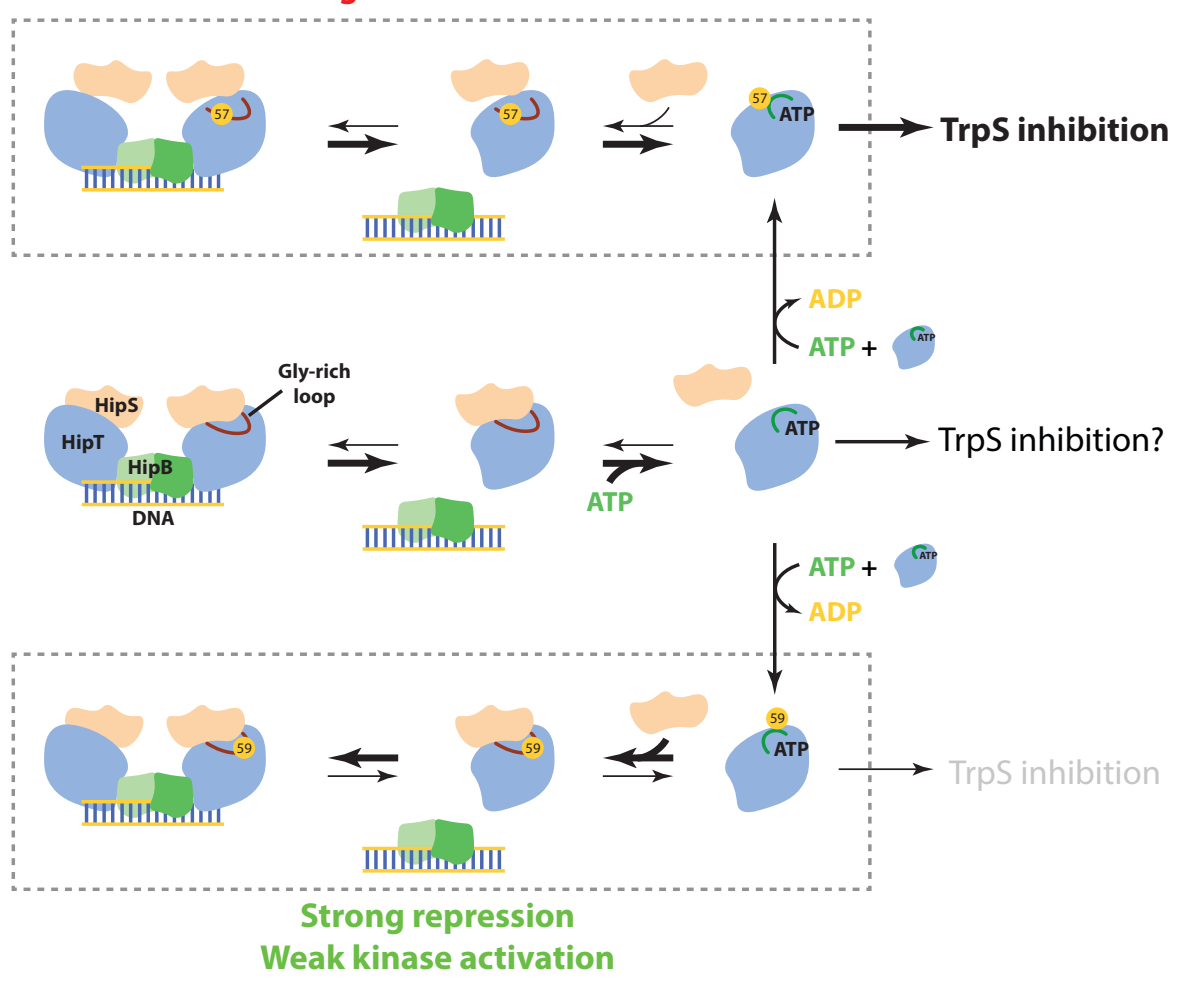

535 Figure 7. Proposed model of regulation and activation of the HipBST system. Transcription from the hipBST

536 operon (middle row, left) is repressed by binding of the HipBST hetero-hexamer in which HipT is inactive and the 537 Gly-rich loop (red) is outward-facing when HipS is bound. The dynamic HipBST complex can disassociate to 538 generate free HipT, which can phosphorylate another HipT kinase and perhaps TrpS, using ATP. 539 Autophosphorylation at Ser 57 (top box) severely reduces HipS binding, thereby prolonging the growth inhibitory 540 effect of HipT by keeping the kinase active and preventing full transcriptional repression. On the contrary, 541 autophosphorylation at Ser59 (bottom box) stabilises HipS binding and the HipBST complex, reducing the activity 542 of HipT on TrpS compared to when autophosphorylated at Ser57 and repressing transcription from the hipBST 543 operon. 
544 activity, halting the expression of new protein. On the contrary, HipT P-Ser57 would have reduced

545 HipS binding and therefore stay in its active conformation. The overall effect on growth could then 546 be a balance between these two counter-acting responses.

\section{ACKNOWLEDGEMENTS}

549 The authors are indebted to the beamline staff at P14 in EMBL Hamburg, and BioMAX in MaxIV 550 Lund for help during data collection. This project was funded by grants from the Novo Nordisk 551 Foundation (NNF18OC0030646 to D.E.B.) and Danish Natural Research Foundation's Centre of 552 Excellence for Bacterial Stress Response and Persistence (grant number DNRF120).

\section{COMPETING INTERESTS}

555 The authors confirm that there are no competing interests.

\section{AUTHOR CONTRIBUTIONS}

558 Conceptualization, K.G., M.A.S, and D.E.B.; Investigation, R.L.B., S.V.N., F. B., and J.L.; Formal 559 Analysis, R.L.B, S.V.N., F.B., and J.S.P; Writing - Original Draft, R.L.B., S.V.N., and D.E.B.; 560 Writing - Review \& Editing, R.L.B., S.V.N., J.L., J.S.P., K.G., M.A.S., and D.E.B; Visualization, 561 R.L.B., S.V.N., M.A.S., and D.E.B.; Funding Acquisition, D.E.B. and K.G.; Resources, J.S.P., K.G., 562 M.A.S. and D.E.B; Supervision, K.G., J.S.P., M.A.S., and D.E.B.

\section{MATERIALS AND METHODS}

565 Phylogenetic analysis. The previously identified set of 48 HipT orthologues (Gerdes et al., 2021) 566 was used for sequence alignment by Clustal Omega (Sievers \& Higgins, 2018) at www.ebi.ac.uk and 567 imported into Jalview (Waterhouse et al., 2009). The phylogenetic tree was visualized using iTOL 
568 (Letunic \& Bork, 2019). Reconstruction of the phylogenetic tree was accomplished using IQ-TREE 569 that uses the Maximum Likelihood approach and Ultrafast bootstrapping via the CIPRES module in 570 Genious Prime (Minh et al., 2020).

Strains and plasmids. Strains and plasmids are listed in Table S2, and DNA oligonucleotides in Table S3.

Site-directed mutagenesis. Amino acid change HipT S57A $_{\text {a }}$ was constructed by PCR mutagenesis (Table S3) as previously performed (Vang Nielsen et al., 2019). PCR product was digested with DpnI, and resulting plasmid transformed into E. coli strain DH5 $\alpha$.

Protein purification and structure determination. Expression of the E. coli O127:H6 HipBST

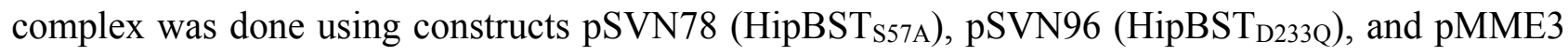
(HipBST $_{\text {S59A }}$ ) derived from pSVN78. The constructs contained the genes coding for HipB, HipS, and C-terminal hexa-histidine tagged HipT, all separated, and with optimised Shine-Dalgarno sequences

583 for large scale expression. All constructs contained an IPTG-inducible promoter and ampicillin resistance gene for selection. For each construct, $2 \mathrm{~L}$ cultures with a cell density of $\mathrm{OD}_{600}=0.6$ were induced with a final concentration of $1 \mathrm{mM}$ IPTG, and left to express $\mathrm{ON}$ at $20^{\circ} \mathrm{C}$. Cell pellets were resuspended in lysis buffer $(50 \mathrm{mM}$ Tris- $\mathrm{HCl}, \mathrm{pH}$ 7.5, $300 \mathrm{mM} \mathrm{NaCl}, 20 \mathrm{mM}$ imidazole, 5\% glycerol, $5875 \mathrm{mM}$ BME) together with $1 \mathrm{mM}$ PMSF. Cells were lysed by sonication and the cleared lysate was 588 applied to a $5 \mathrm{~mL}$ HisTrap HP column (Cytiva) equilibrated in lysis buffer, and washed in wash buffer 589 (50 mM Tris-HCl, $\mathrm{pH}$ 7.5, $300 \mathrm{mM} \mathrm{NaCl}, 40 \mathrm{mM}$ imidazole, $5 \mathrm{mM} \mathrm{BME}$ ), before eluting with elution buffer (50 mM Tris-HCl, pH 7.5, $300 \mathrm{mM} \mathrm{NaCl,} 400 \mathrm{mM}$ imidazole, $5 \mathrm{mM}$ BME). The eluate was applied directly to a $5 \mathrm{~mL}$ Heparin HF column equilibrated in 70\% Buffer A (50 mM Tris-HCl, pH 
7.5, $5 \mathrm{mM} \mathrm{BME})$ and 30\% Buffer B (50 mM Tris-HCl, pH 7.5, 1M NaCl, $5 \mathrm{mM} \mathrm{BME)} \mathrm{running} \mathrm{on}$ an ÄKTA Pure system (Cytiva). Final separation was achieved after concentrating the Heparin elution to approximately $8 \mathrm{mg} / \mathrm{mL}$ using a Vivaspin column with a $30 \mathrm{kDa}$ cutoff(Sartorius), before applying the sample to a Superdex 200 10/300 GL (Cytiva) column equilibrated in gel filtration buffer (20 $\mathrm{mM}$ Tris- $\mathrm{HCl}, \mathrm{pH} 7.5,300 \mathrm{mM} \mathrm{NaCl}, 5 \mathrm{mM}$ BME). Crystals of HipBST ${ }^{\mathrm{S} 57 \mathrm{~A}}$ grew as clusters of thin plates in a $2 \mu \mathrm{L}$ drop with a 1:1 ratio of protein to buffer, which consisted of $0.1 \mathrm{M}$ Bicine, $\mathrm{pH} 9,8 \%$ MPD and with a protein concentration of $5 \mathrm{mg} / \mathrm{mL}$, and a reservoir of $200 \mu \mathrm{L}$. Crystals of all three mutant forms were obtained by a very similar protocol. All crystals were cryoprotected in the mother liquor supplemented with 25\% MPD before freezing in liquid $\mathrm{N}_{2}$.

Data collection for HipBST ${ }^{\mathrm{S} 57 \mathrm{~A}}$ was performed at P14 in Hamburg and for HipBST ${ }^{\mathrm{S} 59 \mathrm{~A}}$ and HipBST $^{\mathrm{D} 233 \mathrm{Q}}$ at BioMAX in Lund. For HipBST ${ }^{\mathrm{S} 59 \mathrm{~A}}$ and HipBST ${ }^{\mathrm{D} 233 \mathrm{Q}}, 7,200$ images were collected with an oscillation of $0.1^{\circ}$ and a transmission of $100 \%$. The HipBST ${ }^{\text {S57A }}$ data set was collected with an oscillation of $0.1^{\circ}, 3,600$ images and a transmission of $70 \%$. All data was processed in XDS (Kabsch, 2010), using the $\mathrm{CC}^{1 / 2}$ value after scaling as indicator of the data resolution limit (Karplus \& Diederichs, 2012). The space group was confirmed using Pointless (Evans, 2006), and all structures were determined using molecular replacement with Phaser (McCoy et al., 2007) from the CCP4 suite (Winn et al., 2011). The data set for HipBST ${ }^{\mathrm{S} 57 \mathrm{~A}}$ used a heavily truncated HipA to obtain initial phases 610 for HipT, while the data set for HipBST ${ }^{\mathrm{S} 59 \mathrm{~A}}$ and HipBST ${ }^{\mathrm{D} 233 \mathrm{Q}}$ used the solved structure of HipBST 611 as a search model. Refinement was performed using Buster (Smart et al., 2012) using 1 big cycle of 61220 small cycles of refinement when building, and 5 big cycles of 100 small cycles of refinement 613 afterwards. Non-crystallographic symmetry (NCS) restraints were used during building since the 614 asymmetric unit (ASU) contained a dimer but some refinement rounds without NCS were also 615 included to allow for differences between the molecules in the ASU. Automatic water placement was 
used and water molecules with no density were removed. Model building was performed in Coot

617 (Emsley et al., 2010). All structures were validated by the MolProbity server and Rama-Z scores.

618 Final R-work/R-free for HipBST ${ }_{\mathrm{D} 233 \mathrm{Q}}, \mathrm{HipBST}_{\mathrm{S} 57 \mathrm{~A}}$, and $\operatorname{HipBST}_{\mathrm{S} 59 \mathrm{~A}}$, is $0.19 / 0.23,0.21 / 0.24$, and $619 \quad 0.20 / 0.24$, respectively.

Phos-tag gel of pre- and post-incubation with ATP. 15\% Phos-tag acrylamide gels (Wako) were cast according to the manufacturer's guidelines, except that $100 \mu \mathrm{M}$ Phos-tag acrylamide was used to ensure proper separation between phosphorylated and non-phosphorylated HipT. The nonphosphorylated HipT control was expressed from pSNN2. The gel was run at $4{ }^{\circ} \mathrm{C}$ until the loading dye reached the bottom of the gel and visualized using standard Coomassie Blue staining as for normal SDS-PAGE gels. HipBST samples were run directly on the gel orincubated with ATP for 30 minutes at room temperature in a final mixture of: $2 \mathrm{mM} \mathrm{KCl}, 3.2 \mu \mathrm{M} \mathrm{ZnSO}, 4 \mathrm{mM} \mathrm{MgCl}_{2}, 66 \mu \mathrm{M}$ ATP, $14 \mu \mathrm{L}$ gel filtration buffer, and $1 \mathrm{mg} / \mathrm{mL}$ protein sample.

Spot assays and growth curves. Cultures were grown in liquid YT medium or MOPS minimal medium $+0.2 \%$ glucose at $37^{\circ} \mathrm{C}$ shaking at $160 \mathrm{rpm}$ and solid medium used was $\mathrm{YT}$ agar plates, which were incubated at $37^{\circ} \mathrm{C}$ for approximately $16 \mathrm{~h}$. When applicable, media were supplemented with $25 \mu \mathrm{g} / \mathrm{mL}$ chloramphenicol, $30 \mu \mathrm{g} / \mathrm{mL}$ ampicillin, $25 \mu \mathrm{g} / \mathrm{mL}$ kanamycin and/or $40 \mu \mathrm{g} / \mathrm{mL} 5$ bromo-4-chloro-3-indolyl- $\beta$-D-galactopyranoside (X-gal). For spot assays, cells were grown as overnight cultures, diluted to obtain the same values of $\mathrm{OD}_{600}$, centrifuged at 5,000 rpm for $5 \mathrm{~min}$, washed in phosphate-buffered saline (PBS), and serially diluted before being spotted onto YT agar plates containing the indicated amount of inducer/repressor. Gene expression from plasmids carrying the $\mathrm{pBAD}$ promoter was induced by a final concentration of $0.2 \%$ arabinose and repressed by $0.2 \%$ 
concentration of 200 or $500 \mu \mathrm{M}$ isopropyl-D-1 thio-galactopyranoside (IPTG), as indicated. Growth experiments were done in YT medium with addition of relevant antibiotics, diluted from overnight cultures and grown exponentially for at least $4 \mathrm{~h}$ until a seemingly constant doubling time. At $\mathrm{OD}_{600}$ $\approx 0.2$, arabinose $(0.2 \%)$ was added to induce wildtype hipT or autophosphorylation mutants. After another $1.5 \mathrm{~h}$, IPTG $(200 \mu \mathrm{M})$ was added to induce hipS or hipBS. For each repetition, an independent colony from the strain was used to start separate cultures. No outliers were rejected.

Northern blot analysis. Total RNA samples were prepared using hot phenol extractions. Cultures were grown in YT medium (or MOPS minimal medium $+0.2 \%$ glucose in the case of the E. coli TB28 strain used for primer extension as described below). At indicated time points $10 \mathrm{~mL}$ were harvested into $1.25 \mathrm{~mL}$ ice-cold stop solution (5\% phenol, $\mathrm{pH} 4.3$ in absolute ethanol) and pelleted by centrifugation at $4,000 \mathrm{rpm}$ at $4{ }^{\circ} \mathrm{C}$ for $10 \mathrm{~min}$. Cell samples were resuspended in $200 \mu \mathrm{L}$ ice-cold solution I (0.3 M sucrose; 0.01 M Na-Acetate, $\mathrm{pH} 4.5)$ and mixed with $200 \mu \mathrm{L}$ solution II (2\% SDS; 0.01 M Na-Acetate, $\mathrm{pH} 4.5$ ) and $400 \mu \mathrm{L}$ phenol (pH 4.3). Samples were vortexed for $10 \mathrm{~s}$. before incubation at $65^{\circ} \mathrm{C}$ for $3 \mathrm{~min}$. followed by freezing in liquid nitrogen and centrifugation at 13,000 $\mathrm{rpm}$ at room temperature for $5 \mathrm{~min}$. The upper phase was transferred to a new tube with $400 \mu \mathrm{L}$ phenol ( $\mathrm{pH} 4.3$ ) followed by another round of the vortexing, heating, freezing and centrifugation steps. The RNA was precipitated in $1 \mathrm{~mL}$ ice-cold absolute ethanol and $50 \mu \mathrm{L}$ RNA storage buffer (3 M Na-Acetate, $\mathrm{pH} 4.7 ; 10 \mathrm{mM}$ EDTA) at $-80{ }^{\circ} \mathrm{C}$ for a minimum of $30 \mathrm{~min}$. RNA was then pelleted by centrifugation at $13,000 \mathrm{rpm}$ at $4{ }^{\circ} \mathrm{C}$ for $30 \mathrm{~min}$., washed in $70 \%$ ice-cold ethanol and air-dried at 660 room temperature. Finally, samples used for northern blotting analysis were resuspended in $20 \mu \mathrm{L}$ 661 RNA storage buffer, whereas samples used for primer extension were resuspended in $20 \mu \mathrm{L}$ nuclease662 free water. Deacylated control sample used for northern blotting analysis was prepared by mixing 4 $\mu \mathrm{L}$ of any of the total RNA samples with $6 \mu \mathrm{L} 1 \mathrm{M}$ Tris- $\mathrm{HCl}(\mathrm{pH} 9)$ and $50 \mu \mathrm{L}$ nuclease-free water 
and incubating for 2 hours at $37^{\circ} \mathrm{C}$. This was followed by the addition of $150 \mu \mathrm{L}$ ice-cold absolute ethanol and $4 \mu \mathrm{L} 5 \mathrm{M} \mathrm{NaCl}$ and the tube was incubated at $-20{ }^{\circ} \mathrm{C}$ for $15 \mathrm{~min}$. The sample was then centrifuged at $13,000 \mathrm{rpm}$ at $4{ }^{\circ} \mathrm{C}$ for $30 \mathrm{~min}$. The supernatant was discarded, and RNA pellet was air-dried at room temperature and resuspended in $4 \mu \mathrm{L}$ RNA storage buffer.

Northern analysis was used for detection and comparison of charged versus uncharged tRNA. $4 \mu \mathrm{L}$ RNA samples were mixed with $6 \mu \mathrm{L}$ tRNA loading buffer $(0.1 \mathrm{M}$ Na-succinate, $\mathrm{pH} 5 ; 8 \mathrm{M}$ urea; $0.05 \%$ bromophenol blue and $0.05 \%$ xylene cyanol) and separated on $6 \%$ polyacrylamide gels with $8 \mathrm{M}$ urea buffered in $0.1 \mathrm{M} \mathrm{Na}$-succinate ( $\mathrm{pH}$ 5) and run at $35 \mathrm{~V}$. RNA was transferred to a Hybond$\mathrm{N}+$ membrane (Amersham) at $550 \mathrm{~mA}$ constant for 1 hour in blotting buffer $(0.8 \mathrm{M}$ Tris; $40 \mathrm{mM}$ EDTA; acetic acid to $\mathrm{pH} 8.1$ ). Membranes were pre-hybridized at $42^{\circ} \mathrm{C}$ in $6 \mathrm{~mL}$ hybridization buffer (5 x SSPE; 5 x Denhardt's solution; 0.5\% SDS; $\sim 0.55 \mathrm{mg} / \mathrm{mL}$ herring sperm DNA (Sigma)) for one hour before addition of $10 \mu \mathrm{M}$ DNA oligonucleotide probe, which had been radioactively labelled with $\left[\gamma^{32} \mathrm{P}\right]-\mathrm{ATP}$, for overnight hybridization. Oligonucleotides complementary to the tRNA anticodons of interest were 5'-end labelled using T4 polynucleotide kinase (Thermo Scientific) and $40 \mu \mathrm{Ci}$ of $\left[\gamma^{32} \mathrm{P}\right]-\mathrm{ATP}(6000 \mathrm{Ci} / \mathrm{mmol})$ by incubating at $37^{\circ} \mathrm{C}$ for 2 hours. For detection of tRNA ${ }^{\operatorname{trpT}}$, tRNA ${ }^{\text {gltTUVw }}$ and tRNA ${ }^{\operatorname{argVYZQ}}$, oligonucleotides

Excess probe was removed by washing in $2 \mathrm{x}$ SSC buffer with $0.1 \%$ SDS at room temperature and radiation was visualized by phosphorimaging. Membranes were stripped for re-probing at $95{ }^{\circ} \mathrm{C}$ in stripping buffer (0.1 x SSPE; 0.5\% SDS) until no remaining radiation was detected.

Primer extension analysis. Primer extension was used to map mRNA 5' ends using radioactively 
pmol) were radioactively 5 '-end labelled with $130 \mu \mathrm{Ci}$ of $\left[\gamma^{3}{ }^{32} \mathrm{P}\right]-\mathrm{ATP}(6000 \mathrm{Ci} / \mathrm{mmol})$ using T4 polynucleotide kinase (Thermo Scientific) and incubated for $30 \mathrm{~min}$ at $37^{\circ} \mathrm{C}$ followed by inactivation for $15 \mathrm{~min}$ at $75^{\circ} \mathrm{C}$ and cooling on ice. To remove excess unincorporated $\left[\gamma_{-}{ }^{32} \mathrm{P}\right]$-ATP, the reaction was centrifuged through an illustra MicroSpin G-25 Column (Cytiva) at $750 \mathrm{~g}$ for $1 \mathrm{~min}$.

Sequencing reactions used as markers of molecular weight were generated using a purified PCR fragment amplified with primers FP43 and RP38 and 0.1 pmol from pKG127 as template DNA. The reactions were incubated with radioactively labelled primer $(0.4 \mathrm{pmol})$ and extensions were performed with DreamTaq DNA polymerase (Thermo Scientific) in the presence of either ddGTP, ddATP, ddTTP or ddCTP termination mix (Roche). PCRs were carried out and reactions were stopped by the addition of an equal volume of $2 \mathrm{x}$ formamide loading buffer $(95 \%$ formamide; $0.025 \%$ bromophenol blue and $0.025 \%$ xylene cyanol; 5 mM EDTA; $0.025 \%$ SDS).

For extension reactions, radioactively labelled oligonucleotides $(0.2 \mathrm{pmol})$ were hybridized to $10 \mu \mathrm{g}$ of total RNA by incubating at $80^{\circ} \mathrm{C}$ for $5 \mathrm{~min}$ followed by quick cooling on ice for $5 \mathrm{~min}$. Reverse transcriptions were carried out using Superscript III reverse transcriptase (Invitrogen) in 1 x FirstStrand buffer plus $10 \mathrm{mM} \mathrm{DTT}$ and $1 \mathrm{mM}$ dNTP for 1 hour at $52{ }^{\circ} \mathrm{C}$ and $47^{\circ} \mathrm{C}$ for RP35 and RP37, respectively. Reactions were stopped by the addition of an equal volume of $2 \mathrm{x}$ formamide loading urea, buffered in $1 \mathrm{x}$ TBE and run at $45 \mathrm{~W}$. Resulting gels were fixed in fixing solution (50\% ethanol; $10 \%$ acetic acid), dried and visualized by phosphor imaging. The promoter of hipBST was analysed 708 by primer extension using RP35 and RP37 for mapping of the transcriptional start site from pSVN141 709 (pGH254:: $\mathrm{P}_{\text {hipBST-hipB' }}$-lacZ) in E. coli TB28 and the native hipBST operon in E. coli O127, respectively. 
Small-angle X-ray scattering measurements and analysis. HipBST $\mathrm{S}_{\mathrm{S} 7 \mathrm{~A}}$ purified as specified in pinhole, and a VÅNTEC-2000 (Bruker AXS) microgap 2D gas proportional detector. The sample and buffer were measured in the same flow-through quartz capillary, the scattering from the buffer scattering from water. The intensity data, $I(q)$, are displayed as a function of the modulus of the scattering vector $q=4 \pi \sin (\theta) / \lambda$, where $2 \theta$ is the scattering angle and $\lambda$ is the wavelength of the forward scattering $I(0)=0.0868 \pm 0.005 \mathrm{~cm}^{-1}$. The molar mass was calculated as: $M=$ $\Delta \rho_{m}=2.00 \times 10^{10} \mathrm{~cm} \mathrm{~g}^{-1}$ is the typical excess scattering length per unit mass for proteins. The radius of gyration and forward scattering can also be obtained from the pair distance distribution function $p(r)$ determined by indirect Fourier transformation (Glatter, 1977; Pedersen et al., 1994) and the values are quite close to those from the Guinier fit: $R_{g}=53.8 \pm 0.3 \AA$ and $I(0)=0.08651 \pm$ $0.0005 \mathrm{~cm}^{-1}$, where the latter corresponds to a mass of $M=129 \mathrm{kDa}$. The maximum in a normalized Kratky plot using the values of $R_{g}$ and $I(0)$ from the Guinier fit for normalisation agrees with a 731 relatively compact structure, however, the plateau behaviour for $q R_{g}>3$ suggest some openness and potential flexibility of the structure of the complexes. The scattering of the complex from the crystal structure was directly compared to the SAXS data and a poor agreement was observed. To improve the agreement, rigid body refinement (RBR) of the structure was performed using previously 
736 first runs showed that there was some degree of oligomerisation present in the sample, since the model

737 curve is below the measured data at low $q$. Therefore, a dimer factor $S_{a g g}(q)=1+\frac{A \sin (q D)}{q D}$ (Larsen

738 et al., 2020) was multiplied with the intensity calculated for the structure. In the expression, $A$ is the

739 mass fraction of dimers of the HipBST complex and $D$ is the distance between the two copies in the

740 dimer. This approach gave good fits to the data.

742 DATA AND SOFTWARE AVAILABILITY

743 The accession numbers for the structures reported in this paper are PDB: 7AB3 (HipBST HipT 744 S57A), 7AB4 (HipBST HipT S59A), and 7AB5 (HipBST HipT D233Q). 


\section{REFERENCES}

Arif, A., Jia, J., Moodt, R. A., DiCorleto, P. E., \& Fox, P. L. (2011, Jan 25). Phosphorylation of glutamyl-prolyl tRNA synthetase by cyclin-dependent kinase 5 dictates transcript-selective translational control. Proceedings of the National Academy of Sciences of the US A, 108(4), 1415-1420. https://doi.org/10.1073/pnas.1011275108

Baugh, L., Phan, I., Begley, D. W., Clifton, M. C., Armour, B., Dranow, D. M., Taylor, B. M., Muruthi, M. M., Abendroth, J., Fairman, J. W., Fox, D., 3rd, Dieterich, S. H., Staker, B. L., Gardberg, A. S., Choi, R., Hewitt, S. N., Napuli, A. J., Myers, J., Barrett, L. K., Zhang, Y., Ferrell, M., Mundt, E., Thompkins, K., Tran, N., Lyons-Abbott, S., Abramov, A., Sekar, A., Serbzhinskiy, D., Lorimer, D., Buchko, G. W., Stacy, R., Stewart, L. J., Edwards, T. E., Van Voorhis, W. C., \& Myler, P. J. (2015, Mar). Increasing the structural coverage of tuberculosis drug targets. Tuberculosis (Edinb), 95(2), 142-148. https://doi.org/10.1016/j.tube.2014.12.003

Bordes, P., Sala, A. J., Ayala, S., Texier, P., Slama, N., Cirinesi, A. M., Guillet, V., Mourey, L., \& Genevaux, P. (2016, Nov 9). Chaperone addiction of toxin-antitoxin systems. Nat Commun, 7, 13339. https://doi.org/10.1038/ncomms13339

Brennan, R. G., \& Matthews, B. W. (1989, Feb 5). The helix-turn-helix DNA binding motif. Journal of Biological Chemistry, 264(4), 1903-1906. https://www.ncbi.nlm.nih.gov/pubmed/2644244

Emsley, P., Lohkamp, B., Scott, W. G., \& Cowtan, K. (2010, Apr). Features and development of Coot. Acta Crystallogr D Biol Crystallogr, 66(Pt 4), 486-501. https://doi.org/10.1107/S0907444910007493

Eriani, G., Delarue, M., Poch, O., Gangloff, J., \& Moras, D. (1990, Sep 13). Partition of tRNA synthetases into two classes based on mutually exclusive sets of sequence motifs. Nature, 347(6289), 203-206. https://doi.org/10.1038/347203a0

Evans, P. (2006, Jan). Scaling and assessment of data quality. Acta Crystallogr D Biol Crystallogr, 62(Pt 1), 72-82. https://doi.org/10.1107/S0907444905036693 
Evdokimov, A., Voznesensky, I., Fennell, K., Anderson, M., Smith, J. F., \& Fisher, D. A. (2009, Aug). New kinase regulation mechanism found in HipBA: a bacterial persistence switch. Acta Crystallogr D Biol Crystallogr, 65(Pt 8), 875-879. https://doi.org/10.1107/S0907444909018800

Gerdes, K., Baerentsen, R., \& Brodersen, D. E. (2021, Jun 29). Phylogeny Reveals Novel HipAHomologous Kinase Families and Toxin-Antitoxin Gene Organizations. MBio, 12(3), e0105821. https://doi.org/10.1128/mBio.01058-21

Germain, E., Castro-Roa, D., Zenkin, N., \& Gerdes, K. (2013, Oct 24). Molecular mechanism of bacterial persistence by HipA. Molecular Cell, 52(2), 248-254. https://doi.org/10.1016/j.molcel.2013.08.045

Glatter, O. (1977). New Method for Evaluation of Small-Angle Scattering Data. Journal of Applied Crystallography, 10(Oct1), 415-421. https://doi.org/Doi 10.1107/S0021889877013879

Grabe, G. J., Giorgio, R. T., Hall, A. M. J., Morgan, R. M. L., Dubois, L., Sisley, T. A., Rycroft, J. A., Hare, S. A., \& Helaine, S. (2021, Dec). Auxiliary interfaces support the evolution of specific toxin-antitoxin pairing. Nature Chemical Biology, 17(12), 1296-1304. https://doi.org/10.1038/s41589-021-00862-y

Hallez, R., Geeraerts, D., Sterckx, Y., Mine, N., Loris, R., \& Van Melderen, L. (2010, May). New toxins homologous to ParE belonging to three-component toxin-antitoxin systems in Escherichia coli O157:H7. Molecular Microbiology, 76(3), 719-732. https://doi.org/10.1111/j.1365-2958.2010.07129.x

Hanks, S. K., Quinn, A. M., \& Hunter, T. (1988, Jul 1). The protein kinase family: conserved features and deduced phylogeny of the catalytic domains. Science, 241(4861), 42-52. https://doi.org/10.1126/science.3291115

Harms, A., Brodersen, D. E., Mitarai, N., \& Gerdes, K. (2018, Jun 7). Toxins, Targets, and Triggers: An Overview of Toxin-Antitoxin Biology. Molecular Cell, 70(5), 768-784. https://doi.org/10.1016/j.molcel.2018.01.003

Haseltine, W. A., \& Block, R. (1973, May). Synthesis of guanosine tetra- and pentaphosphate requires the presence of a codon-specific, uncharged transfer ribonucleic acid in the acceptor 
site of ribosomes. Proceedings of the National Academy of Sciences of the US A, 70(5), 1564-1568. https://doi.org/10.1073/pnas.70.5.1564

Huse, M., \& Kuriyan, J. (2002, May 3). The conformational plasticity of protein kinases. Cell, 109(3), 275-282. https://doi.org/10.1016/s0092-8674(02)00741-9

Kabsch, W. (2010, Feb). Xds. Acta Crystallogr D Biol Crystallogr, 66(Pt 2), 125-132. https://doi.org/10.1107/S0907444909047337

Karplus, P. A., \& Diederichs, K. (2012, May 25). Linking crystallographic model and data quality. Science, 336(6084), 1030-1033. https://doi.org/10.1126/science.1218231

Krissinel, E., \& Henrick, K. (2007, Sep 21). Inference of macromolecular assemblies from crystalline state. Journal of Molecular Biology, 372(3), 774-797. https://doi.org/10.1016/j.jmb.2007.05.022

Larsen, A. H., Pedersen, J. S., \& Arleth, L. (2020, Aug). Assessment of structure factors for analysis of small-angle scattering data from desired or undesired aggregates. Journal of Applied Crystallography, 53(4), 991-1005. https://doi.org/10.1107/S1600576720006500

Letunic, I., \& Bork, P. (2019, Jul 2). Interactive Tree Of Life (iTOL) v4: recent updates and new developments. Nucleic Acids Research, 47(W1), W256-W259. https://doi.org/10.1093/nar/gkz239

Lim, S., \& Kaldis, P. (2013, Aug). Cdks, cyclins and CKIs: roles beyond cell cycle regulation. Development, 140(15), 3079-3093. https://doi.org/10.1242/dev.091744

Lyngsø, J., \& Pedersen, J. S. (2021). A high-flux automated laboratory small-angle X-ray scattering instrument optimized for solution scattering. Journal of Applied Crystallography, 54(1), 295-305. https://doi.org/10.1107/s1600576720016209

McCoy, A. J., Grosse-Kunstleve, R. W., Adams, P. D., Winn, M. D., Storoni, L. C., \& Read, R. J. (2007, Aug 1). Phaser crystallographic software. J Appl Crystallogr, 40(Pt 4), 658-674. https://doi.org/10.1107/S0021889807021206 
Minh, B. Q., Schmidt, H. A., Chernomor, O., Schrempf, D., Woodhams, M. D., von Haeseler, A., \& Lanfear, R. (2020, May 1). IQ-TREE 2: New Models and Efficient Methods for Phylogenetic Inference in the Genomic Era. Molecular Biology and Evolution, 37(5), 15301534. https://doi.org/10.1093/molbev/msaa015

Pacios, O., Blasco, L., Bleriot, I., Fernandez-Garcia, L., Ambroa, A., Lopez, M., Bou, G., Canton, R., Garcia-Contreras, R., Wood, T. K., \& Tomas, M. (2020, Sep 21). (p)ppGpp and Its Role in Bacterial Persistence: New Challenges. Antimicrobial Agents \& Chemotherapy, 64(10). https://doi.org/10.1128/AAC.01283-20

Pedersen, J. S., Hansen, S., \& Bauer, R. (1994). The aggregation behavior of zinc-free insulin studied by small-angle neutron scattering. European Biophysics Journal, 22(6), 379-389. https://doi.org/10.1007/BF00180159

Pereira, S. F., Goss, L., \& Dworkin, J. (2011, Mar). Eukaryote-like serine/threonine kinases and phosphatases in bacteria. Microbiology and Molecular Biology Reviews, 75(1), 192-212. https://doi.org/10.1128/MMBR.00042-10

Schumacher, M. A., Balani, P., Min, J., Chinnam, N. B., Hansen, S., Vulic, M., Lewis, K., \& Brennan, R. G. (2015, Aug 6). HipBA-promoter structures reveal the basis of heritable multidrug tolerance. Nature, 524(7563), 59-64. https://doi.org/10.1038/nature14662

Schumacher, M. A., Min, J., Link, T. M., Guan, Z., Xu, W., Ahn, Y. H., Soderblom, E. J., Kurie, J. M., Evdokimov, A., Moseley, M. A., Lewis, K., \& Brennan, R. G. (2012, Sep 27). Role of unusual P loop ejection and autophosphorylation in HipA-mediated persistence and multidrug tolerance. Cell Rep, 2(3), 518-525. https://doi.org/10.1016/j.celrep.2012.08.013

Schumacher, M. A., Piro, K. M., Xu, W., Hansen, S., Lewis, K., \& Brennan, R. G. (2009, Jan 16). Molecular mechanisms of HipA-mediated multidrug tolerance and its neutralization by HipB. Science, 323(5912), 396-401. https://doi.org/10.1126/science.1163806

Sekine, S., Nureki, O., Dubois, D. Y., Bernier, S., Chenevert, R., Lapointe, J., Vassylyev, D. G., \& Yokoyama, S. (2003, Feb 3). ATP binding by glutamyl-tRNA synthetase is switched to the productive mode by tRNA binding. EMBO Journal, 22(3), 676-688. 
Sharrock, A., Ruthe, A., Andrews, E. S. V., Arcus, V. A., \& Hicks, J. L. (2018). VapC proteins from Mycobacterium tuberculosis share ribonuclease sequence specificity but differ in regulation and toxicity. PLoS One, 13(8), e0203412. https://doi.org/10.1371/journal.pone.0203412

Sievers, F., \& Higgins, D. G. (2018, Jan). Clustal Omega for making accurate alignments of many protein sequences. Protein Science, 27(1), 135-145. https://doi.org/10.1002/pro.3290

Smart, O. S., Womack, T. O., Flensburg, C., Keller, P., Paciorek, W., Sharff, A., Vonrhein, C., \& Bricogne, G. (2012, Apr). Exploiting structure similarity in refinement: automated NCS and target-structure restraints in BUSTER. Acta Crystallogr D Biol Crystallogr, 68(Pt 4), 368380. https://doi.org/10.1107/S0907444911056058

Stancik, I. A., Sestak, M. S., Ji, B., Axelson-Fisk, M., Franjevic, D., Jers, C., Domazet-Loso, T., \& Mijakovic, I. (2018, Jan 5). Serine/Threonine Protein Kinases from Bacteria, Archaea and Eukarya Share a Common Evolutionary Origin Deeply Rooted in the Tree of Life. Journal of Molecular Biology, 430(1), 27-32. https://doi.org/10.1016/j.jmb.2017.11.004

Steiner, E. M., Lyngso, J., Guy, J. E., Bourenkov, G., Lindqvist, Y., Schneider, T. R., Pedersen, J. S., Schneider, G., \& Schnell, R. (2018, Sep). The structure of the N-terminal module of the cell wall hydrolase RipA and its role in regulating catalytic activity. Proteins, 86(9), 912923. https://doi.org/10.1002/prot.25523

Taylor, S. S., \& Kornev, A. P. (2011, Feb). Protein kinases: evolution of dynamic regulatory proteins. Trends in Biochemical Sciences, 36(2), 65-77. https://doi.org/10.1016/j.tibs.2010.09.006

Vang Nielsen, S., Turnbull, K. J., Roghanian, M., Baerentsen, R., Semanjski, M., Brodersen, D. E., Macek, B., \& Gerdes, K. (2019, Jun 18). Serine-Threonine Kinases Encoded by Split hipA Homologs Inhibit Tryptophanyl-tRNA Synthetase. MBio, 10(3). https://doi.org/10.1128/mBio.01138-19

Waterhouse, A. M., Procter, J. B., Martin, D. M., Clamp, M., \& Barton, G. J. (2009, May 1). Jalview Version 2--a multiple sequence alignment editor and analysis workbench. Bioinformatics, 25(9), 1189-1191. https://doi.org/10.1093/bioinformatics/btp033 
Wen, Y., Behiels, E., Felix, J., Elegheert, J., Vergauwen, B., Devreese, B., \& Savvides, S. N. (2014, Sep). The bacterial antitoxin HipB establishes a ternary complex with operator DNA and phosphorylated toxin HipA to regulate bacterial persistence. Nucleic Acids Research, 42(15), 10134-10147. https://doi.org/10.1093/nar/gku665

Winn, M. D., Ballard, C. C., Cowtan, K. D., Dodson, E. J., Emsley, P., Evans, P. R., Keegan, R. M., Krissinel, E. B., Leslie, A. G., McCoy, A., McNicholas, S. J., Murshudov, G. N., Pannu, N. S., Potterton, E. A., Powell, H. R., Read, R. J., Vagin, A., \& Wilson, K. S. (2011, Apr). Overview of the CCP4 suite and current developments. Acta Crystallogr D Biol Crystallogr, 67(Pt 4), 235-242. https://doi.org/10.1107/S0907444910045749

Winther, K., Tree, J. J., Tollervey, D., \& Gerdes, K. (2016, Nov 16). VapCs of Mycobacterium tuberculosis cleave RNAs essential for translation. Nucleic Acids Research, 44(20), 98609871. https://doi.org/10.1093/nar/gkw781

Winther, K. S., Roghanian, M., \& Gerdes, K. (2018, Apr 5). Activation of the Stringent Response by Loading of RelA-tRNA Complexes at the Ribosomal A-Site. Molecular Cell, 70(1), 95105 e104. https://doi.org/10.1016/j.molcel.2018.02.033

Zielenkiewicz, U., \& Ceglowski, P. (2005, Sep). The toxin-antitoxin system of the streptococcal plasmid pSM19035. Journal of Bacteriology, 187(17), 6094-6105. https://doi.org/10.1128/JB.187.17.6094-6105.2005 
TABLE S1. Crystallographic data statistics

\begin{tabular}{|c|c|c|c|}
\hline & HipBST $^{\mathrm{D} 233 \mathrm{Q}}$ & HipBST $^{\text {s57A }}$ & HipBST $^{\text {559A }}$ \\
\hline \multicolumn{4}{|l|}{ Data Collection } \\
\hline Wavelength $(\AA)$ & 1.54180 & 0.97625 & 1.54180 \\
\hline Resolution range $(\AA)$ & $49.9-2.9(3.0-2.9)^{*}$ & $46.9-2.4(2.49-2.40)^{*}$ & $50.4-3.34(3.5-3.4)^{*}$ \\
\hline Space group & $\mathrm{C} 121$ & C121 & $\mathrm{C} 121$ \\
\hline $\begin{array}{l}\text { Unit cell dimensions } \\
\mathrm{a}, \mathrm{b}, \mathrm{c},(\AA) \\
\alpha, \beta, \gamma\left({ }^{\circ}\right)\end{array}$ & $\begin{array}{l}281.66,106.47,57.75 \\
90,90.75,90\end{array}$ & $\begin{array}{l}281.68, \quad 106.07, \quad 57.56 \\
90,90.65,90\end{array}$ & $\begin{array}{l}285.26,107.15,58.45 \\
90,90.71,90\end{array}$ \\
\hline Total reflections & $74,587(7,335)$ & $625,868(25,597)$ & $41,777(4,068)$ \\
\hline Unique reflections & $37,716(3,719)$ & $66,117(6,503)$ & $22,580(2,193)$ \\
\hline Multiplicity & $2.0(2.0)$ & $9.5(3.9)$ & $1.9(1.9)$ \\
\hline Completeness (\%) & $99.8(99.3)$ & $99.9(99.4)$ & $87.71(84.84)$ \\
\hline $\mathrm{R}_{\text {meas }}(\%)$ & $0.13(1.105)$ & $0.27(0.08)$ & $0.17(1.01)$ \\
\hline $\mathrm{I} / \sigma(\mathrm{I})$ & $6.62(0.86)$ & $10.3(0.72)$ & $7.10(0.93)$ \\
\hline $\mathrm{CC}_{1 / 2}$ & $0.99(0.37)$ & $0.99(0.37)$ & $0.97(0.43)$ \\
\hline \multicolumn{4}{|l|}{ Refinement } \\
\hline Average B-factor $\left(\AA^{2}\right)$ & 98.5 & 86.1 & 119.4 \\
\hline protein & 98.9 & 86.5 & 119.9 \\
\hline ligands & - & 166.7 & 118.6 \\
\hline solvent & 80.0 & 76.7 & 78.3 \\
\hline No. of reflections & $37,706(3,719)$ & $66,112(6,504)$ & $22,570(2,193)$ \\
\hline No. of reflections (free) & $1,914(175)$ & $3,364(326)$ & $1086(86)$ \\
\hline R-work (\%) & $19.5(31.8)$ & $21.0(42.8)$ & $20.2(29.2)$ \\
\hline R-free (\%) & $22.8(34.5)$ & $23.8(46.2)$ & $24.3(35.4)$ \\
\hline \multicolumn{4}{|l|}{ Number of } \\
\hline protein (residues) & 1,018 & 1,017 & 1,023 \\
\hline solvent (atoms) & 203 & 402 & 110 \\
\hline ligand (atoms) & - & 20 & 10 \\
\hline rmsd (bonds, $\AA$ ) & 0.011 & 0.013 & 0.013 \\
\hline rmsd (angles, degrees) & 1.49 & 1.75 & 1.66 \\
\hline Rotamer outliers (\%) & 9.8 & 6.0 & 10.07 \\
\hline Clashscore & 4.4 & 2.7 & 5.8 \\
\hline \multicolumn{4}{|l|}{ Ramachandran statistics } \\
\hline favoured $(\%)$ & 95.1 & 96.0 & 95.6 \\
\hline allowed (\%) & 4.7 & 3.6 & 3.9 \\
\hline outliers (\%) & 0.2 & 0.4 & 0.5 \\
\hline \multicolumn{4}{|l|}{ Rama-Z score } \\
\hline whole & -1.63 & -0.44 & -0.90 \\
\hline helix & -1.27 & -0.40 & -0.48 \\
\hline sheet & -1.36 & 0.21 & 0.07 \\
\hline loop & -0.75 & -0.16 & -0.73 \\
\hline
\end{tabular}

${ }^{*}$ Numbers in parentheses refer to the outermost resolution shell. 
TABLE S2. Bacterial strains and plasmids.

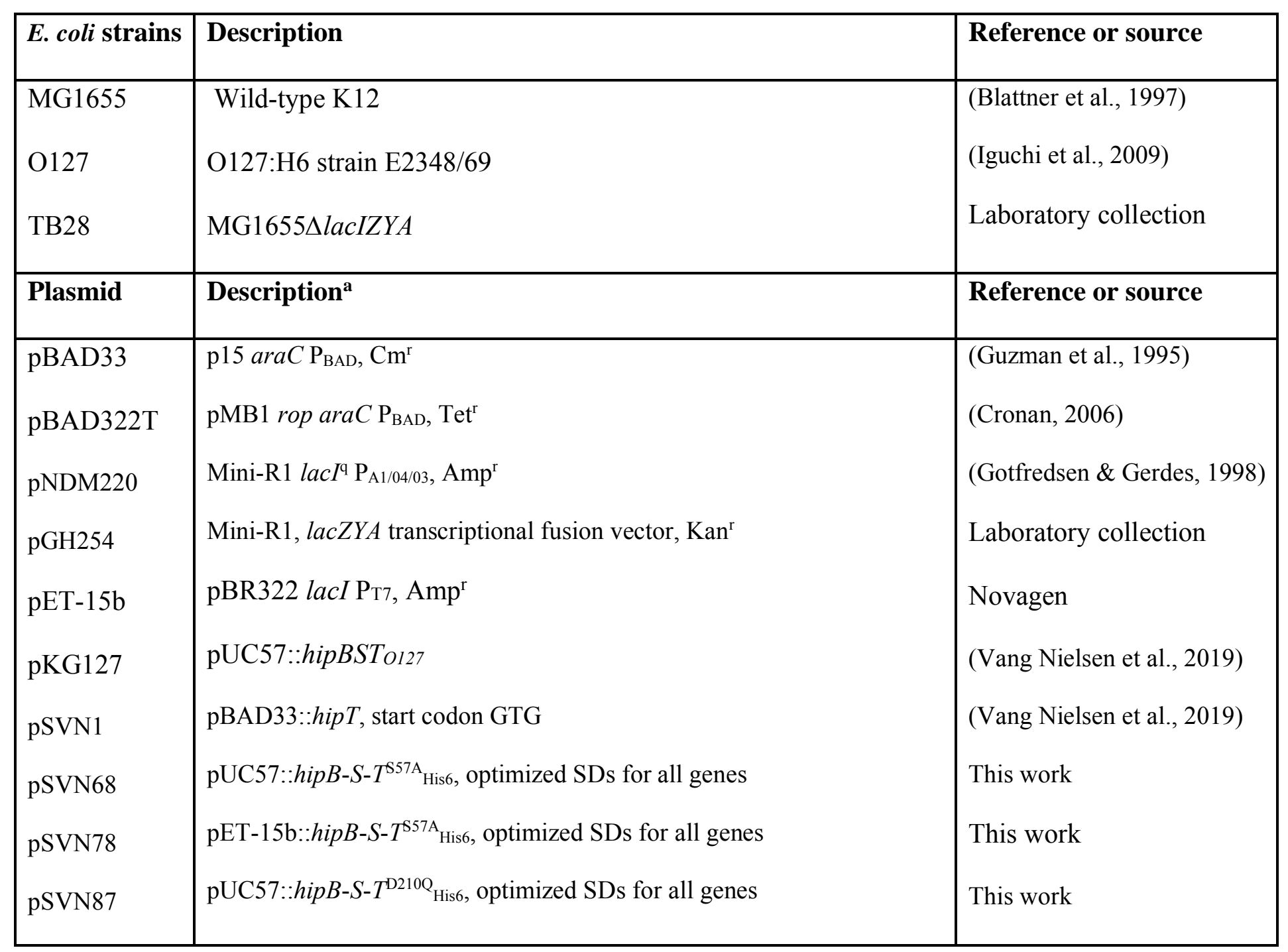




\begin{tabular}{|c|c|c|}
\hline pSVN88 & pUC57::hipB-S-T $T^{\mathrm{D} 233 \mathrm{Q}_{\mathrm{His} 6}}$, optimized SDs for all genes & This work \\
\hline pSVN96 & pET-15b::hipB-S-T $T^{\mathrm{D} 233 \mathrm{Q}_{\mathrm{His}},}$, optimized SDs for all genes & This work \\
\hline pSVN109 & pNDM220::hipS, optimized SD & (Vang Nielsen et al., 2019) \\
\hline pSVN110 & pNDM220::hipB-S, optimized SDs for both genes & (Vang Nielsen et al., 2019) \\
\hline pSVN111 & pNDM220::hipB, optimized SD & (Vang Nielsen et al., 2019) \\
\hline pSVN141 & pGH254:: $\mathrm{P}_{\text {hipBST}}$-hipB'-laZ, transcriptional $\mathrm{P}_{\text {hipBST}}$-hipB' lacZ fusion & This work \\
\hline pSVN154 & pBAD322T:: hipB-S-T $T^{\mathrm{D} 210 \mathrm{Q}}$, optimized SDs for all genes & This work \\
\hline pSVN178 & pNDM220::hip $S^{\mathrm{w} 65 \mathrm{~A}}$, optimized SD & This work \\
\hline pSVN180 & pBAD33::hipB-S-T ${ }^{\mathrm{D} 210 \mathrm{Q}}$, optimized SDs for all genes & This work \\
\hline pSVN181 & pBAD33::hipB-S-T ${ }^{\mathrm{D} 233 \mathrm{Q}}$, optimized SDs for all genes & This work \\
\hline pSVN182 & pBAD33::hipB-S, optimized SDs for both genes & This work \\
\hline pSVN184 & pBAD33::hipB-T ${ }^{\mathrm{D} 210 \mathrm{Q}}$, optimized SDs for both genes & This work \\
\hline pSVN185 & pBAD33::hipB-T ${ }^{\mathrm{D} 233 \mathrm{Q}}$, optimized SDs for both genes & This work \\
\hline pSVN187 & pBAD33::hipS-T ${ }^{\mathrm{D} 210 \mathrm{Q}}$, optimized SDs for both genes & This work \\
\hline pSVN188 & pBAD33::hipS-T $T^{\mathrm{D} 233 \mathrm{Q}}$, optimized SDs for both genes & This work \\
\hline pSVN189 & pBAD33::hipB, optimized SD & This work \\
\hline pSVN190 & pBAD33::hipS, optimized SD & This work \\
\hline
\end{tabular}




\begin{tabular}{|c|c|c|}
\hline pSVN192 & pBAD33::hipT ${ }^{\mathrm{D} 210 \mathrm{Q}}$, optimized SD & This work \\
\hline pSVN193 & pBAD33::hip ${ }^{\mathrm{D} 233 \mathrm{Q}}$, optimized SD & This work \\
\hline pSVN194 & pBAD33::hip $T^{\mathrm{S} 57 \mathrm{D}}$, start codon GTG & This work \\
\hline pSVN195 & pBAD33::hipT $T^{\mathrm{s} 59 \mathrm{D}}$, start codon GTG & This work \\
\hline pSVN198 & pGH254:: $\mathrm{P}_{\text {hipBST}}$-hipBST-lacZ, transcriptional $\mathrm{P}_{\text {hipBST }}$-hipBST lacZ fusion & This work \\
\hline pSVN199 & pBAD33::hip $T^{\mathrm{S} 57 \mathrm{~A}}$, start codon GTG & This work \\
\hline pSVN201 & pBAD33::hip $T^{\mathrm{S} 59 \mathrm{~A}}$, start codon GTG & This work \\
\hline pSVN203 & pBAD33::hip $T^{\mathrm{S} 57 \mathrm{~A}+\mathrm{S} 59 \mathrm{~A}}$, start codon GTG & This work \\
\hline pSVN205 & pBAD33::hip $T^{\mathrm{S} 57 \mathrm{D}+\mathrm{S} 59 \mathrm{~A}}$, start codon GTG & This work \\
\hline pSVN207 & pBAD33::hip $T^{\mathrm{S} 57 \mathrm{~A}+\mathrm{S} 59 \mathrm{D}}$, start codon GTG & This work \\
\hline pSVN209 & pBAD33::hip $T^{\mathrm{S} 57 \mathrm{D}+\mathrm{S} 59 \mathrm{D}}$, start codon GTG & This work \\
\hline pSVN211 & pBAD33::hipT ${ }^{\mathrm{D} 210 \mathrm{Q}}$, start codon GTG & This work \\
\hline pSVN212 & pBAD33::hip $T^{\mathrm{S} 57 \mathrm{D}+\mathrm{D} 210 \mathrm{Q}}$, start codon GTG & This work \\
\hline pSVN213 & pBAD33::hip $T^{\mathrm{5} 59 \mathrm{D}+\mathrm{D} 210 \mathrm{Q}}$, start codon GTG & This work \\
\hline pSVN214 & pBAD33::hip $T^{\mathrm{S} 57 \mathrm{~A}+\mathrm{D} 210 \mathrm{Q}}$, start codon GTG & This work \\
\hline pSVN215 & pBAD33::hip $T^{\mathrm{S} 59 \mathrm{~A}+\mathrm{D} 210 \mathrm{Q}}$, start codon GTG & This work \\
\hline pSVN216 & pBAD33::hipT $T^{\mathrm{S} 57 \mathrm{~A}+\mathrm{S} 59 \mathrm{~A}+\mathrm{D} 210 \mathrm{Q}}$, start codon GTG & This work \\
\hline
\end{tabular}




\begin{tabular}{|c|c|c|}
\hline pSVN217 & pBAD33::hipT $T^{\mathrm{S} 57 \mathrm{D}+\mathrm{S} 59 \mathrm{~A}+\mathrm{D} 210 \mathrm{Q}}$, start codon GTG & This work \\
\hline pSVN218 & pBAD33::hipT ${ }^{\mathrm{S} 57 \mathrm{~A}+\mathrm{S} 59 \mathrm{D}+\mathrm{D} 210 \mathrm{Q}}$, start codon GTG & This work \\
\hline pSVN219 & pBAD33::hipT $T^{\mathrm{S} 57 \mathrm{D}+\mathrm{S} 59 \mathrm{D}+\mathrm{D} 210 \mathrm{Q}}$, start codon GTG & This work \\
\hline pSVN220 & pBAD33::hipT $T^{\mathrm{D} 210 \mathrm{Q}}$, optimized SD & This work \\
\hline pSVN221 & pBAD33::hipT $T^{\mathrm{S} 57 \mathrm{D}+\mathrm{D} 210 \mathrm{Q}}$, optimized SD & This work \\
\hline pSVN222 & pBAD33::hipT $T^{\mathrm{S} 59 \mathrm{D}+\mathrm{D} 210 \mathrm{Q}}$, optimized SD & This work \\
\hline pSVN223 & pBAD33::hipT $T^{\mathrm{S} 57 \mathrm{~A}+\mathrm{D} 210 \mathrm{Q}}$, optimized SD & This work \\
\hline pSVN224 & pBAD33::hipT $T^{\mathrm{S} 59 \mathrm{~A}+\mathrm{D} 210 \mathrm{Q}}$, optimized SD & This work \\
\hline pSVN225 & pBAD33::hipT $T^{\mathrm{S} 57 \mathrm{~A}+\mathrm{S} 59 \mathrm{~A}+\mathrm{D} 210 \mathrm{Q}}$, optimized SD & This work \\
\hline pSVN226 & pBAD33::hip $T^{\mathrm{S} 57 \mathrm{D}+\mathrm{S} 59 \mathrm{~A}+\mathrm{D} 210 \mathrm{Q}}$, optimized SD & This work \\
\hline pSVN227 & pBAD33::hip $T^{\mathrm{S} 57 \mathrm{~A}+\mathrm{S} 59 \mathrm{D}+\mathrm{D} 210 \mathrm{Q}}$, optimized SD & This work \\
\hline pSVN228 & 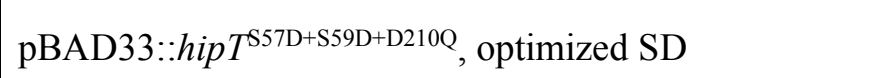 & This work \\
\hline pSNN1 & pET-15b::hipT $T_{\text {His }}^{\mathrm{S} \text {, optimized SD }}$ & This work \\
\hline pSNN2 & pET-15b::hip $T^{\mathrm{S} 57 \mathrm{~A}+\mathrm{D} 210 \mathrm{~A}}{ }_{\mathrm{His}}$, optimized SD & This work \\
\hline pMME & pET-15b::hipB-S-T $T^{\mathrm{S} 59 \mathrm{~A}}$ His6, optimized SDs for all genes & This work \\
\hline
\end{tabular}

aSD, Shine-Dalgarno sequence. 
TABLE S3. Oligonucleotides and primers.

\begin{tabular}{|c|c|}
\hline Oligonucleotide & Sequence \\
\hline FP1(GTG) & CCCCGTCGACGGATCCAAGGAGTTTTATAAGTGGCGAATTGTCGTATTCTG \\
\hline FP21 & GGGGGGTACCGGATCCAAAATAAGGAGGAAAAAAAAATGATCTGCTCAGGACCAC \\
\hline FP22 & CCCCCTCGAGGGATCCAAAATAAGGAGGAAAAAAAAATGCATCGGCGAGTGAAAG \\
\hline FP43 & CCCCGAATTCCTCTCCCGATGAGATCAGC \\
\hline FP46 & GGGGGGTACCGGATCCAAAATAAGGAGGAAAAAAAAATGCATCGGCGAGTGAAAG \\
\hline FP47 & GGGGGGTACCGGATCCAAAATAAGGAGGAAAAAAAAATGGCGAATTGTCGTATTCTG \\
\hline FP48 & GGGGGTCGACCTGCAGAAAATAAGGAGGAAAAAAAAATGGCGAATTGTCGTATTCTG \\
\hline FP49 & CCCCGAATTCCTCTCCCG ATGAGATCAGCTTTC \\
\hline RP1 & CCCCCGCATGCGAATTCGCTCACAGCAGCCCCAGACG \\
\hline RP11 & CCCCCTCGAGAAGCTTTCACAGCAGCCCCAGACG \\
\hline RP14 & GGGGGAATTCAAGCTTTTATTCCTCCCAAGGTAAAATC \\
\hline RP15 & GGGGGAATTCAAGCTTTCACTCGCCGATGCATAG \\
\hline RP32 & CCCCGGATCCTCTGCAACTCCTGGAGTTG \\
\hline RP35 & GTCCTGAGCAGATCATTAAAGAACC \\
\hline
\end{tabular}




\begin{tabular}{|c|c|}
\hline RP37 & CGATTGATAAAAGACAAAAACAAGATC \\
\hline RP38 & GTTGGTCGCCGATCGCTTTC \\
\hline RP42 & GGGGGTCGACCTGCAGTCACTCGCCGATGCATAG \\
\hline RP43 & GGGGGGTACCTCACAGCA GCCCCAGACG \\
\hline hipS W65A Fw & CAGAAGGAGCTCTGCGTCAACGCTA \\
\hline hipS W65A Rv & TGACGCAGAGCTCCTTCTGGCGC \\
\hline HipT S57D Fw & GCGTCAACAAAAAGGGATGGATATTTCCGGTT \\
\hline HipT S57D Rv & GGGCTGGTAACCGGAAATATCCATCCCTTTTT \\
\hline HipT S59D Fw & GTCAACAAAAAGGGATGAGTATTGACGGTTAC \\
\hline HipT S59D Rv & TTGGGCTGGTAACCGTCAATACTCATCCCTTT \\
\hline HipT S59A Fw & GTCAACAAAAAGGGATGAGTATTGCCGGTTAC \\
\hline HipT S59A Rv & TTGGGCTGGTAACCGGCAATACTCATCCCTTT \\
\hline HipT S57A + S59A Fw & CGTCAACAAAAAGGGATGGCTATTGCCGGTTAC \\
\hline HipT S57A + S59A Rv & TTGGGCTGGTAACCGGCAATAGCCATCCCTTTT \\
\hline HipT S57D + S59A Fw & CGTCAACAAAAAGGGATGGATATTGCCGGTTAC \\
\hline HipT S57D + S59A Rv & TTGGGCTGGTAACCGGCAATATCCATCCCTTTT \\
\hline
\end{tabular}




\begin{tabular}{|c|c|}
\hline HipT S57A + S59D Fw & CGTCAACAAAAAGGGATGGCTATTGACGGTTAC \\
\hline HipT S57A + S59D Rv & TTGGGCTGGTAACCGTCAATAGCCATCCCTTTT \\
\hline HipT S57D + S59D Fw & CGTCAACAAAAAGGGATGGATATTGACGGTTAC \\
\hline HipT S57D + S59D Rv & TTGGGCTGGTAACCGTCAATATCCATCCCTTTT \\
\hline hipX S57A Fw & AAGGGATGGCTATTTCCGGTTACCAGCC \\
\hline hipX S57A Rv & CGGAAATAGCCATCCTTTTTGTTGACG \\
\hline hipX D210Q Fw & GTAATAACCAGATGCATTTACGAAACTTTGG \\
\hline hipX D210Q Rv & AAATGCATCTGGTTATTACCCAGCAACCAGG \\
\hline hipX D233Q Fw & CGGTGTATCAGTTTGTTTCTGTCGCTCCC \\
\hline hipX D233Q Rv & GAAACAAACTGATACACCGGCGCTAACG \\
\hline hipBS del Fw & ACGACAATTCGCCATTTTTTTTTCCTCCTTATTTTTCTAGAGGG \\
\hline hipBS del Rv & TTCСССТСТAGAAAAATAAGGAGGAAAAAAAAATGGCGAAT \\
\hline hipT D210A Fw & TAAATGCATCGCGTTATTACCCAGCAACAA \\
\hline hipT D210A Rv & CTGGGTAATAACGCGATGCATTTACGAAACTTT \\
\hline hipT S57S59A Fw & GGGATGAGTATTGCCGGTTACCAGCCCAAATTGCAA \\
\hline hipT S57S59A Rv & GTAACCGGCAATACTCATCCCTTTTTGTTGACGCGG \\
\hline
\end{tabular}




\section{SUPPLEMENTARY METHODS}

2 Construction of plasmids. Construction of plasmids is summarised below.

3 pSVN68. The mutation in hipB-S-T $T^{\mathrm{S} 57 \mathrm{~A}} \mathrm{His} 6$ with optimized SDs for all three genes was created

4 using pSVN61 (Vang Nielsen et al., 2019) and primers hipX S57A Fw and hipX S57A Rv in

5 a site-directed plasmid mutagenesis PCR. Eight reactions were carried out at different

6 temperatures with a gradient PCR. The samples were pooled and digested with DpnI to digest

7 the parental plasmid before being transformed into E. coli $\mathrm{DH} 5 \alpha$.

pSVN78. hipB-S- $T^{\mathrm{S} 57 \mathrm{~A}}$ His6 with optimized $\mathrm{SDs}$ for all three genes was sub-cloned from pSVN68 by digesting with XbaI and XhoI, purifying the DNA fragment and ligating into pET$15 b$.

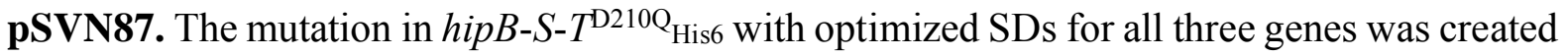
using pSVN61 (Vang Nielsen et al., 2019) and primers hipX D210Q Fw and hipX D210Q Rv in a site-directed plasmid mutagenesis PCR. Eight reactions were carried out at different temperatures with a gradient PCR. The samples were pooled and digested with DpnI to digest the parental plasmid before being transformed into E. coli $\mathrm{DH} 5 \alpha$.

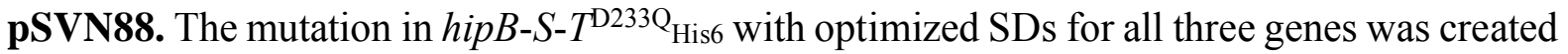
using pSVN61 (Vang Nielsen et al., 2019) and primers hipX D233Q Fw and hipX D233Q Rv in a site-directed plasmid mutagenesis PCR. Eight reactions were carried out at different temperatures with a gradient PCR. The samples were pooled and digested with DpnI to digest the parental plasmid before being transformed into E. coli $\mathrm{DH} 5 \alpha$. pSVN96. hipB-S-T $T^{\mathrm{D} 233 \mathrm{Q}_{\mathrm{His}}}$ with optimized SDs for all three genes was sub-cloned from pSVN88 by digesting with XbaI and XhoI, purifying the DNA fragment and ligating into pET$15 b$. 


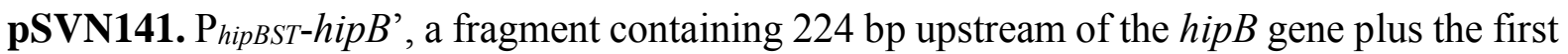
73 bp of the hipB gene was amplified from pKG127 using primers FP43 and RP32. The resulting PCR product was digested with EcoRI and BamHI and ligated into pGH254.

pSVN154. hipB-S-T $T^{\mathrm{D} 210 \mathrm{Q}}$ with optimized SDs for all three genes was amplified from pSVN87 using primers FP21 and RP11. The resulting PCR product was digested with KpnI and HindIII and ligated into pBAD322T.

pSVN178. hipS $S^{\mathrm{W} 65 \mathrm{~A}}$ was created by a two-step PCR reaction. Two fragments were amplified from pSVN109 using primers FP22 and hipS W65A Rv in one reaction and hipS W65A Fw and RP14 in the other. The resulting two PCR products were joined by a second round of PCR using both fragments as template DNA and primers FP22 and RP14. The final PCR product was digested with XhoI and EcoRI and ligated into pNDM220.

pSVN180. hipB-S-T $T^{\mathrm{D} 210 \mathrm{Q}}$ with optimized SDs for all three genes was amplified from pSVN87 using primers FP21 and RP11. The resulting PCR product was digested with KpnI and HindIII and ligated into pBAD33.

pSVN181. hipB-S-T $T^{\mathrm{D} 23 \mathrm{Q}}$ with optimized SDs for all three genes was amplified from pSVN88 using primers FP21 and RP11. The resulting PCR product was digested with KpnI and HindIII and ligated into pBAD33.

pSVN182. hip $B-S$ with optimized SDs for both genes was amplified from pSVN87 using primers FP21 and RP14. The resulting PCR product was digested with KpnI and HindIII and ligated into pBAD33.

pSVN184. hipB- $T^{\mathrm{D} 210 \mathrm{Q}}$ with optimized SDs for both genes was created by amplifying two PCR products from pSVN87: hipB using primers FP21 and RP42 and hip $T^{\mathrm{D} 210 \mathrm{Q}}$ using primers FP48 and RP11. The hipB fragment was digested with KpnI and PstI, while the hipT ${ }^{\mathrm{D} 233 \mathrm{Q}}$ fragment was digested with PstI and HindIII. The two digested fragments were then ligated into pBAD33 using the KpnI and HindIII restriction sites. 
pSVN185. hipB- $T^{\mathrm{D} 233 \mathrm{Q}}$ with optimized SDs for both genes was created by amplifying two PCR products from pSVN88: hipB using primers FP21 and RP42 and hip $T^{\mathrm{D} 233 \mathrm{Q}}$ using primers FP48 and RP11. The hipB fragment was digested with KpnI and PstI, while the hip ${ }^{\mathrm{D} 233 \mathrm{Q}}$ fragment was digested with PstI and HindIII. The two digested fragments were then ligated into pBAD33 using the KpnI and HindIII restriction sites.

pSVN187. hipS-T ${ }^{\mathrm{D} 210 \mathrm{Q}}$ with optimized SDs for both genes was amplified from pSVN88 using primers FP46 and RP11. The resulting PCR product was digested with KpnI and HindIII and ligated into $\mathrm{pBAD} 33$.

pSVN188. hipS-T $T^{\mathrm{D} 233 \mathrm{Q}}$ with optimized SDs for both genes was amplified from pSVN87 using primers FP46 and RP11. The resulting PCR product was digested with KpnI and HindIII and ligated into pBAD33.

pSVN189. hipB with optimized SD was amplified from pSVN87 using primers FP21 and

RP15. The resulting PCR product was digested with KpnI and HindIII and ligated into pBAD33.

pSVN190. hipS with optimized SD was amplified from pSVN87 using primers FP46 and RP14. The resulting PCR product was digested with KpnI and HindIII and ligated into pBAD33.

pSVN192. hip $T^{\mathrm{D} 210 \mathrm{Q}}$ with optimized SD was amplified from pSVN87 using primers FP47 and RP11. The resulting PCR product was digested with KpnI and HindIII and ligated into pBAD33.

pSVN193. hip $T^{\mathrm{D} 233 \mathrm{Q}}$ with optimized SD was amplified from pSVN88 using primers FP47 and RP11. The resulting PCR product was digested with KpnI and HindIII and ligated into pBAD33.

pSVN194. hip $T^{\mathrm{S} 57 \mathrm{D}}$ with start codon GTG was created by a two-step PCR reaction. Two fragments were amplified from pKG127 using primers FP1(GTG) and HipT S57D Rv in one 
reaction and HipT S57D Fw and RP1 in the other. The resulting two PCR products were joined by a second round of PCR using both fragments as template DNA and primers FP1(GTG) and RP1. The final PCR product was digested with SalI and SphI and ligated into pBAD33. pSVN195. hip $T^{\mathrm{S} 59 \mathrm{D}}$ with start codon GTG was created by a two-step PCR reaction. Two fragments were amplified from pKG127 using primers FP1(GTG) and HipT S59D Rv in one reaction and HipT S59D Fw and RP1 in the other. The resulting two PCR products were joined by a second round of PCR using both fragments as template DNA and primers FP1(GTG) and RP1. The final PCR product was digested with SalI and SphI and ligated into pBAD33.

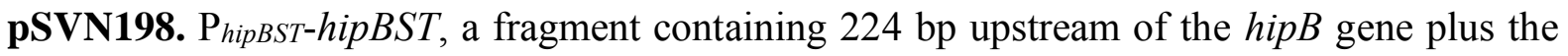
native hipBST operon was amplified from pKG127 using primers FP49 and RP43. The resulting PCR product was digested with EcoRI and KpnI and ligated into pGH254.

pSVN199. hip $T^{\mathrm{S} 57 \mathrm{~A}}$ with start codon GTG was amplified from pSVN78 using primers FP1(GTG) and RP1. The resulting PCR product was digested with SalI and SphI and ligated into pBAD33.

pSVN201. hip $T^{\mathrm{S} 59 \mathrm{~A}}$ with start codon GTG was created by a two-step PCR reaction. Two fragments were amplified from pKG127 using primers FP1(GTG) and HipT S59A Rv in one reaction and HipT S59A Fw and RP1 in the other. The resulting two PCR products were joined by a second round of PCR using both fragments as template DNA and primers FP1(GTG) and RP1. The final PCR product was digested with SalI and SphI and ligated into pBAD33. pSVN203. hip $T^{\mathrm{S} 57 \mathrm{~A}+\mathrm{S} 59 \mathrm{~A}}$ with start codon GTG was created by a two-step PCR reaction. Two 94 fragments were amplified from pKG127 using primers FP1(GTG) and HipT S57A + S59A Rv in one reaction and HipT S57A + S59A Fw and RP1 in the other. The resulting two PCR products were joined by a second round of PCR using both fragments as template DNA and 97 primers FP1(GTG) and RP1. The final PCR product was digested with SalI and SphI and 98 ligated into $\mathrm{pBAD} 33$. 
pSVN205. hip $T^{\mathrm{S} 57 \mathrm{D}+\mathrm{S} 59 \mathrm{~A}}$ with start codon GTG was created by a two-step PCR reaction. Two

100 fragments were amplified from pKG127 using primers FP1(GTG) and HipT S57D + S59A Rv

101 in one reaction and HipT S57D + S59A Fw and RP1 in the other. The resulting two PCR

102 products were joined by a second round of PCR using both fragments as template DNA and

103 primers FP1(GTG) and RP1. The final PCR product was digested with Sall and SphI and 104 ligated into pBAD33.

pSVN207. hip $T^{\mathrm{S} 57 \mathrm{~A}+\mathrm{S} 59 \mathrm{D}}$ with start codon GTG was created by a two-step PCR reaction. Two fragments were amplified from pKG127 using primers FP1(GTG) and HipT S57A + S59D Rv

107 in one reaction and HipT S57A + S59D Fw and RP1 in the other. The resulting two PCR

108 products were joined by a second round of PCR using both fragments as template DNA and 109 primers FP1(GTG) and RP1. The final PCR product was digested with Sall and SphI and 110 ligated into pBAD33.

111 pSVN209. hip ${ }^{\text {S57D+S59D }}$ with start codon GTG was created by a two-step PCR reaction. Two

112 fragments were amplified from pKG127 using primers FP1(GTG) and HipT S57D + S59D Rv

113 in one reaction and HipT S57D + S59D Fw and RP1 in the other. The resulting two PCR

114 products were joined by a second round of PCR using both fragments as template DNA and 115 primers FP1(GTG) and RP1. The final PCR product was digested with Sall and SphI and 116 ligated into pBAD33.

117 pSVN211. hipT ${ }^{\mathrm{D} 210 \mathrm{Q}}$ with start codon GTG was created by a two-step PCR reaction. Two 118 fragments were amplified from pSVN1 using primers FP1(GTG) and hipX D210Q Rv in one 119 reaction and hipX D210Q Fw and RP1 in the other. The resulting two PCR products were 120 joined by a second round of PCR using both fragments as template DNA and primers 121 FP1(GTG) and RP1. The final PCR product was digested with SalI and SphI and ligated into 122 pBAD33. 
pSVN212. hip $T^{\mathrm{S} 57 \mathrm{D}+\mathrm{D} 210 \mathrm{Q}}$ with start codon GTG was created by a two-step PCR reaction. Two

124 fragments were amplified from pSVN194 using primers FP1(GTG) and hipX D210Q Rv in one reaction and hipX D210Q Fw and RP1 in the other. The resulting two PCR products were

126 joined by a second round of PCR using both fragments as template DNA and primers

127 FP1(GTG) and RP1. The final PCR product was digested with Sall and SphI and ligated into 128 pBAD33.

pSVN213. hip $T^{\mathrm{S} 59 \mathrm{D}+\mathrm{D} 210 \mathrm{Q}}$ with start codon GTG was created by a two-step PCR reaction. Two

130 fragments were amplified from pSVN195 using primers FP1(GTG) and hipX D210Q Rv in

131 one reaction and hipX D210Q Fw and RP1 in the other. The resulting two PCR products were

132 joined by a second round of PCR using both fragments as template DNA and primers

133 FP1(GTG) and RP1. The final PCR product was digested with SalI and SphI and ligated into 134 pBAD33.

pSVN214. hip $T^{\mathrm{S} 57 \mathrm{~A}+\mathrm{D} 210 \mathrm{Q}}$ with start codon GTG was created by a two-step PCR reaction. Two

136 fragments were amplified from pSVN199 using primers FP1(GTG) and hipX D210Q Rv in

137 one reaction and hipX D210Q Fw and RP1 in the other. The resulting two PCR products were

138 joined by a second round of PCR using both fragments as template DNA and primers 139 FP1(GTG) and RP1. The final PCR product was digested with SalI and SphI and ligated into 140 pBAD33.

141 pSVN215. hip $T^{\mathrm{S} 59 \mathrm{~A}+\mathrm{D} 210 \mathrm{Q}}$ with start codon GTG was created by a two-step PCR reaction. Two 142 fragments were amplified from pSVN201 using primers FP1(GTG) and hipX D210Q Rv in 143 one reaction and hipX D210Q Fw and RP1 in the other. The resulting two PCR products were 144 joined by a second round of PCR using both fragments as template DNA and primers 145 FP1(GTG) and RP1. The final PCR product was digested with SalI and SphI and ligated into 146 pBAD33. 
pSVN216. hip $^{\mathrm{S} 57 \mathrm{~A}+\mathrm{S} 59 \mathrm{~A}+\mathrm{D} 210 \mathrm{Q}}$ with start codon GTG was created by a two-step PCR reaction.

148 Two fragments were amplified from pSVN203 using primers FP1(GTG) and hipX D210Q Rv

149 in one reaction and hipX D210Q Fw and RP1 in the other. The resulting two PCR products

150 were joined by a second round of PCR using both fragments as template DNA and primers

151 FP1(GTG) and RP1. The final PCR product was digested with SalI and SphI and ligated into

152 pBAD33.

pSVN217. hip $T^{\mathrm{S} 57 \mathrm{D}+\mathrm{S} 59 \mathrm{~A}+\mathrm{D} 210 \mathrm{Q}}$ with start codon GTG was created by a two-step PCR reaction.

154 Two fragments were amplified from pSVN205 using primers FP1(GTG) and hipX D210Q Rv

155 in one reaction and hipX D210Q Fw and RP1 in the other. The resulting two PCR products

156 were joined by a second round of PCR using both fragments as template DNA and primers

157 FP1(GTG) and RP1. The final PCR product was digested with SalI and SphI and ligated into pBAD33.

pSVN218. hip $T^{\mathrm{S} 57 \mathrm{~A}+\mathrm{S} 59 \mathrm{D}+\mathrm{D} 210 \mathrm{Q}}$ with start codon GTG was created by a two-step PCR reaction.

160 Two fragments were amplified from pSVN207 using primers FP1(GTG) and hipX D210Q Rv

161 in one reaction and hipX D210Q Fw and RP1 in the other. The resulting two PCR products were joined by a second round of PCR using both fragments as template DNA and primers FP1(GTG) and RP1. The final PCR product was digested with SalI and SphI and ligated into pBAD33.

pSVN219. hip $^{\mathrm{S} 57 \mathrm{D}+\mathrm{S} 59 \mathrm{D}+\mathrm{D} 210 \mathrm{Q}}$ with start codon GTG was created by a two-step PCR reaction.

166 Two fragments were amplified from pSVN209 using primers FP1(GTG) and hipX D210Q Rv

167 in one reaction and hipX D210Q Fw and RP1 in the other. The resulting two PCR products 168 were joined by a second round of PCR using both fragments as template DNA and primers 169 FP1(GTG) and RP1. The final PCR product was digested with SalI and SphI and ligated into pBAD33. 
pSVN220. hip $T^{\mathrm{D} 210 \mathrm{Q}}$ with optimized SD was amplified from pSVN211 using primers FP48 and RP11. The resulting PCR product was digested with PstI and HindIII and ligated into pBAD33.

pSVN221. hip $T^{\mathrm{S} 57 \mathrm{D}+\mathrm{D} 210 \mathrm{Q}}$ with optimized SD was amplified from pSVN212 using primers FP48 and RP11. The resulting PCR product was digested with PstI and HindIII and ligated into pBAD33.

pSVN222. hip $^{\mathrm{S} 59 \mathrm{D}+\mathrm{D} 210 \mathrm{Q}}$ with optimized SD was amplified from pSVN213 using primers FP48 and RP11. The resulting PCR product was digested with PstI and HindIII and ligated into pBAD33.

180 pSVN223. hip $^{\mathrm{S} 57 \mathrm{~A}+\mathrm{D} 210 \mathrm{Q}}$ with optimized SD was amplified from pSVN214 using primers

181 FP48 and RP11. The resulting PCR product was digested with PstI and HindIII and ligated into pBAD33.

pSVN224. hip $^{\mathrm{S} 59 \mathrm{~A}+\mathrm{D} 210 \mathrm{Q}}$ with optimized SD was amplified from pSVN215 using primers FP48 and RP11. The resulting PCR product was digested with PstI and HindIII and ligated into pBAD33.

pSVN225. hip $^{\mathrm{S} 57 \mathrm{~A}+\mathrm{S} 59 \mathrm{~A}+\mathrm{D} 210 \mathrm{Q}}$ with optimized SD was amplified from $\mathrm{pSVN} 216$ using primers FP48 and RP11. The resulting PCR product was digested with PstI and HindIII and ligated into pBAD33.

pSVN226. hip $T^{\mathrm{S} 57 \mathrm{D}+\mathrm{S} 59 \mathrm{~A}+\mathrm{D} 210 \mathrm{Q}}$ with optimized SD was amplified from $\mathrm{pSVN} 217$ using primers

190 FP48 and RP11. The resulting PCR product was digested with PstI and HindIII and ligated into 191 pBAD33.

pSVN227. hip $^{\mathrm{S} 57 \mathrm{~A}+\mathrm{S} 59 \mathrm{D}+\mathrm{D} 210 \mathrm{Q}}$ with optimized SD was amplified from $\mathrm{pSVN} 218$ using primers pBAD33. 
195 pSVN228. hip $^{\mathrm{S} 57 \mathrm{D}+\mathrm{S} 59 \mathrm{D}+\mathrm{D} 210 \mathrm{Q}}$ with optimized SD was amplified from pSVN219 using primers

196 FP48 and RP11. The resulting PCR product was digested with PstI and HindIII and ligated into

197 pBAD33.

198 pSNN1. hip $T^{\mathrm{S} 57 \mathrm{~A}}$ with optimized SD was amplified from pSVN78 using primers hipBS del Fw

199 and hipBS del Rv. This resulted in the deletion of HipB and HipS.

200 pSNN2. hip $T^{\mathrm{S} 57 \mathrm{~A}+\mathrm{D} 210 \mathrm{~A}}$ with optimized SD was amplified from pSNN1 using primers hipT 201 D210A Fw and hipT D210A Rv.

202 pMME3. hipBST ${ }^{\text {S59A }}$ with optimized SD was amplified from pSVN78 using primers hipT 203 S57S59A Fw and hipT S57S59A Rv to introduce the mutations A57S and S59A. 


\section{SUPPLEMENTARY FIGURE LEGENDS}

Figure S1. Sequence alignment with consensus structural elements of HipBST, HipBAEc

and HipBAso. a. Alignment of E. coli O127:H6 HipB with HipB from E. coli K-12 HipBA and HipB from S. oneidensis HipBA. Secondary structure observed in E. coli O127:H6 HipB is shown above the sequences, except for $\alpha 1^{*}$, which is missing from the crystal structures but predicted by JPred4 (Drozdetskiy et al., 2015). b. Alignment of E. coli O127:H6 HipS with the

211 N-terminal subdomain 1 from E. coli K-12 HipA and S. oneidensis HipA. The position of the the Gly-rich interacting helix ( $\alpha 3)$ of HipS is indicated. Secondary structure observed in E. coli O127:H6 HipS is shown above the sequences c. Alignment of E. coli O127:H6 HipT with the

214 main kinase domain of E. coli K-12 HipA and S. oneidensis HipA. The position of the Glyrich loop (residue 58-63) and phosphorylation sites (Ser57 and Ser59), catalytic motif (Asp210), and $\mathrm{Mg}^{2+}$-binding motif (Asp233), are indicated. Secondary structure observed in E. coli $\mathrm{O} 127: \mathrm{H} 6 \mathrm{HipT}$ is shown above the sequences.

219 Figure S2. Biophysical analysis of the HipBST complex. a. Sequence alignment of E. coli O127:H6 and selected HipS orthologues with the N-terminal subdomain 1 of E. coli K-12 HipA (bottom). Secondary structure as observed for E. coli O127:H6 HipS is indicated above the alignment. Fully conserved residues are shown on a dark orange background, partially conserved residues in orange text, and regions that interact with HipT are shown on a light grey background. Trp65 that intercalates in HipT is shown on a blue background. b. Size exclusion chromatography profile as observed for HipBST ${ }^{\mathrm{D} 233 \mathrm{Q}}$. A similar elution profile was observed for the other HipBST variants used for structural studies. Absorption at $280\left(\mathrm{~A}_{280}\right.$, blue) and $440 \mathrm{kDa}$, as indicated). c. The structure of S. oneidensis HipBA (PDB: 4PU3, HipA purple, HipB green) bound to DNA (orange backbone, blue bases) (Wen et al., 2014). 
231 Figure S3. Interactions of HipT with HipS and HipB. a. Overall fold of HipS with secondary between E. coli O127:H6 HipB (cartoon, two shades of green) and the corresponding homodimer of $S$. oneidensis HipB (PDB: 4PU3, ribbons, cyan) showing their conserved structure with similar HTH folds (Wen et al., 2014). c. Top, structural alignment of the E. coli O127:H6 HipBST complex (HipT, blue; HipS, beige; HipB, green) with S. oneidensis HipBA bound to DNA (PDB: 4PU3; HipA, purple; HipB, green) (Wen et al., 2014); Below, close-up views of conserved, basic residues on HipA that contact the DNA backbone (left) and the corresponding region in HipT (right). d. Growth curves of E. coli MG1655 grown in YT medium and harbouring empty pBAD33 vector ("pBAD33") or pSVN1 (pBAD33::hipT, "hipT"), in combination with empty pNDM220 vector ("pNDM220"), pSVN109 (pNDM220::hipS, "hipS"), or pSVN178 (pNDM220::hipS $S^{\mathrm{W} 65 \mathrm{~A}}$, "hipS ${ }^{\mathrm{W} 65 \mathrm{~A} ")}$ as indicated. At the indicated times, $0.2 \%$ arabinose was added to induce hipT (long arrow) and $200 \mu \mathrm{M}$ IPTG was added to induce hipS or hipS $S^{\mathrm{W} 65 \mathrm{~A}}$ (short arrow). The data points represent mean values of results from at least three independent experiments, and error bars show standard deviations (hidden when small).

Figure S4. HipT autophosphorylation at Ser57 and Ser59 affects function differentially. a. Active site region and Gly-rich loop (ruby) of E. coli O127:H6 HipT from the HipBST ${ }^{\text {S57A }}$ structure showing the $2 \mathrm{mFo}-\mathrm{DFc}$ difference electron density covering Ser59, contoured at 1.2 $\sigma$. The phosphate group was refined to an occupancy of 0.89 . b. Similar view from the HipBST $^{\text {S59A }}$ structure showing the $2 \mathrm{mFo-DFc}$ difference electron density covering Ser57, contoured at $1.0 \sigma$. The phosphate group was refined to an occupancy of 0.72 . c. Top, structural

254 alignment of the active sites of E. coli O127:H6 HipT S57A (blue) with E. coli K-12 HipA 
255 (HipAEc, purple) highlighting the positions of their respective phosphoserine (P-Ser) residues, as indicated; Bottom, equivalent structural alignment of the active sites of E. coli O127:H6 MG1655 strains grown in YT medium and harbouring empty pBAD33 vector, pSVN1 (pBAD33::hipT, "hipT (SIS, wt)"), or single autophosphorylation mutants pSVN194

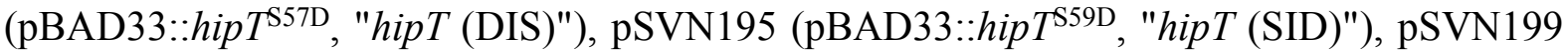
(pBAD33::hipT ${ }^{\mathrm{S} 57 \mathrm{~A}}$, "hipT (AIS)"), or pSVN201 (pBAD33::hipT ${ }^{\mathrm{S} 59 \mathrm{~A}}$, "hipT (SIA)") in combination with pSVN110 (pNDM220::hipB-S, "hipBS"). At the indicated times, $0.2 \%$ arabinose was added to induce hipT (long arrow) and $200 \mu$ Mwas added to induce hipBS (short arrow). The data points represent mean values of results from two independent experiments, and error bars show standard deviations (hidden when small). e. As in $\mathbf{d}$ but for double autophosphorylation mutants pSVN203 (pBAD33::hipt ${ }^{\mathrm{S} 57 \mathrm{~A}+\mathrm{S} 59 \mathrm{~A}}$, "hipT (AIA)"), pSVN205

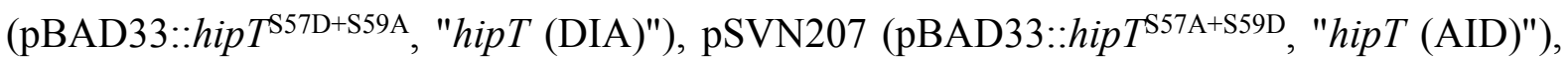

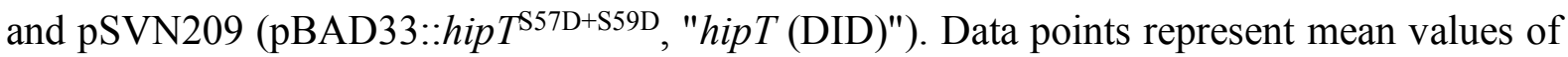
results from two independent experiments, and error bars designate standard deviations. f.

270 Northern blot analysis using probe directed against tRNA ${ }^{\text {gltTUVW }}$ (top) and tRNA ${ }^{\text {argVYZQ }}$ 271 (bottom) based on RNA extracted from E. coli MG1655 harbouring pSVN110 (pNDM220::hipB-S) in combination with pSVN1 (pBAD33::hipT, "hipT $\left.\left(\mathrm{S}^{57} \mathrm{IS}^{59}, w t\right) "\right)$, pSVN194 (pBAD33::hipT T57D, "hipT (D $\left.\left.{ }^{57} \mathrm{IS}^{59}\right) "\right)$, pSVN195 (pBAD33::hipT ${ }^{\mathrm{S} 59 \mathrm{D}}$, "hipT $\left.\left(\mathrm{S}^{57} \mathrm{ID}^{59}\right)^{\prime}\right), \quad$ pSVN209 $\quad\left(\mathrm{pBAD} 33:\right.$ hip ${ }^{\mathrm{S} 57 \mathrm{D}+\mathrm{S} 59 \mathrm{D}}, \quad$ "hipT $\left.\quad\left(\mathrm{D}^{57} \mathrm{ID}^{59}\right)^{\prime \prime}\right)$ or pSVN205

275 (pBAD33::hip $T^{\mathrm{S} 57 \mathrm{D}+\mathrm{S} 59 \mathrm{~A}}$, "hipT $\left(\mathrm{D}^{57} \mathrm{IA}^{59}\right)$ ") at indicated time points in minutes before (-) and after $(+)$ addition of $0.2 \%$ arabinose to induce wildtype hipT or autophosphorylation mutants.

277 Results from strains harbouring pSVN1 (pBAD33::hipT), pSVN194 (pBAD33::hipT ${ }^{\mathrm{S} 57 \mathrm{D})}$,

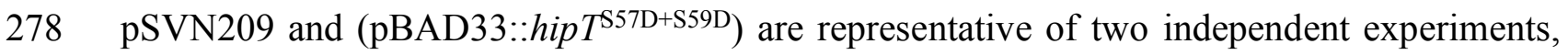


whereas results from strains harbouring pSVN195 (pBAD33::hip $T^{\mathrm{S} 59 \mathrm{D}}$ ) and pSVN205 (pBAD33::hip $\left.T^{\mathrm{S} 57 \mathrm{D}+\mathrm{S} 59 \mathrm{~A}}\right)$ are from single experiments.

Figure S5. Transcriptional regulation of the HipBST system of $E$. coli O127:H6. a.

283

Transcriptional reporter assay using a plasmid with the hipBST promoter region and a 5' fragment of the $h i p B$ gene (including 224 bp upstream of hipB plus the first 73 bp of the hipB gene, shown with a lightening symbol) transcriptionally fused to lacZ. E. coli TB28 harbouring

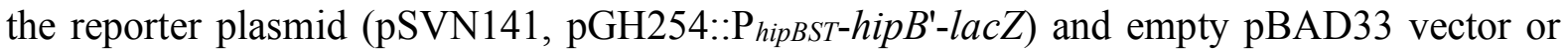
combinations of hipB, hipS, and hipT in the context of the HipT ${ }^{\mathrm{D} 233 \mathrm{Q}}$ inactive mutant; pSVN180 (pBAD33::hipB-S-T ${ }^{\mathrm{D} 233 \mathrm{Q}}$, "hipBST $\left.{ }^{\mathrm{D} 233 \mathrm{Q}}\right)$ ), pSVN182 (pBAD33::hipB-S, "hipBS"), pSVN184

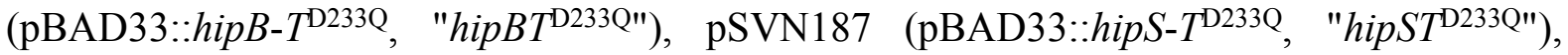
pSVN189 (pBAD33::hipB, "hipB"), pSVN190 (pBAD33::hipS, "hipS"), or pSVN192 (pBAD33::hipT $T^{\mathrm{D} 233 \mathrm{Q}}$, "hipT $\left.{ }^{\mathrm{D} 233 \mathrm{Q}}\right)$ were grown, diluted, and spotted onto YT agar plates containing $40 \mu \mathrm{g} / \mathrm{ml} \mathrm{X-gal} \mathrm{and} 0.2 \%$ glucose (to repress $h i p B / S / T^{\mathrm{D} 233 \mathrm{Q}}$ ) or $0.2 \%$ arabinose (to induce hipB/S/T $\left.{ }^{\mathrm{D} 233 \mathrm{Q}}\right)$. Results are representative of two independent experiments. b. Overexpression of inactive Hip $\mathrm{T}^{\mathrm{D} 210 \mathrm{Q}}$ and $\mathrm{HipT}^{\mathrm{D} 233 \mathrm{Q}}$ increases the level of active wildtype HipT from the hipBST operon transcriptionally fused to lacZ in $E$. coli TB28. The strain harbouring empty pGH254 vector or pSVN198 (pGH254::PhipBST-hipBST-lacZ, "hipBST") in combination with the empty pBAD33 vector, pSVN192 (pBAD33::hipT $T^{\mathrm{D} 210 \mathrm{Q}}$, "hipt ${ }^{\mathrm{D} 210 \mathrm{Q}}$ "), or

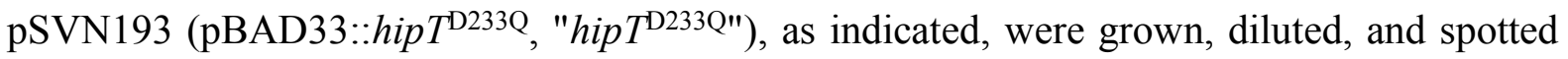
onto YT agar plates containing $40 \mu \mathrm{g} / \mathrm{ml} \mathrm{X-gal} \mathrm{without} 0.2 \%$ arabinose (to obtain only a leaky expression of hipT $T^{\mathrm{D} 210 \mathrm{Q} / \mathrm{D} 233 \mathrm{Q}}$ ), or with $0.2 \%$ arabinose (to induce hipT $\left.T^{\mathrm{D} 210 \mathrm{Q} / \mathrm{D} 233 \mathrm{Q}}\right)$. Results are from a single experiment. c. Primer extension analysis mapping the transcriptional start site in

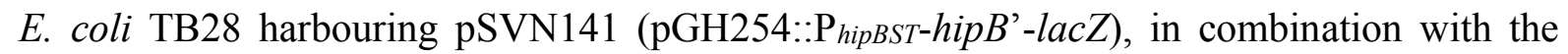
empty pBAD322T vector, or pSVN154 (pBAD322T::hipB-S-T $\left.{ }^{\mathrm{D} 210 \mathrm{Q}}\right)$, as indicated were grown 
and harvested at $\mathrm{OD}_{600} \approx 0.4$. d. Primer extension analysis mapping the transcriptional start site in E. coli $\mathrm{O} 127: \mathrm{H} 6$ harbouring pSVN192 (pBAD33::hipT ${ }^{\mathrm{D} 210 \mathrm{Q}}$ ). At $\mathrm{OD}_{600} \approx 0.4,0.2 \%$ arabinose was added (to induce hip ${ }^{\mathrm{D} 210 \mathrm{Q}}$ ), samples were taken at indicated time points and primer extension performed. e. The hipBST promoter region showing the transcriptional start site

308 (arrow) and corresponding -10 and -35 sequences (red). The start codon of the hipB gene 309 ("Met") and putative Shine-Dalgarno sequence ("SD") are indicated as well. f. Spot assays using E. coli MG1655 harbouring pSVN111 (pNDM220::hipB) in combination with the empty pBAD33 vector, pSVN211 (pBAD33::hipT ${ }^{\mathrm{D} 210 \mathrm{Q},}$ "hipT $\left.\left(\mathrm{S}^{57} \mathrm{IS}^{59}, w t\right) "\right), \quad$ SSVN212

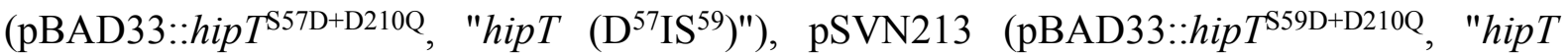
$\left.\left(\mathrm{S}^{57} \mathrm{ID}^{59}\right)^{\prime}\right), \quad$ pSVN214 $\quad\left(\right.$ pBAD33::hipT $T^{\mathrm{S} 57 \mathrm{~A}+\mathrm{D} 210 \mathrm{Q},} \quad$ "hipT $\left.\quad\left(\mathrm{A}^{57} \mathrm{IS}^{59}\right) "\right), \quad$ pSVN215 (pBAD33::hipT $T^{\mathrm{S} 59 \mathrm{~A}+\mathrm{D} 210 \mathrm{Q},}$ "hipT $\left(\mathrm{S}^{57} \mathrm{IA}^{59}\right)$ ), pSVN216 (pBAD33::hipT $T^{\mathrm{S} 57 \mathrm{~A}+\mathrm{S} 59 \mathrm{~A}+\mathrm{D} 210 \mathrm{Q}}$, "hipT $\left.\left(\mathrm{A}^{57} \mathrm{IA}^{59}\right)^{\prime \prime}\right), \quad$ pSVN217 (pBAD33::hip $T^{\mathrm{S} 57 \mathrm{D}+\mathrm{S} 59 \mathrm{~A}+\mathrm{D} 210 \mathrm{Q}}, \quad$ "hipT $\left.\quad\left(\mathrm{D}^{57} \mathrm{IA}^{59}\right)^{\prime}\right), \quad$ pSVN218

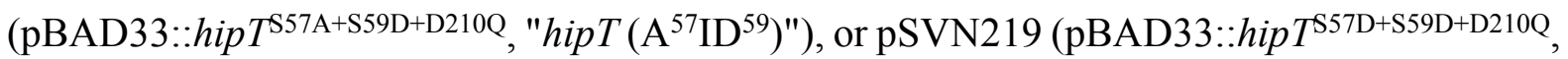
autophosphorylation mutants), $0.2 \%$ arabinose (to induce hip $T^{\mathrm{D} 210 \mathrm{Q}}$ autophosphorylation mutants) or $0.2 \%$ arabinose plus $500 \mu \mathrm{M}$ IPTG (to induce hipB). Results are from a single experiment.

Figure S6. Analysis of HipBST using small-angle x-ray scattering. a. Backgroundsubtracted and normalized SAXS data measured for HipBST ${ }^{\mathrm{S} 57 \mathrm{~A}}$ with indirect Fourier transform fit. b. Guinier plot. c. $p($ r) from indirect Fourier transform. d. Normalized Kratky plot using $I(0)$ and $R_{g}$ from the Guinier fit. 
bioRxiv preprint doi: https://doi.org/10.1101/2022.01.28.478185; this version posted January 31,2022 . The copyright holder for this preprint (which was not certified by peer review) is the author/funder, who has granted bioRxiv a license to display the preprint in perpetuity. It is made available under aCC-BY 4.0 International license.

329 HipT and human CDK5 showing the unique N-terminal mini-domain found in HipT (dashed)

330 and that the $\mathrm{N}$-terminal subdomain 2 is similar to the $\mathrm{N}$-terminal lobe of CDK 5 while the core

331 kinase domain is similar to the C-terminal lobe. Conserved features and active site residues are

332 marked with numbers and listed below. b. Structural comparison of E. coli O127:H6 HipT

333 (top) and human CDK5 (PDB: 4AU8, bottom) (Malmstrom et al., 2012) shown in rainbow

334 colours from $\mathrm{N}$ (blue) to $\mathrm{C}$ (red) terminus. $\alpha$-helices are shown as cylinders and for CDK5

335 named in accordance with classical Cdk nomenclature. The two proteins differ in tertiary fold

336 despite similar topology. c. Top, overview structure of Human CDK9 (salmon, PDB: 3BLT)

337 with the C-spine region shown as surface (cyan) (Baumli et al., 2008); below, details of the

338 active site bound to ATP with relevant residues indicated. d. Top, overview of the E. coli

339 O127:H6 HipT structure with the C/R spine region shown as surface (green); below, details of

340 the active site. e. Top, overview of the E. coli K-12 HipA structure (PDB: 3DNT) with the C/R

341 spine region shown as surface (orange) (Schumacher et al., 2009); below, details of the active

342 site bound to ATP. 


\section{REFERENCES}

345

Baumli, S., Lolli, G., Lowe, E. D., Troiani, S., Rusconi, L., Bullock, A. N., Debreczeni, J. E., Knapp, S., \& Johnson, L. N. (2008, Jul 9). The structure of P-TEFb (CDK9/cyclin T1), its complex with flavopiridol and regulation by phosphorylation. EMBO Journal, 27(13), 1907-1918. https://doi.org/10.1038/emboj.2008.121

Blattner, F. R., Plunkett, G., 3rd, Bloch, C. A., Perna, N. T., Burland, V., Riley, M., ColladoVides, J., Glasner, J. D., Rode, C. K., Mayhew, G. F., Gregor, J., Davis, N. W., Kirkpatrick, H. A., Goeden, M. A., Rose, D. J., Mau, B., \& Shao, Y. (1997, Sep 5). The complete genome sequence of Escherichia coli K-12. Science, 277(5331), 1453-1462. https://doi.org/10.1126/science.277.5331.1453

Cronan, J. E. (2006, Mar). A family of arabinose-inducible Escherichia coli expression vectors having pBR322 copy control. Plasmid, 55(2), 152-157. https://doi.org/10.1016/j.plasmid.2005.07.001

Drozdetskiy, A., Cole, C., Procter, J., \& Barton, G. J. (2015, Jul 1). JPred4: a protein secondary structure prediction server. Nucleic Acids Research, 43(W1), W389-394. https://doi.org/10.1093/nar/gkv332

Gotfredsen, M., \& Gerdes, K. (1998, Aug). The Escherichia coli relBE genes belong to a new toxin-antitoxin gene family. Molecular Microbiology, 29(4), 1065-1076. https://doi.org/10.1046/j.1365-2958.1998.00993.x

Guzman, L. M., Belin, D., Carson, M. J., \& Beckwith, J. (1995, Jul). Tight regulation, modulation, and high-level expression by vectors containing the arabinose PBAD promoter. Journal of Bacteriology, 177(14), 4121-4130. https://doi.org/10.1128/jb.177.14.4121-4130.1995

Iguchi, A., Thomson, N. R., Ogura, Y., Saunders, D., Ooka, T., Henderson, I. R., Harris, D., Asadulghani, M., Kurokawa, K., Dean, P., Kenny, B., Quail, M. A., Thurston, S., Dougan, G., Hayashi, T., Parkhill, J., \& Frankel, G. (2009, Jan). Complete genome sequence and comparative genome analysis of enteropathogenic Escherichia coli O127:H6 strain E2348/69. Journal of Bacteriology, 191(1), 347-354. https://doi.org/10.1128/JB.01238-08 
Malmstrom, J., Viklund, J., Slivo, C., Costa, A., Maudet, M., Sandelin, C., Hiller, G., Olsson, L. L., Aagaard, A., Geschwindner, S., Xue, Y., \& Vasange, M. (2012, Sep 15). Synthesis and structure-activity relationship of 4-(1,3-benzothiazol-2-yl)-thiophene-2sulfonamides as cyclin-dependent kinase $5(\operatorname{cdk} 5) / \mathrm{p} 25$ inhibitors. Bioorganic and Medicinal Chemistry

Letters, 22(18), 5919-5923. https://doi.org/10.1016/j.bmc1.2012.07.068

Schumacher, M. A., Piro, K. M., Xu, W., Hansen, S., Lewis, K., \& Brennan, R. G. (2009, Jan 16). Molecular mechanisms of HipA-mediated multidrug tolerance and its neutralization by HipB. Science, 323(5912), 396-401. https://doi.org/10.1126/science.1163806

Vang Nielsen, S., Turnbull, K. J., Roghanian, M., Baerentsen, R., Semanjski, M., Brodersen, D. E., Macek, B., \& Gerdes, K. (2019, Jun 18). Serine-Threonine Kinases Encoded by Split hipA Homologs Inhibit Tryptophanyl-tRNA Synthetase. MBio, 10(3). https://doi.org/10.1128/mBio.01138-19

Wen, Y., Behiels, E., Felix, J., Elegheert, J., Vergauwen, B., Devreese, B., \& Savvides, S. N. (2014, Sep). The bacterial antitoxin HipB establishes a ternary complex with operator DNA and phosphorylated toxin HipA to regulate bacterial persistence. Nucleic Acids Research, 42(15), 10134-10147. https://doi.org/10.1093/nar/gku665 


\section{Figure S1}

E. coli 0127:H6 HipB (HipBST) E. coli 0127:H6 HipB (HipBST) E. coli K-12 HipB (HipBA)
S. oneidensis HipB (HipBA)

\section{b}

E. coli 0127:H6 HipS (HipBST) E. coli 0127:H6 HipS (HipBST) S. oneidensis HipA (HipBA)

\section{C}

E. coli 0127:H6 Hipt (HipBST) E. coli 0127:H6 Hipt (HipBST) E. coli K-12 HipA (HipBA)

E. coli 0127:H6 HipT (HipBST) E. coli O127:H6 HipT (HipBST) E. coli K-12 HipA (HipBA)
S. oneidensis HipA (HipBA)

E. coli 0127:H6 HipT (HipBST) E. coli 0127:H6 HipT (HipBST) E. coli K-12 HipA (HipBA)
S. oneidensis HipA (HipBA)

E. coli O127:H6 HipT (HipBST) E. coli K-12 HipA (HipBA) elecelecellecelecele eceecelecece $\alpha 2$

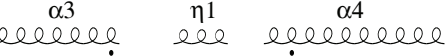

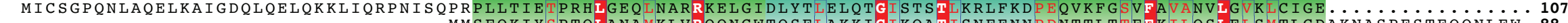

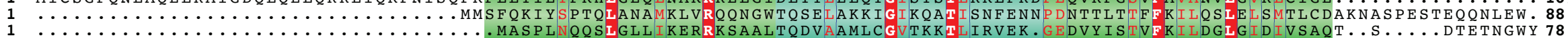

Gly-rich interacting helix

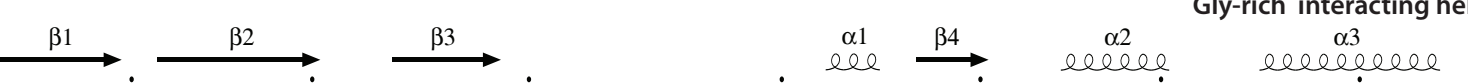$$
\text { eeeee }
$$

$\stackrel{\beta 5}{\longrightarrow}$

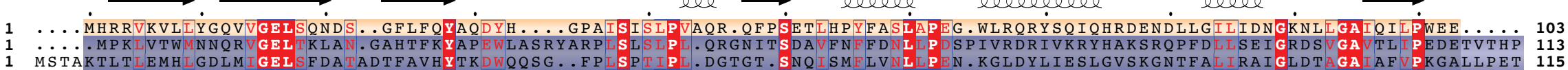

Gly-rich loop

Ser57 Ser59

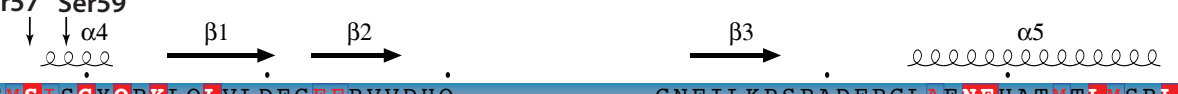

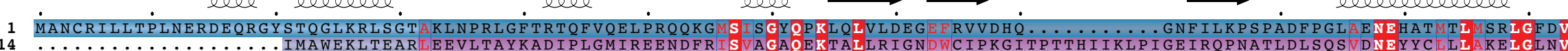

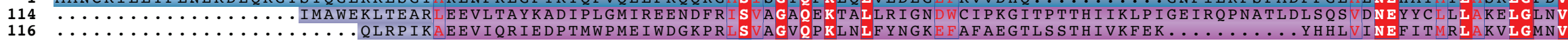

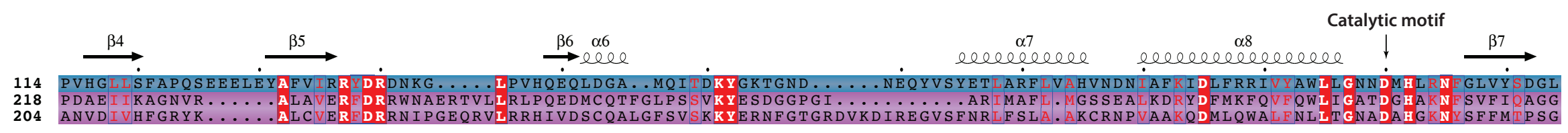

$\mathrm{Mg}^{2+}$-binding

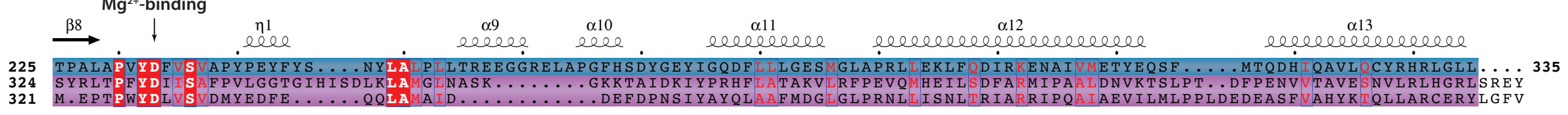

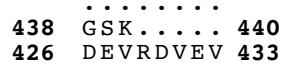


Figure S2 bioRxiv preprint doi: https://doi.org/10.1101/2022.01.28.478185; this version posted January 31, 2022. The copyright holder for this preprint (which was not certified by peer review) is the author/funder, who has granted bioRxiv a license to display the preprint in perpetuity. It is made available under aCC-BY 4.0 International license.

a

E. coli 0127:H6 HipS V. bacterium HipS B. ovatus HipS

N. bacterium HipS

Prevotella sp. Hips

B. bacterium HipS

E. coli K-12 HipA

\section{$\stackrel{\beta 1}{\longrightarrow} \frac{\beta 2}{\rightarrow} \frac{\beta 3}{\longrightarrow}$} MTSDASNRVRGRWAE IFQRGVPAGVIEEQAGGGWGFRYLEGY. . . SGPPVSI TMPVAERSFQFE $\ldots \ldots \ldots . . . . M S R S A K V Y$ I KG I YAGLITE IDRE H Y S FCY DT DYYNN POL PAVS L TMPKTOOEYT SS

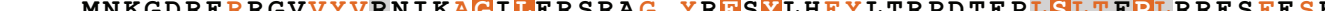
...........MKTAIVY $\ldots \ldots \ldots$ MRKAAIKIENELAGWLT QDELG. YHEVYEKSYAMK PGARPVSL TLPLRDEPYTSK

$1 \ldots . . . . . M$ MPKLVTWMNQRVGELTKLANGAHTEKYAPEWLASRYARPLSLSLPLQRGNITSD a3 elele el elelele 52 作 YLEPVFFNMTSEGDN . RIIQARNLHIDEEDDFGILIATAHTDTIGAITVKRI . . . . . . . . . KMEPYFLGL I PEGWLL. DLTMRTLKIDPENVFE LILCCCK. DCVGTTS IYPEGEEVK........ VLF P FDGLI PEGWLL. DIAEK SWKINQRDRMSLILACCK. DCIGAVSVVPEQDDKEEEN . . . Prevotella sp. Hips VLF P FDGLI PEGWLL. DIAERNWKLNPRDRMGLILVCCK. DCIGNVSVEAINEENLP...... .

E. coli K-12 HipA AVFN FTDNLLPDSPIVRDRIVKRYHAKSRQPFDLISEIGR. DSVGAVT LI PEDETVTHPIMAWEK

b

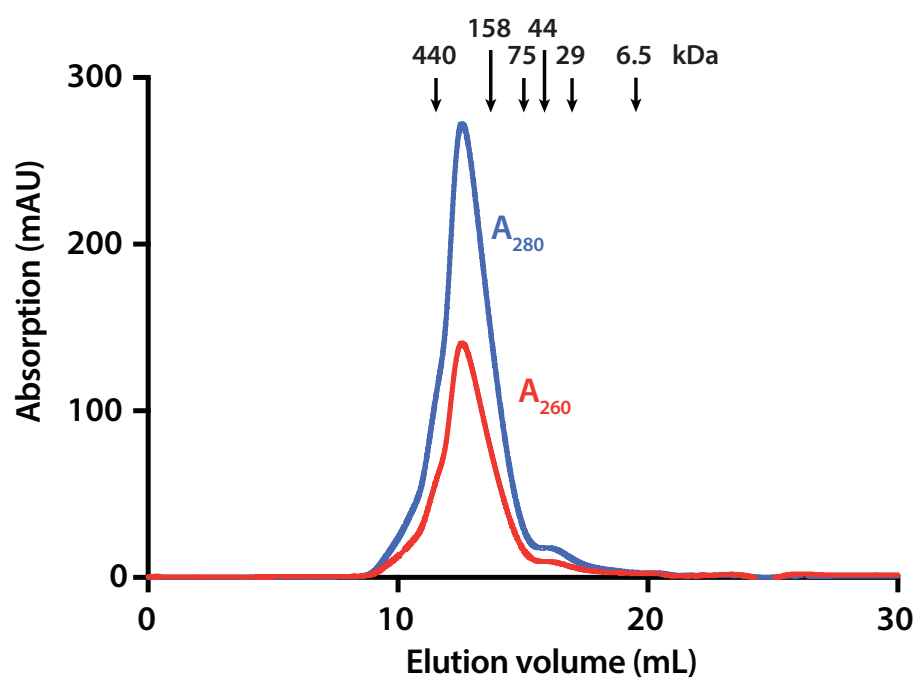

C

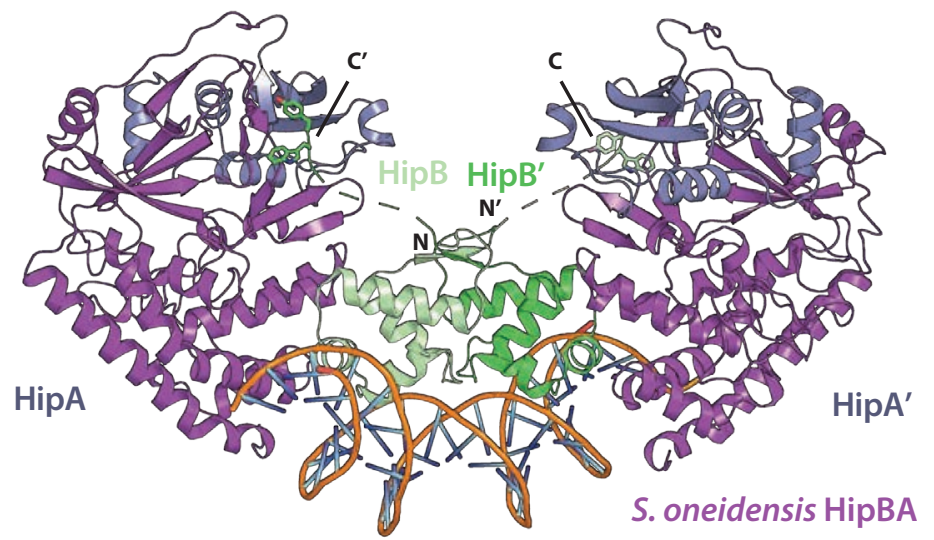


Figure S3 bioRxiv preprint doi: https://doi.org/10.1101/2022.01.28.478185; this version posted January 31, 2022. The copyright holder for this preprint (which was not certified by peer review) is the author/funder, who has granted bioRxiv a license to display the preprint in

a

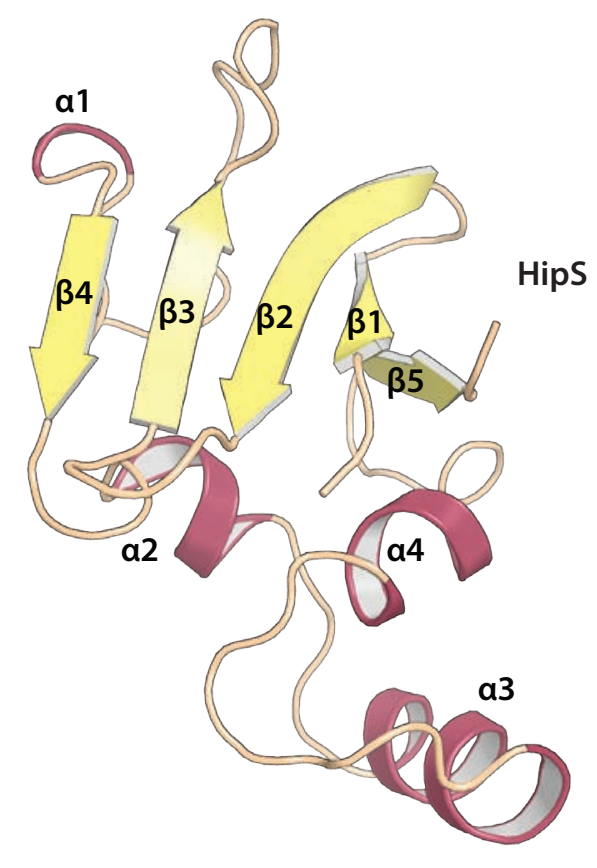

b

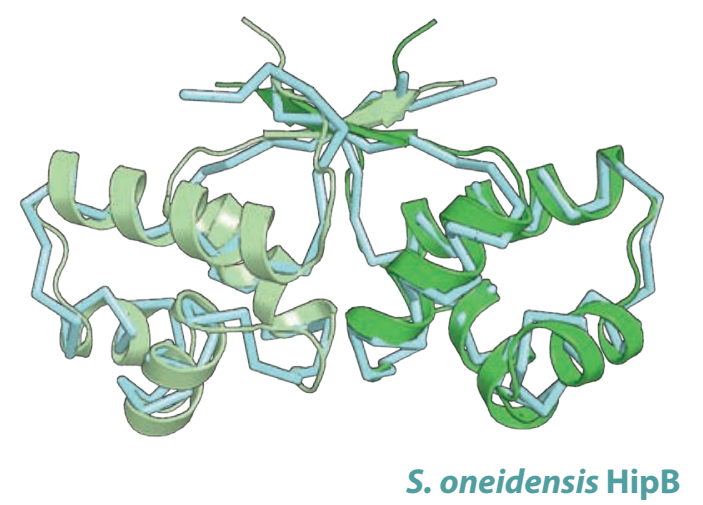

C

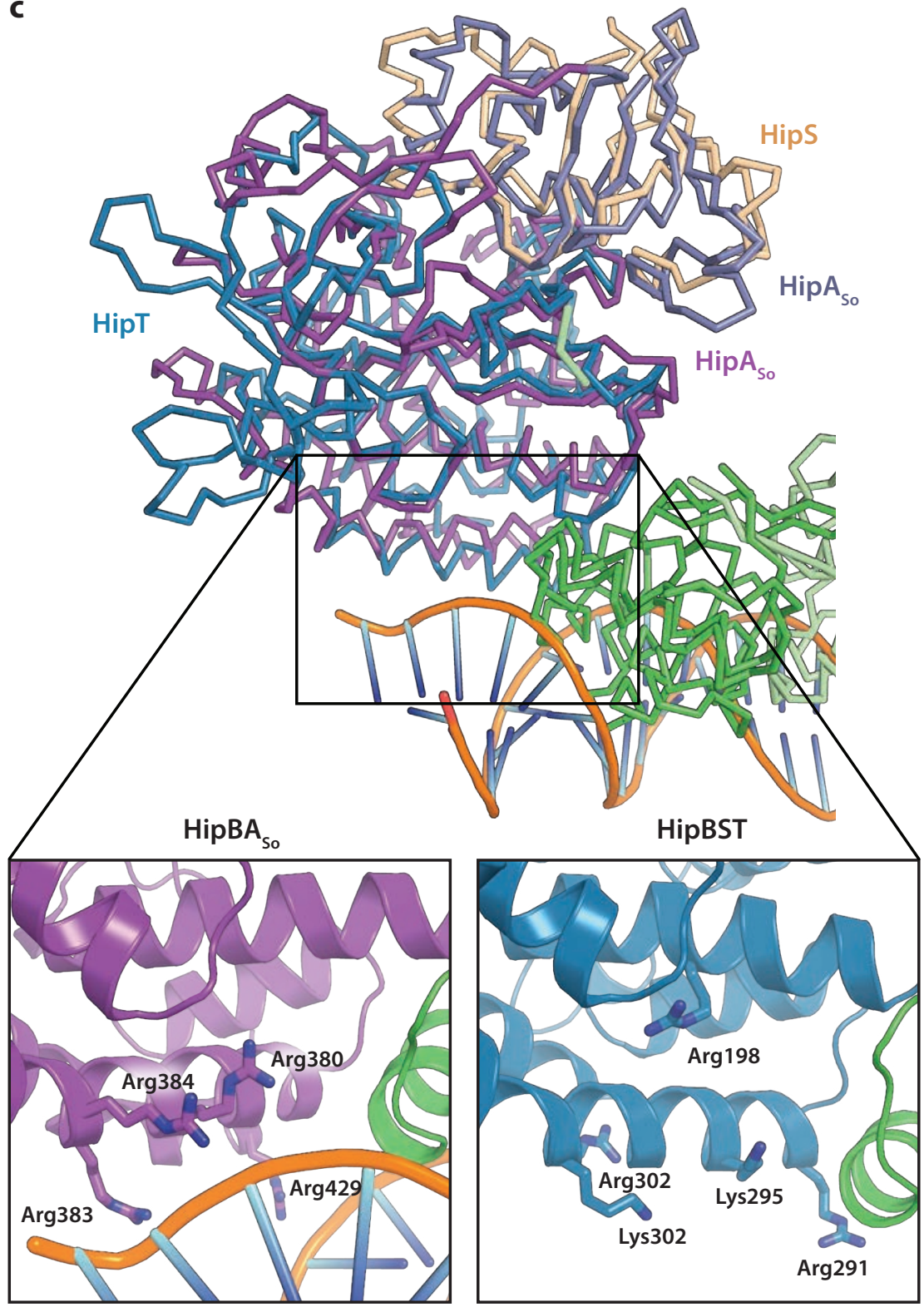

d

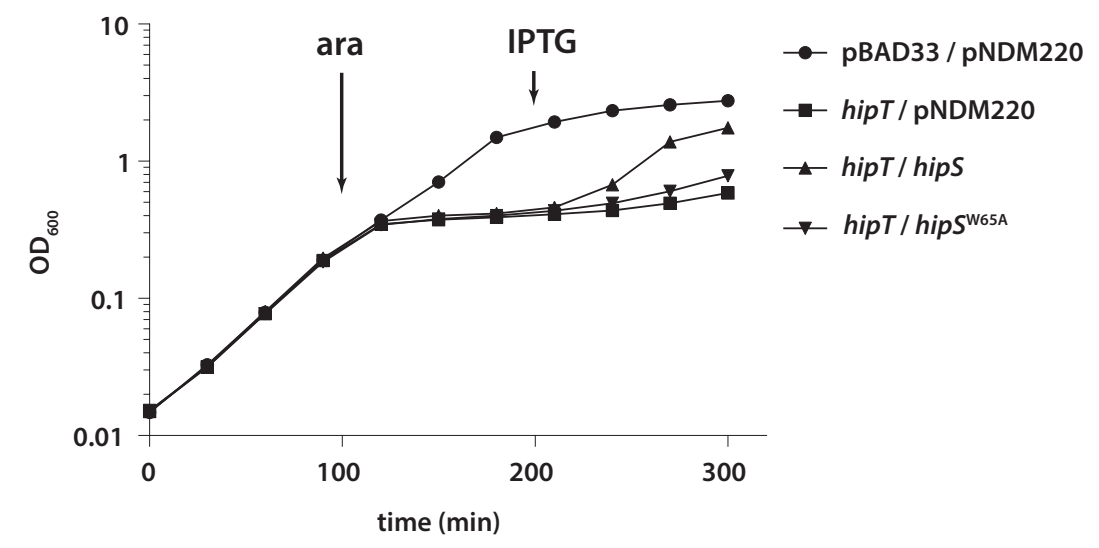


Figure S4 bioRxiv preprint doi: https://doi.org/10.1101/2022.01.28.478185; this version posted January 31, 2022. The copyright holder for this preprint (which was not certified by peer review) is the author/funder, who has granted bioRxiv a license to display the preprint in perpetuity. It is made available under aCC-BY 4.0 International license.

a

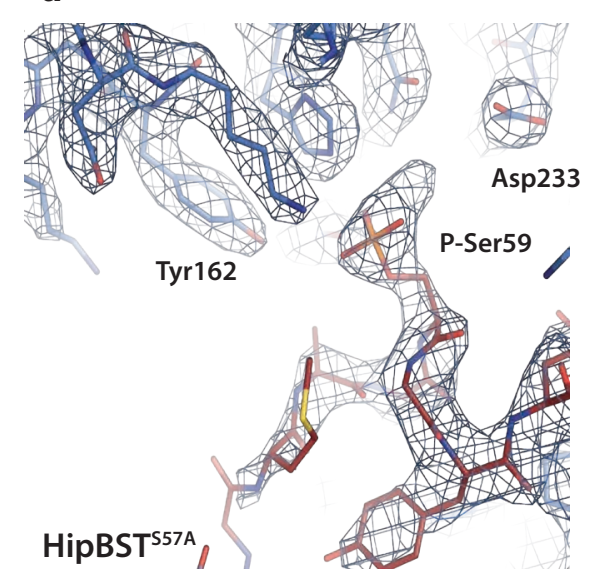

b

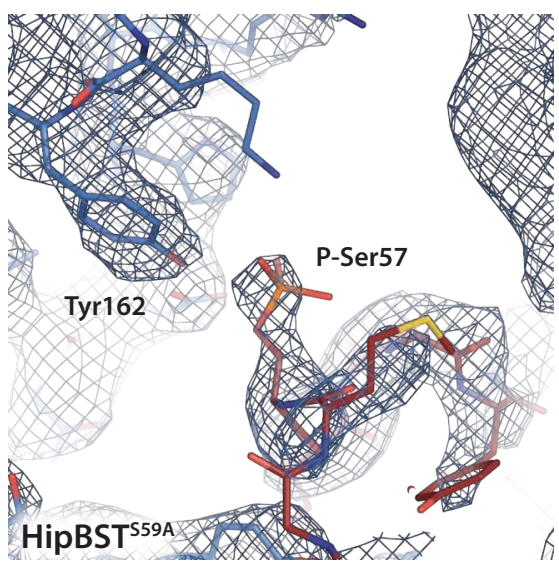

c

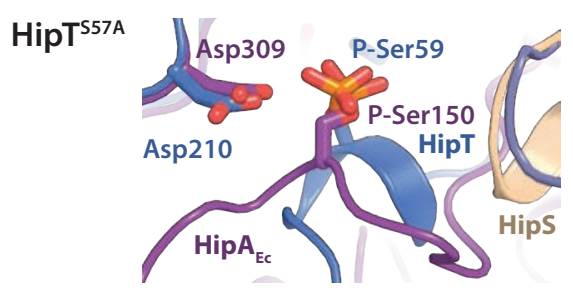

HipT $^{\mathrm{S59A}}$

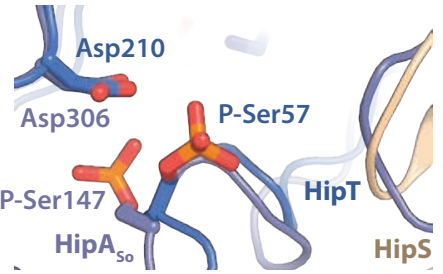

d

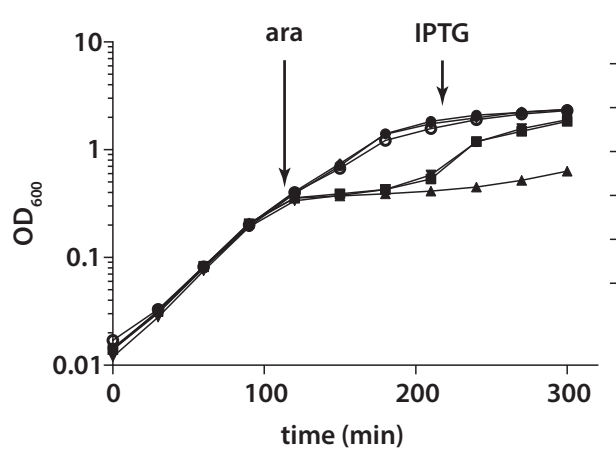

e

- pBAD33 / hipBS

- $\operatorname{hipT}(\mathrm{SIS}, w t) / \mathrm{hipBS}$

- $\operatorname{hipT}(\mathrm{DIS}) / \mathrm{hipBS}$

$\rightarrow \operatorname{hipT}(\mathrm{SID}) / \mathrm{hip} B S$

- $\operatorname{hipT}$ (AIS) / hipBS

- $\operatorname{hipT}(\mathrm{SIA}) / \mathrm{hipBS}$

f

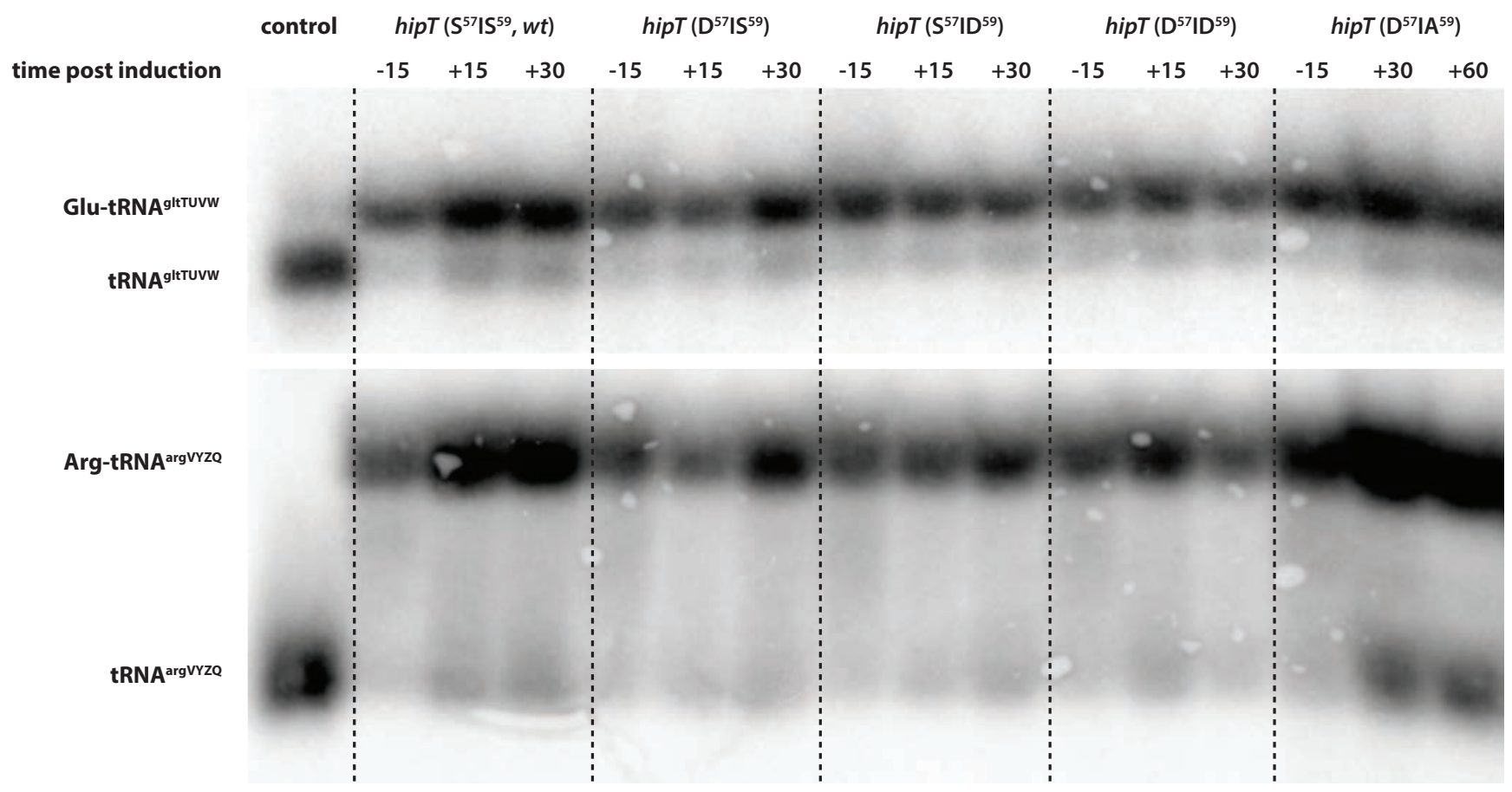

-- pBAD33 / hipBS

- $\operatorname{hipT}(\mathrm{SIS}, w t) /$ hipBS

$\leftarrow \operatorname{hipT}(\mathrm{AIA}) / \mathrm{hipBS}$

$\rightarrow \operatorname{hipT}(\mathrm{DIA}) / \mathrm{hipBS}$

$\rightarrow \operatorname{hipT}$ (AID) / hipBS

$\rightarrow \operatorname{hipT}$ (DID) / hipBS 
Figure S5 bioRxiv preprint doi: https://doi.org/10.1101/2022.01.28.478185; this version posted January 31, 2022. The copyright holder for this preprint (which was not certified by peer review) is the author/funder, who has granted bioRxiv a license to display the preprint in perpetuity. It is made available under aCC-BY 4.0 International license.

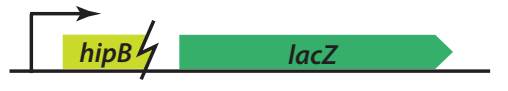

glucose

(represses hipBST)

PBAD33

hipBST ${ }^{\mathrm{D} 2330}$

hipBS

hipBT ${ }^{\mathrm{D} 2330}$

hipST ${ }^{\mathrm{D} 2330}$

hipB

hips

hipT $^{\mathrm{D} 2330}$

C

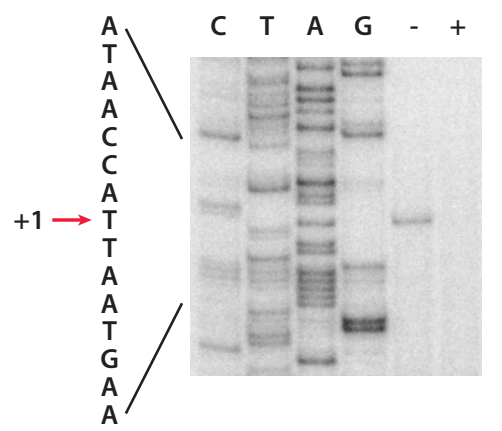

b

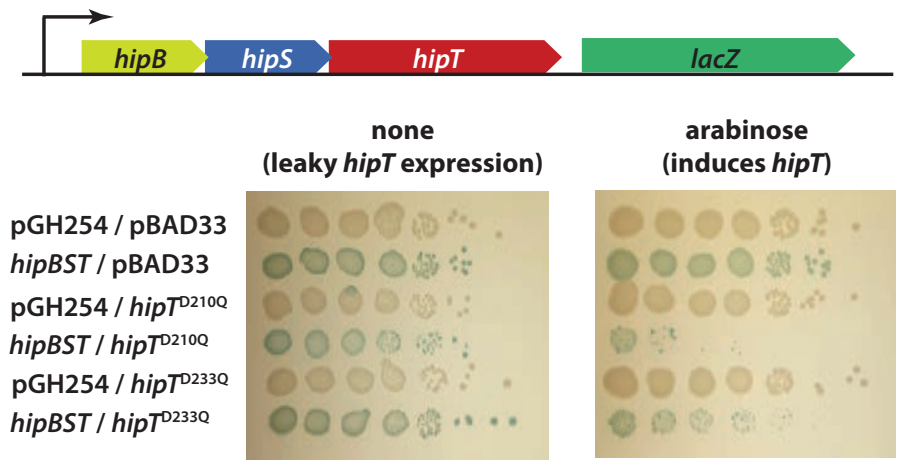

e

$-35$

arabinose (induces hipBST)

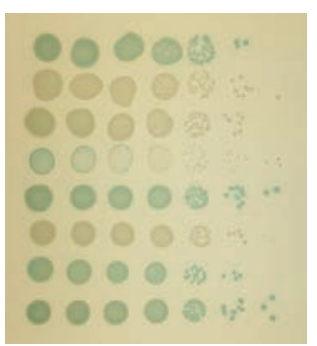

d

time post

induction

$\min -23060$ C T A G

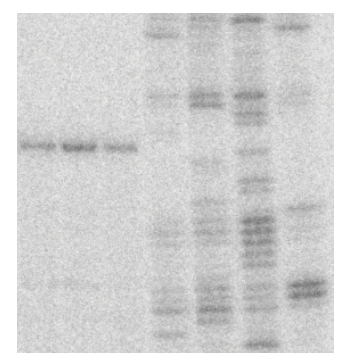

CCTTTTTCTTTGCCAATGAGTTATATGAGCTAAATTATAACCATTAATGAAAAATGGTTCATTTGTGAT

\section{ACTTAACGAATTTATCTGACCATAAATGATCTTGTTTTTGTCTTTTATCAATCGAAAGGTTCTTTAATG}

SD

Met

f

pBAD33 / hipB

hipt $\left(S^{57} \mid S^{59}, w t\right) / h i p B$

hipT $\left(D^{57} \mid S^{59}\right) /$ hipB

hipT $\left(\mathrm{S}^{57} \mathrm{D}^{59}\right) / \mathrm{hipB}$

hipt $\left(\mathrm{A}^{57} \mathrm{I}^{59}\right) /$ hipB

hipt $\left(\mathrm{S}^{57} \mid \mathrm{A}^{59}\right) /$ hipB

hipT $\left(A^{57} I^{59}\right) /$ hipB

hipT $\left(D^{57} \mid A^{59}\right) / h i p B$

hipT $\left(\mathrm{A}^{57} \mathrm{D}^{59}\right) / \mathrm{hipB}$

hipT $\left(D^{57} D^{59}\right) /$ hipB
hipT ${ }^{\mathrm{D} 2100}+$ hipB

\begin{tabular}{|c|c|c|}
\hline $\begin{array}{c}\text { glucose } \\
\text { (represses hipT) }\end{array}$ & $\begin{array}{c}\text { arabinose } \\
\text { (induces hipT) }\end{array}$ & $\begin{array}{l}\text { arabinose + IPTG } \\
\text { (induces hipB) }\end{array}$ \\
\hline 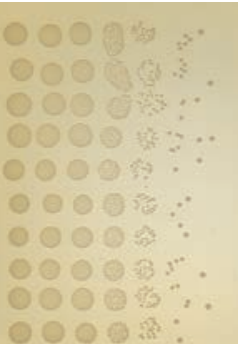 & 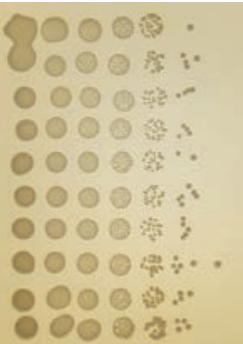 & 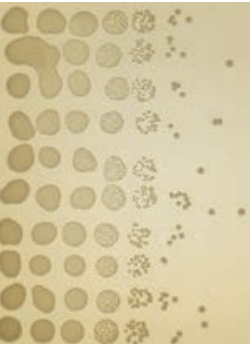 \\
\hline
\end{tabular}


Figure S6 bioRxiv preprint doi: https://doi.org/10.1101/2022.01.28.478185; this version posted January 31, 2022. The copyright holder for this preprint (which was not certified by peer review) is the author/funder, who has granted bioRxiv a license to display the preprint in perpetuity. It is made available under aCC-BY 4.0 International license.

a

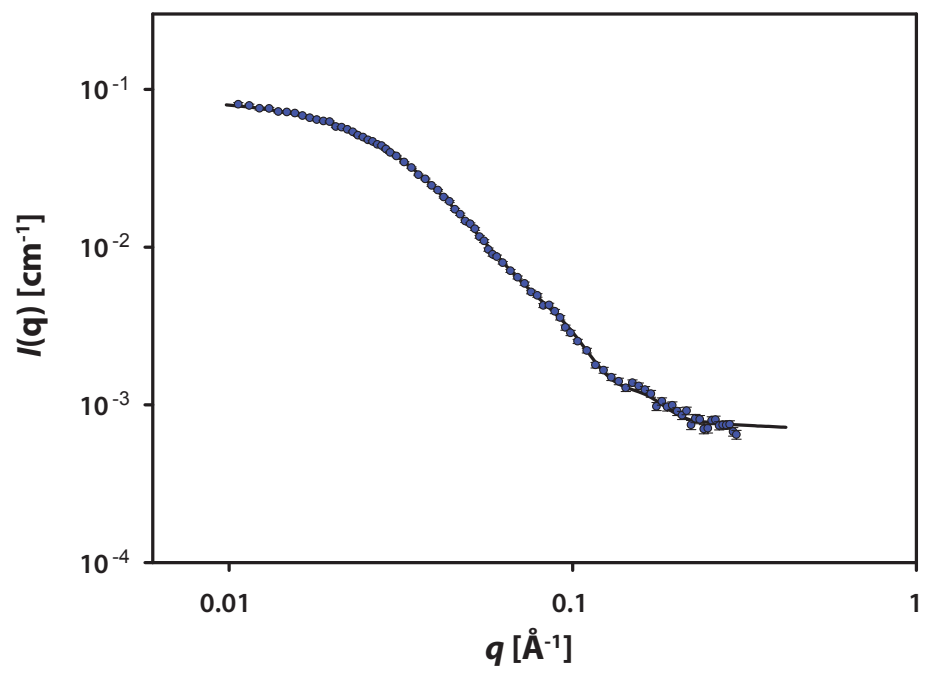

C

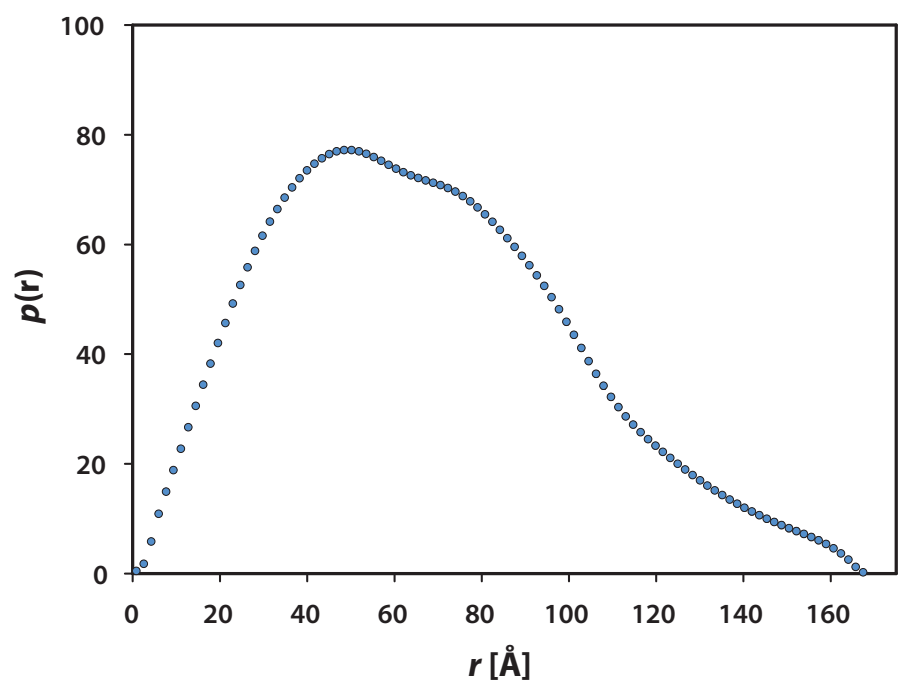

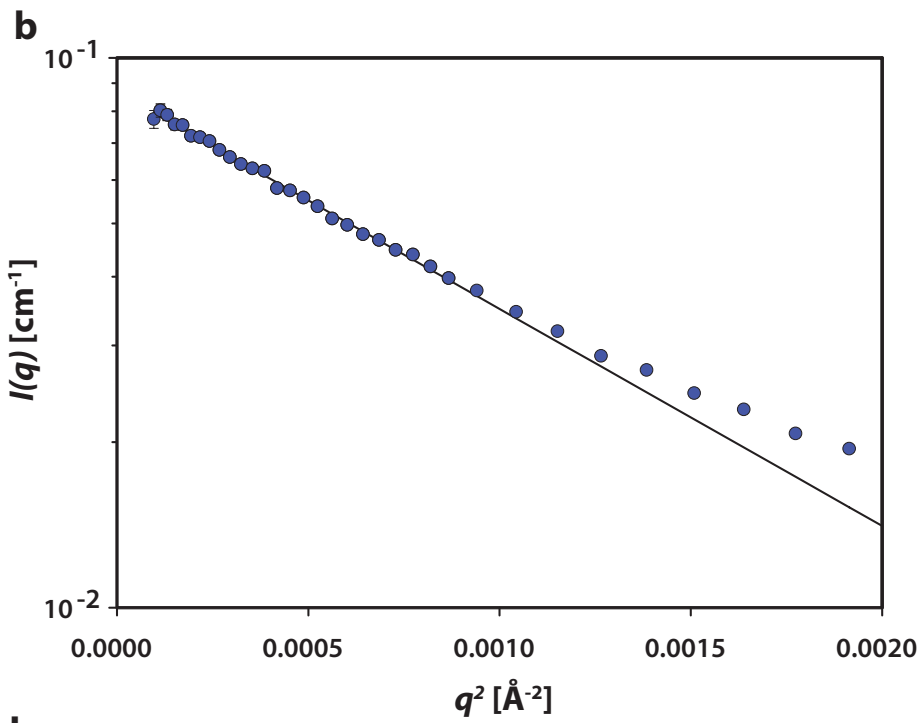

d

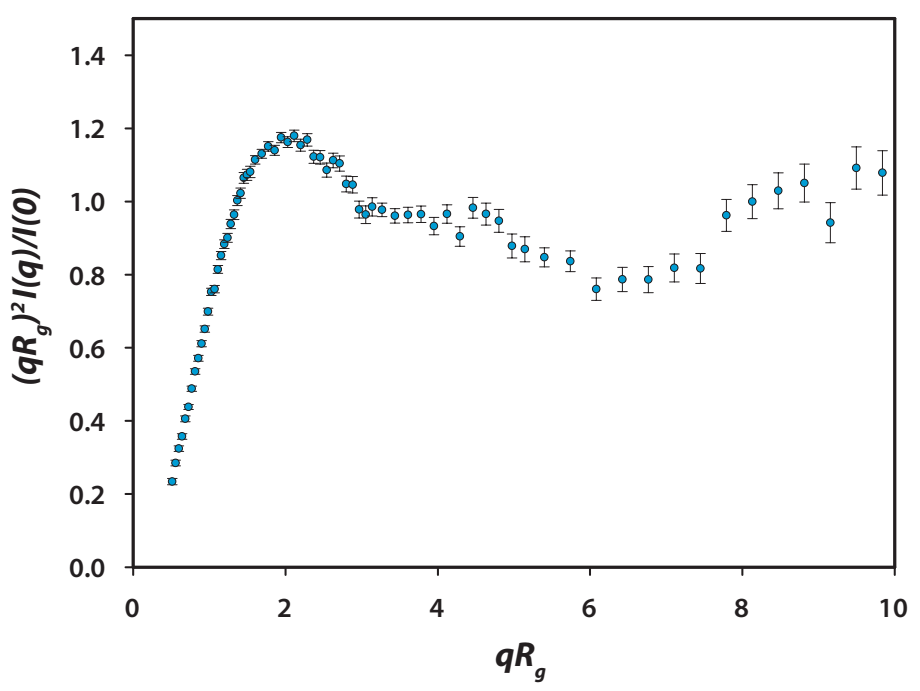


Figure S7 bioRxiv preprint doi: https://doi.org/10.1101/2022.01.28.478185; this version posted January 31, 2022. The copyright holder for this preprint (which was not certified by peer review) is the author/funder, who has granted bioRxiv a license to display the preprint in perpetuity. It is made available under aCC-BY 4.0 International license.

a

\begin{tabular}{l|ll|} 
HipT & N-subdomain 2 & Core kinase \\
\hline CDK5 & N-terminal lobe & C-terminal lobe \\
\cline { 2 - 3 }
\end{tabular}
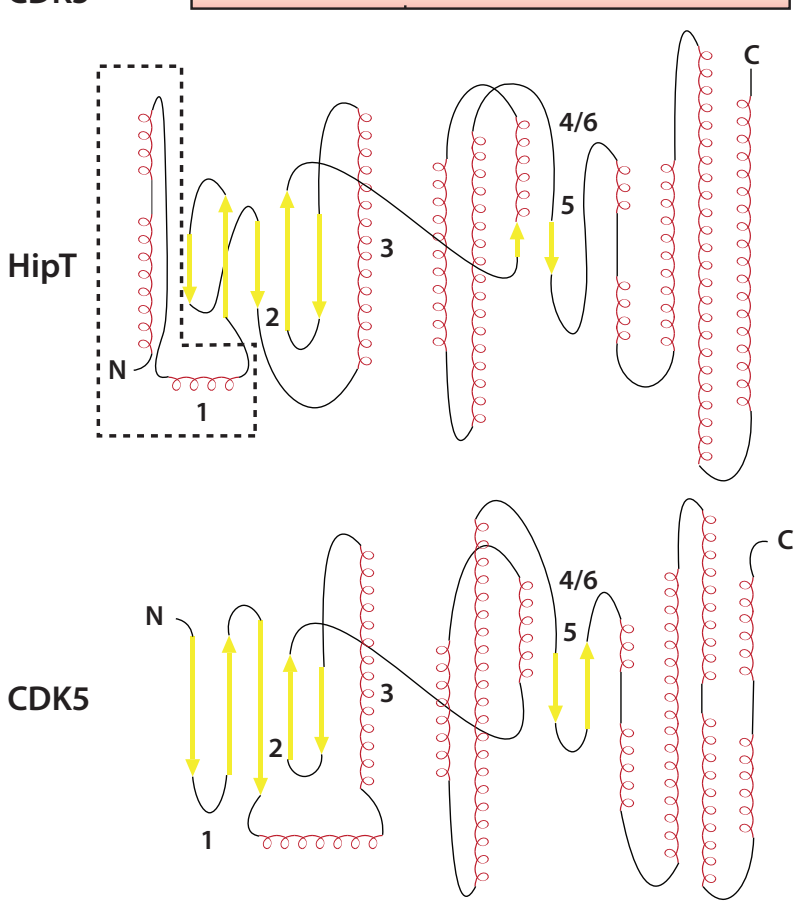

\begin{tabular}{lll}
\hline 1: Gly-rich motif & 3: Glu99/Glu51 & 5: Asn215/Asn131 \\
2: Lys86/Lys33 & 4: Asp210/Asp126 & 6: Asp233/Asp144
\end{tabular}

c

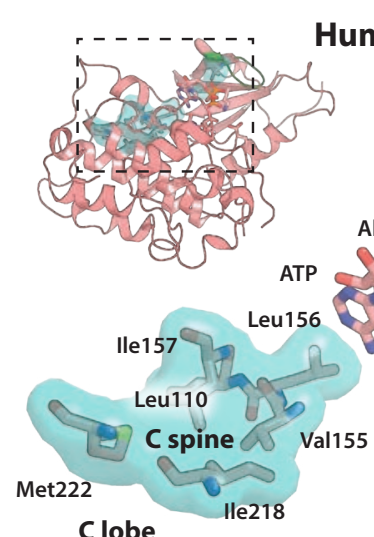

Human CDK9

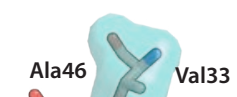
Val33
N lobe

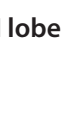

d

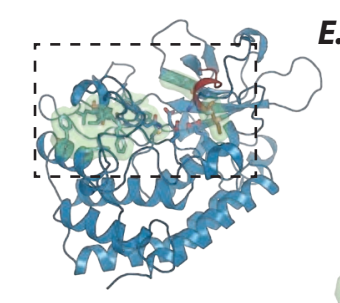

E. coli 0127:H6 HipT

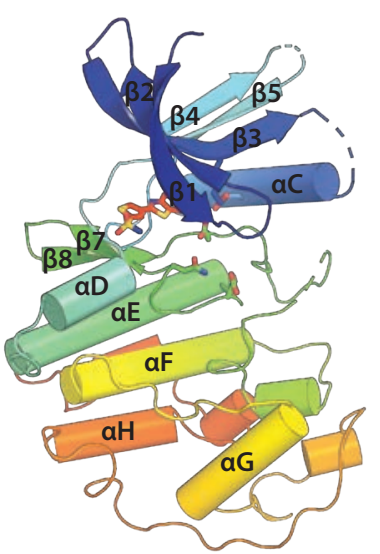

Human Cdk5

Human Cdk5
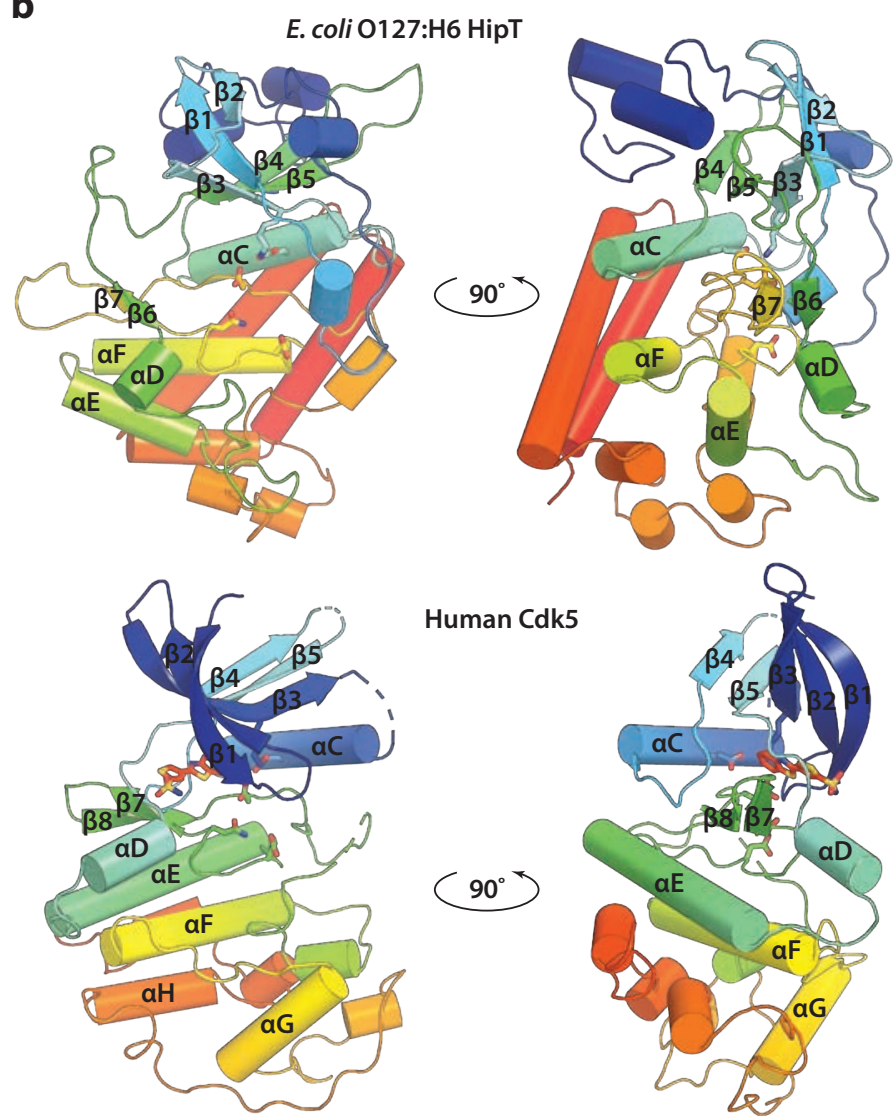

(90)

e

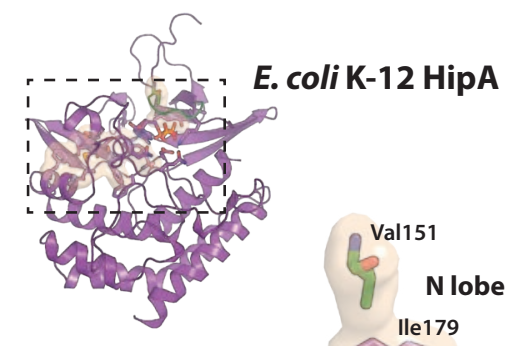

N lobe

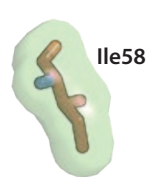

Clobe
Clobe

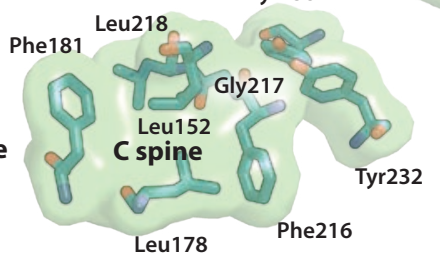

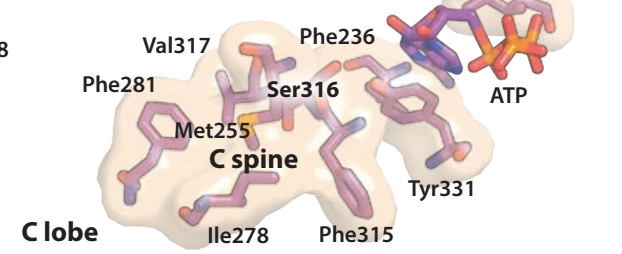

